\title{
Translation Invariant Operators
}

\author{
Alejandro Molero Casanova \\ Advisor: F. Javier Soria de Diego
}

Master in Advanced Mathematics

University of Barcelona

Barcelona, June 2018 



\section{Contents}

$\begin{array}{ll}\text { Introduction } & 1\end{array}$

1 Preliminary definitions and results 3

$1.1 \quad L^{p}$ spaces and bounded operators . . . . . . . . . . . . . . 3

1.2 Vector valued $L^{p}$ spaces . . . . . . . . . . . . . . . . . 5

1.3 Convolution and Fourier transform . . . . . . . . . . . . . . 7

1.4 Rapidly decreasing functions and tempered distributions . . . . . . 10

1.5 Sobolev spaces . . . . . . . . . . . . . . . . . . . 12

2 Multipliers $\quad 13$

2.1 First definitions . . . . . . . . . . . . . . . . . . . 13

2.2 The spaces $L_{p}^{q}$ and $M_{p}^{q} \ldots \ldots \ldots \ldots \ldots \ldots$

2.3 Properties of $L_{p}^{q}$ and $M_{p}^{q} \ldots \ldots \ldots \ldots \ldots \ldots$

3 Littlewood-Paley theory 33

3.1 Introduction . . . . . . . . . . . . . . . . . 33

3.2 Littlewood-Paley operators and orthogonal decomposition . . . . . 37

3.3 Orthogonal decomposition in dyadic sets . . . . . . . . . . . . . . . . 44

4 Multipliers theorems $\quad 51$

4.1 Marcinkiewicz's theorem . . . . . . . . . . . . . . . . 51

4.2 Mihlin-Hörmander's theorem . . . . . . . . . . . . . . . . 60

$\begin{array}{lll}5 & \text { The ball multiplier } & 67\end{array}$

5.1 Perron's tree . . . . . . . . . . . . . . . . . . . . . 68

5.2 Boundedness on half-planes . . . . . . . . . . . . . . 73

5.3 The answer to the ball multiplier problem . . . . . . . . . . . . 77

$\begin{array}{lr}\text { Bibliography } & 79\end{array}$

$\begin{array}{lr}\text { Index } & 80\end{array}$ 



\section{Acknowledgments}

I would like to express my most sincere gratitude to my advisor, Professor F. Javier Soria de Diego, both for proposing me such an interesting topic for my thesis, and for helping and guiding me thoroughly during the whole development of the project.

I would also like to thank my friends and family for their support and affection, specially during my weakest moments. To my mathematician friends, thanks as well for letting me share my work with you and for your thoughts on it. 



\section{Introduction}

The theory of translation invariant operators is a branch that involves techniques and concepts from a wide variety of fields such as functional analysis, harmonic analysis or Fourier analysis, and whose main goal is to find and describe boundedness conditions and norm estimates on such objects. Its study started to firmly develop around the second half of the last century, thanks to the work of Hörmander, Fefferman, Jodeit, Stein, Marcinkiewicz, and so many others, but it is still a subject of great interest nowadays, not only because of its applications and relations to other fields, such as partial differential equations or summability methods for Fourier coefficients, but also because of the large amount of open questions that the theory still has to answer. For instance, it is not known yet a complete characterization on when such an operator is bounded between two Lebesgue spaces. Maybe that is one of its most surprising aspects: even though its main object of study is a class of operators which are initially defined with a very simple property (namely, commuting with translations), it turns out that this condition gives rise to a rich structure that requires of much more sophisticated tools in order to understand it.

The current development of the theory tries to follow that path, using those tools, such as the theory of distributions and Fourier transform, to translate the problem of boundedness of operators to a problem of studying properties of certain functions, which in this context are called multipliers. This is indeed a helpful step, for one can then find symmetry properties on the classes of multipliers of bounded operators between two Lebesgue spaces, and even arrive to the conclusion that when both spaces are actually the same, every multiplier is a bounded function.

Yet, starting from a bounded function, finding which of its properties (analytical, geometrical,...) might be relevant in order to have a multiplier is a tough task that, as we say, is still barely solved. It is known, for instance, that when the multiplier is a characteristic function of a set, the geometry of the set plays a huge role. The maximum exponent of this fact is probably the, now disproven, multiplier problem for the ball. Even when the geometry of the set gives good boundedness properties (such as the case of a polygon), there is still much work to do on finding sharp constants for the norm of such operators. On the other hand, regularity conditions seem to affect as well, thanks to a couple of results due to Hörmander and Marcinkiewicz. 
Our goal in this Master's Thesis is to give an overview of the most relevant aspects of the theory, starting from the most basic definitions, and ending precisely in the study of Hörmander's and Marcinkiewicz's results and the multiplier problem for the ball. Chapters 1 and 2 cover the initial tools that one needs in order to attack this topic, such as the essential elements of the theory of function spaces and distributions, and the definition of translation invariant operators, together with their identification with multipliers and the properties of multipliers' classes, given mostly by Theorem 2.15. We then move to Chapter 3 in order to review a theory due to Littlewood and Paley about decomposition of functions in the frequency domain using multipliers, which gives estimates on such decompositions in terms of the original function. The idea behind this theory is to extend estimates which are natural on the space of square integrable functions to other spaces of integrable functions, as done in Theorem 3.4. This theory is later used in Chapter 4 to prove the mentioned results of Hörmander and Marcinkiewicz (Theorems 4.2 and 4.5), and we see some applications of these results to multipliers with simple homogeneity properties. Finally, we focus on Chapter 5 to review this influence of geometrical properties on boundedness of this class of operators, where we have exposed Fefferman's work on the ball multiplier problem. This work, which culminates with the conclusion of Theorem 5.6, represents a nice example on how the theory of translation invariant operators intersects with other seemingly unrelated branches, for it uses a geometrical construction originated around the first half of the past century known as Perron's tree, which was originally proposed for solving Kakeya's needle problem. 


\section{Chapter 1}

\section{Preliminary definitions and results}

In this first chapter, we review some of the basic theory that we might need in order to proceed properly with the actual topic of the thesis. In particular, we give some well known definitions and results regarding function spaces (such as the Lebesgue spaces, the Schwarz class, or Sobolev spaces) and operators between those spaces. We do so essentially for completeness of the work and for fixing the notation that we will use later on throughout the whole manuscript.

\section{1 $\quad L^{p}$ spaces and bounded operators}

Definition 1.1. Given $1 \leq p<\infty$, we will denote by $L^{p}$ the Lebesgue space of Lebesgue measurable functions $f: \mathbb{R}^{n} \rightarrow \mathbb{R}$ such that

$$
\|f\|_{p}=\left(\int_{\mathbb{R}^{n}}|f(x)|^{p} d x\right)^{1 / p}
$$

is finite, together with the norm $\|\cdot\|_{p}$. For $p=\infty, L^{\infty}$ will be the space of Lebesgue measurable functions $f: \mathbb{R}^{n} \rightarrow \mathbb{R}$ which are bounded, and the norm in this case is given by

$$
\|f\|_{\infty}=\sup _{x \in \mathbb{R}^{n}}|f(x)|
$$

We will explicitly write $L^{p}\left(\mathbb{R}^{n}\right)$ when we need to emphasize the dimension $n$ of the euclidean space. Recall that, with these norms, $L^{p}$ is a Banach space for $1 \leq p \leq \infty$, and it is actually a Hilbert space for $p=2$. When $p=\infty$, we will also denote as $L_{0}^{\infty}$ the subspace of $L^{\infty}$ of functions vanishing at infinity.

Definition 1.2. Given two Banach spaces $X, Y$, a linear operator $A$ from $X$ to $Y$ is bounded if there exists some constant $C>0$ such that $\|A f\|_{Y} \leq C\|f\|_{X}$, for all $f \in X$.

Boundedness estimates as the former will also be denoted as $\|A f\|_{Y} \lesssim\|f\|_{X}$, specially when the constant $C$ is not relevant. When $X=L^{p}$ and $Y=L^{q}$ for some $1 \leq p, q, \leq \infty$, a bounded operator $A$ is also called strong type $(p, q)$. In this setting, 
we will sometimes write $A: L^{p} \rightarrow L^{q}$ to denote that $A$ is bounded from $L^{p}$ t o $L^{q}$. Recall that the space $\mathcal{L}\left(L^{p}, L^{q}\right)$ of bounded and linear operators from $L^{p}$ to $L^{q}$ is a Banach space with the norm

$$
\|A\|=\sup _{f \in L^{p}} \frac{\|A f\|_{q}}{\|f\|_{p}}
$$

for $L^{q}$ is a Banach space [1, Theorem 2.29]. We will usually avoid writing sub indices for the operator norm unless they are necessary (for instance, when comparing the norm of the operator acting between different $L^{p}$ spaces).

When $A$ is a linear operator, boundedness and continuity are equivalent concepts. We can think, though, about another type of continuity, called continuity in measure, which is weaker than the former, but sometimes easier to see, and sufficient for certain applications.

Definition 1.3. For $1 \leq p<\infty$, the weak $L^{p}$ space, denoted as $L^{p, \infty}$, is the set of Lebesgue measurable functions such that

$$
\|f\|_{p, \infty}:=\sup _{t>0} t\left|\left\{x \in \mathbb{R}^{n}:|f(x)|>t\right\}\right|^{1 / p}
$$

is finite.

It is easy to see that $L^{p} \subset L^{p, \infty}$ for any $1 \leq p<\infty$, and it holds that $\|f\|_{p, \infty} \leq\|f\|_{p}$. In fact,

$$
\begin{aligned}
t\left|\left\{x \in \mathbb{R}^{n}:|f(x)|>t\right\}\right|^{1 / p} & =\left(\int_{\left\{x \in \mathbb{R}^{n}:|f(x)|>t\right\}} t^{p} d x\right)^{1 / p} \\
& <\left(\int_{\left\{x \in \mathbb{R}^{n}:|f(x)|>t\right\}}|f(x)|^{p} d x\right)^{1 / p} \\
& \leq\left(\int_{\mathbb{R}^{n}}|f(x)|^{p} d x\right)^{1 / p}=\|f\|_{p},
\end{aligned}
$$

and so $\|f\|_{p, \infty}^{p} \leq\|f\|_{p}^{p}$.

Definition 1.4. An operator $A$ from $L^{p}$ to $L^{q, \infty}$ is called weak type $(p, q)$ if there exists some constant $C>0$ such that $\|A f\|_{q, \infty} \leq C\|f\|_{p}$.

There exists some duality correspondence between the $L^{p}$ spaces. Recall that given a real Banach space $X$, its dual space $X^{\prime}$ is the space of linear and bounded maps from $X$ to $\mathbb{R}$. The elements of $X^{\prime}$ are usually called functionals. It turns out that, when $X=L^{p}$ for $1 \leq p<\infty$, there is a very natural way to construct functionals on $L^{p}$. Denote as $p^{\prime}$ the conjugate exponent of $p$; that is, $p^{\prime}$ is such that

$$
\frac{1}{p}+\frac{1}{p^{\prime}}=1
$$


Now, take $g \in L^{p^{\prime}}$, and consider the map

$$
u_{g}(f)=\int_{\mathbb{R}^{n}} f(x) g(x) d x .
$$

Then, using Hölder's inequality,

$$
\left|u_{g}(f)\right| \leq\|f g\|_{1} \leq\|f\|_{p}\|g\|_{p^{\prime}}
$$

and so $u_{g} \in\left(L^{p}\right)^{\prime}$. This correspondence $g \rightarrow u_{g}$ is actually an isometric isomorphism, and this gives us the duality correspondence between the $L^{p}$ spaces, which is usually referred as Riesz representation theorem for $\left(L^{p}\right)^{\prime}[1$, Theorem 4.10]:

$$
\left(L^{p}\right)^{\prime}=L^{p^{\prime}}, 1 \leq p<\infty .
$$

For $p=\infty$, the same construction works for building functionals on $L^{\infty}$, and so $L^{1} \subset\left(L^{\infty}\right)^{\prime}$. The equality, though, is no longer true.

We point out now an extrapolation result that we will need in the future. It deals with proving boundedness of certain types of operators, regarding that we know that their are bounded in some $L^{p}$ space. The proof can be found in [5, Theorem 5.323], using Calderón-Zygmund decomposition.

Theorem 1.5. Let $K$ be a function on $\mathbb{R}^{n} \backslash\{0\}$ such that

$$
\sup _{R>0} \int_{R<|x|<2 R}|K(x)| d x=A_{1}<\infty
$$

and

$$
\sup _{y \neq 0} \int_{|x| \geq 2|y|}|K(x-y)-K(x)| d x=A_{2} .
$$

Also, suppose that $K$ is given as a distribution by

$$
K(\phi)=\lim _{\delta \rightarrow 0} \int_{|x| \geq \delta} K(x) \phi(x) d x .
$$

Finally, denote by $T$ the operator given by the convolution with $K$, and assume that $T$ is strong type $(r, r)$ for some $1 \leq r \leq \infty$. Then, $T$ is also bounded from $L^{p}$ to $L^{p}$ for $1<p<\infty$, and it is weak type $(1,1)$.

\section{$1.2 \quad$ Vector valued $L^{p}$ spaces}

Definition 1.6. Let $\left(X,\|\cdot\|_{X}\right)$ be a Banach space. Given $1 \leq p<\infty$, we denote by $L^{p}\left(\mathbb{R}^{n}, X\right)$ the space of functions $f: \mathbb{R}^{n} \rightarrow X$ such that

$$
\|f\|_{p, X}:=\left(\int_{\mathbb{R}^{n}}\|f(x)\|_{X}^{p} d x\right)^{1 / p}
$$

is finite. For $p=\infty, L^{\infty}\left(\mathbb{R}^{n}, X\right)$ is the space of functions $f: \mathbb{R}^{n} \rightarrow X$ such that

$$
\|f\|_{\infty, X}:=\sup _{x \in \mathbb{R}^{n}}\|f(x)\|_{X}
$$

is finite. 
Definition 1.7. Let $\left(X,\|\cdot\|_{X}\right)$ be a Banach space. Given $1 \leq p<\infty$, we denote by $L^{p, \infty}\left(\mathbb{R}^{n}, X\right)$ the space of functions $f: \mathbb{R}^{n} \rightarrow X$ such that

$$
\|f\|_{p, \infty, X}:=\sup _{t>0} t\left|\left\{x \in \mathbb{R}^{n}:\|f(x)\|_{X}>t\right\}\right|^{1 / p}
$$

is finite.

The spaces $L^{p}\left(\mathbb{R}^{n}, X\right)$ and $L^{p, \infty}\left(\mathbb{R}^{n}, X\right)$ are a natural generalization of the $L^{p}$ and $L^{p, \infty}$ spaces, when the functions are not necessarily real valued.

Example 1.8. Given $1 \leq r<\infty$, denote by $\ell^{r}$ the space of real sequences $\left\{a_{k}\right\}_{k \in \mathbb{Z}}$ such that

$$
\left\|\left\{a_{k}\right\}_{k \in \mathbb{Z}}\right\|_{\ell^{r}}:=\left(\sum_{k \in \mathbb{Z}}\left|a_{k}\right|^{r}\right)^{1 / r}<+\infty,
$$

and denote $\ell^{\infty}$ the space of real sequences such that

$$
\left\|\left\{a_{k}\right\}_{k \in \mathbb{Z}}\right\|_{\ell^{\infty}}:=\sup _{k \in \mathbb{Z}}\left|a_{k}\right|<+\infty .
$$

If $f \in L^{p}\left(\mathbb{R}^{n}, \ell^{r}\right)$, it means that $f(x)$ is a sequence in $\ell^{r}$ for each $x \in \mathbb{R}^{n}$. Thus, we might write

$$
f=\left\{f_{k}\right\}_{k \in \mathbb{Z}},
$$

where $f_{k}$ is a function for each $k \in \mathbb{Z}$, in such a way that

$$
\|f(x)\|_{\ell^{r}}=\left\|\left\{f_{k}(x)\right\}_{k \in \mathbb{Z}}\right\|_{\ell^{r}}=N(x)<+\infty,
$$

and moreover, the function $N$ of norms is a function in $L^{p}$.

We will need these spaces, as well as some results about them, in the considerations developed in Chapter 3. The first of all states that a bounded operator from $L^{p}$ to $L^{q}$ can be extended in a very natural way to an operator from $L^{p}\left(\mathbb{R}^{n}, \ell^{2}\right)$ to $L^{q}\left(\mathbb{R}^{n}, \ell^{2}\right)$, which turns out to be bounded. A proof of this result can be found in [5, Theorem 5.5.1].

Theorem 1.9. Let $T$ be a bounded linear operator from $L^{p}$ to $L^{q}$. Then, the operator

$$
\vec{T}\left(\left\{f_{k}\right\}_{k \in \mathbb{Z}}\right)=\left\{T\left(f_{k}\right)\right\}_{k \in \mathbb{Z}},
$$

is bounded from $L^{p}\left(\mathbb{R}^{n}, \ell^{2}\right)$ to $L^{q}\left(\mathbb{R}^{n}, \ell^{2}\right)$; that is,

$$
\left\|\left(\sum_{k \in \mathbb{Z}}\left|T\left(f_{k}\right)\right|^{2}\right)^{1 / 2}\right\|_{q} \leq C\left\|\left(\sum_{k \in \mathbb{Z}}\left|f_{k}\right|^{2}\right)^{1 / 2}\right\|_{p}
$$

and we actually have that $C=C(p, q,\|T\|)$. 
The second theorem is a generalization of the extrapolation theorem 1.5; that is, given two Banach spaces $X, Y$, and a certain operator $\vec{T}$ bounded from $L^{r}\left(\mathbb{R}^{n}, X\right)$ to $L^{r}\left(\mathbb{R}^{n}, Y\right)$, it gives conditions for $\vec{T}$ so that it is actually bounded from $L^{p}\left(\mathbb{R}^{n}, X\right)$ to $L^{p}\left(\mathbb{R}^{n}, Y\right)$ for several values of $p$. A proof can be found in [5, Theorem 5.6.1], using Calderón-Zygmund decomposition.

Theorem 1.10. Let $X, Y$ be Banach spaces. Let $\vec{K}: \mathbb{R}^{n} \backslash\{0\} \rightarrow L(X, Y)$ be a map such that for some $A>0$ and $\vec{K}_{0} \in L(X, Y)$ we have:

i) $\|\vec{K}(x)\|_{X \rightarrow Y} \leq A|x|^{-n}$, where $\|\cdot\|_{X \rightarrow Y}$ denotes the operator norm in $L(X, Y)$.

ii) $\sup _{y \in \mathbb{R}^{n} \backslash\{0\}} \int_{|x| \geq 2|y|}\|\vec{K}(x-y)-\vec{K}(x)\|_{X \rightarrow Y} d x \leq A$.

iii) $\lim _{\varepsilon \rightarrow 0}\left\|\int_{\varepsilon \leq|y| \leq 1} \vec{K}(y) d y-\vec{K}_{0}\right\|_{X \rightarrow Y}=0$.

Moreover, let $\vec{T}$ be an operator given by

$$
\vec{T}(f)(x)=\lim _{\varepsilon \rightarrow 0} \int_{\varepsilon \leq|y|} \vec{K}(y)(f(x-y)) d y,
$$

for any function $f$ taking values in $X$, and assume that $\vec{T}$ is bounded from $L^{r}\left(\mathbb{R}^{n}, X\right)$ to $L^{r}\left(\mathbb{R}^{n}, Y\right)$ for some $1<r \leq \infty$ with norm $B$. Then, there exist $C_{n}, C_{n}^{\prime}>0$ such that $\vec{T}$ is bounded from $L^{p}\left(\mathbb{R}^{n}, X\right)$ to $L^{p}\left(\mathbb{R}^{n}, Y\right)$ for all $1<p<\infty$ with

$$
\|\vec{T}(f)\|_{p, Y} \leq C_{n} \max \left(p,(p-1)^{-1}\right)(A+B)\|f\|_{p, X},
$$

and it is also weak type $(1,1)$ with

$$
\|\vec{T}(f)\|_{1, \infty, Y} \leq C_{n}^{\prime}(A+B)\|f\|_{1, X} .
$$

\subsection{Convolution and Fourier transform}

We give now a couple of concepts related with $L^{1}$ functions. These will be extremely useful in the following chapters, for most of the theory we are going to develop is based on them. The first of them is the convolution of two functions.

Definition 1.11. Given $f, g \in L^{1}$, the convolution of $f$ and $g$ is the function $f * g$ defined as

$$
(f * g)(x)=\int_{\mathbb{R}^{n}} f(y) g(x-y) d y, \forall x \in \mathbb{R}^{n} .
$$

One easily checks using Fubini's theorem that $f * g$ is well defined $L^{1}$ function, since $f$ and $g$ are $L^{1}$ functions too. Indeed,

$$
\begin{aligned}
\|f * g\|_{1} & \leq \int_{\mathbb{R}^{n}} \int_{\mathbb{R}^{n}}|f(y)||g(x-y)| d y d x \\
& =\int_{\mathbb{R}^{n}}|f(y)|\left(\int_{\mathbb{R}^{n}}|g(x-y)| d x\right) d y \\
& =\|g\|_{1} \int_{\mathbb{R}^{n}}|f(y)| d y=\|f\|_{1}\|g\|_{1} .
\end{aligned}
$$


Notice that the convolution of two functions can also be defined in other settings. For instance, if $f \in L^{1}$ and $g \in L^{p}$ for $1 \leq p \leq \infty$, then $f * g$ is a well defined $L^{p}$ function with

$$
\|f * g\|_{p}=\left\|\int_{\mathbb{R}^{n}} f(y) g(\cdot-y) d y\right\|_{p} \leq \int_{\mathbb{R}^{n}}|f(y)|\|g\|_{p} d y=\|f\|_{1}\|g\|_{p},
$$

in view of Minkowski's integral inequality.

The other important tool we review now is the Fourier transform.

Definition 1.12. Let $f \in L^{1}$. The Fourier transform of $f$ is the function $\widehat{f}$ defined as

$$
\widehat{f}(\xi)=\int_{\mathbb{R}^{n}} f(x) e^{-2 \pi i \xi \cdot x} d x, \forall \xi \in \mathbb{R}^{n}
$$

We can think of the Fourier transform operator (sometimes called simply Fourier transform, with some abuse of language) as the operator $\mathcal{F}$ defined as

$$
\mathcal{F}(f)=\widehat{f}, \forall f \in L^{1} .
$$

Notice that $\mathcal{F}$ is linear. Also, since

$$
|\mathcal{F}(f)(\xi)|=|\widehat{f}(\xi)| \leq \int_{\mathbb{R}^{n}}|f(x)| d x=\|f\|_{1},
$$

we conclude that $\mathcal{F}$ is bounded from $L^{1}$ to $L^{\infty}$ with norm at most 1 . This fact is actually improved a bit by the so called Riemann-Lebesgue lemma.

Lemma 1.13 (Riemann-Lebesgue). For $f \in L^{1}$, we have that $\widehat{f}$ is continuous and $|\widehat{f}(\xi)| \rightarrow 0$ as $|\xi| \rightarrow \infty$.

That is, the Fourier transform of any integrable function is not only bounded, but also vanishes at infinity. In particular, since it is continuous and satisfies this vanishing property, it is uniformly continuous. The fact that $\widehat{f}$ is continuous is given easily in virtue of the dominated convergence theorem. We refer to [2, Chapter 1, Section 6] for a proof of the rest of the statement.

It is a matter of computation to verify that the Fourier transform satisfies the following interesting properties.

Proposition 1.14. Let $f, g \in L^{1}, h \in \mathbb{R}^{n}, \lambda>0$ and $M \in O(n)$, where $O(n)$ is the orthogonal group of dimension $n$. Denote by $\tau_{h}$ the translation operator,

$$
\tau_{h} \varphi(x)=\varphi(x-h),
$$

$\rho_{A}$ the rotation operator,

$$
\rho_{A} \varphi(x)=\varphi(A x)
$$

and $\delta_{\lambda}$ the dilation operator,

$$
\delta_{\lambda} \varphi(x)=\varphi(x / \lambda) .
$$


Then,
i) $\widehat{\tau_{x} f}=e^{-2 \pi i x \cdot \widehat{f}}$.
ii) $\widehat{e^{-2 \pi x \cdot} f}=\tau_{x} \widehat{f}$.
iii) $\widehat{\rho_{A} f}=\rho_{A} \widehat{f}$
iv) $\widehat{\delta_{\lambda} f}=\lambda^{n} \delta_{1 / \lambda} \widehat{f}$.
v) $\widehat{f * g}=\widehat{f} \widehat{g}$.

The Fourier transform has also a nice property for functions with separated variables. In this setting, it is very easy to see the following fact.

Proposition 1.15. If $f \in L^{1}$ is such that

$$
f\left(x_{1}, \ldots, x_{n}\right)=f_{1}\left(x_{1}, \ldots, x_{k}\right) f_{2}\left(x_{k+1}, \ldots, x_{n}\right),
$$

for some $k=1, \ldots, n$, then

$$
\widehat{f}(\xi)=\widehat{f}_{1}\left(\xi_{1}, \ldots, \xi_{k}\right) \widehat{f}_{2}\left(\xi_{k+1}, \ldots, \xi_{n}\right) .
$$

Although, in principle, the Fourier transform can only be defined for $L^{1}$ functions, it turns out that it actually has a better behaviour in $L^{2}$. In this space, though, the given definition of Fourier transform might not make sense for certain functions, so we have to be careful. A natural way to start this extension to $L^{2}$ is to consider the set $L^{1} \cap L^{2}$. Here, we have a remarkable result concerning the $L^{2}$ norm, known as Plancherel's theorem. See [2, Theorem 1.18] for a proof.

Theorem 1.16 (Plancherel's theorem). For $f \in L^{1} \cap L^{2}$,

$$
\|f\|_{2}=\|\widehat{f}\|_{2} .
$$

We have that the set $L^{1} \cap L^{2}$ is dense in $L^{2}$, because $L^{1} \cap L^{2} \subset L^{2}$, and if $f \in L^{2}$ and $\varepsilon>0$, there exists a simple function $s$ (which is clearly in $L^{1} \cap L^{2}$ ) such that $\|f-s\|_{2}<\varepsilon$. Since $\mathcal{F}$ is an isometry in $L^{1} \cap L^{2}$, there exists a unique linear and bounded extension of $\mathcal{F}$ to $L^{2}$, which is an isometry as well, given by

$$
\widehat{f}=L^{2}-\lim _{n \rightarrow \infty} \widehat{f}_{n},
$$

where $\left(f_{n}\right)_{n} \subset L^{1} \cap L^{2}$ is such that $f_{n} \rightarrow f$ in $L^{2}$ as $n \rightarrow \infty$.

There are ways as well for defining the Fourier transform for $1<p<\infty$ and $p \neq 2$. When $p>2$, we must rely on the language of distributions, for there are functions on $L^{p}$ which do not admit another function as its Fourier transform. On the other hand, for $1<p<2$, we can proceed differently. First, notice that any function $f \in L^{p}$ can be expressed as $f=f_{1}+f_{2}$, where $f_{1} \in L^{1}$ and $f_{2} \in L^{2}$, because 
we could simply take $f_{1}=f \chi_{\{|f|>1\}}$, and $f_{2}=f-f_{1}$. Therefore, one has that the Fourier transform of $f$ is just

$$
\widehat{f}=\widehat{f_{1}}+\widehat{f}_{2}
$$

where $\widehat{f}_{1} \in L^{\infty}$ and $\widehat{f}_{2} \in L^{2}$. The nice thing about this procedure is that we can get an estimate on the norm of $\widehat{f}$ thanks to Riesz-Thorin convexity theorem [5, Theorem 1.3.4]. This is called Hausdorff-Young's inequality (see [2, Corollary 1.20]).

Theorem 1.17 (Hausdorff-Young's inequality). For $f \in L^{p}$ with $1<p<2$, we have that $\widehat{f} \in L^{p^{\prime}}$ and

$$
\|\widehat{f}\|_{p^{\prime}} \leq\|f\|_{p}
$$

\subsection{Rapidly decreasing functions and tempered distributions}

Denote as $C^{\infty}$ the set of all infinitely differentiable functions $f: \mathbb{R}^{n} \rightarrow \mathbb{R}$. For a function $f \in C^{\infty}$, and $\alpha=\left(\alpha_{1}, \ldots, \alpha_{n}\right) \in \mathbb{N}^{n}$, we denote

$$
D^{\alpha} f=\frac{\partial^{\alpha_{1}}}{\partial x_{1}^{\alpha_{1}}} \cdots \frac{\partial^{\alpha_{n}}}{\partial x_{n}^{\alpha_{n}}} f=\frac{\partial^{|\alpha|} f}{\partial x_{1}^{\alpha_{1}} \cdots \partial x_{n}^{\alpha_{n}}}
$$

where $|\alpha|=\alpha_{1}+\cdots+\alpha_{n}$.

Definition 1.18. A function $f \in C^{\infty}$ is rapidly decreasing if

$$
p_{k}(f)=\sup _{|\alpha| \leq k} \sup _{x \in \mathbb{R}^{n}}\left|\left(1+|x|^{2}\right)^{k}\right|\left|D^{\alpha} f(x)\right|
$$

is finite, for all $k \in \mathbb{N}$. The Schwarz space, denoted as $\mathcal{S}$, is the set of all rapidly decreasing functions, together with the family of seminorms $\left(p_{k}\right)_{k \geq 0}$.

In this setting, $\mathcal{S}$ is a Fréchet space [1, Theorem 7.4]; that is, it is a complete space with respect to the topology given by the family of seminorms: for any sequence $\left(f_{m}\right)_{m \geq 0} \subset \mathcal{S}$ such that

$$
p_{k}\left(f_{m}-f_{m^{\prime}}\right) \stackrel{m, m^{\prime} \rightarrow \infty}{\longrightarrow} 0, \forall k \in \mathbb{N},
$$

there exists $f \in \mathcal{S}$ such that

$$
p_{k}\left(f_{m}-f\right) \stackrel{m \rightarrow \infty}{\longrightarrow} 0, \forall k \in \mathbb{N} .
$$

Of course, any function $f \in \mathcal{S}$ is also in any $L^{p}$ for $1 \leq p \leq \infty$, and this inclusion is continuous. In particular, functions in $\mathcal{S}$ admit operations such as the convolution and the Fourier transform. Actually, the space $\mathcal{S}$ is dense in $L^{p}$ for $p<\infty$, and if $p=\infty$, the closure of $\mathcal{S}$ is $L_{0}^{\infty} \cap C$, where $C$ denotes the set of all continuous functions. 
Definition 1.19. The dual space of $\mathcal{S}$, which is the space of all linear and continuous functionals from $\mathcal{S}$ to $\mathbb{R}$, is denoted as $\mathcal{S}^{\prime}$, and it is called the space of tempered distributions.

Since $\mathcal{S}$ is a Fréchet space, we can characterize continuity in terms of the family of seminorms: a linear functional $u: \mathcal{S} \rightarrow \mathbb{R}$ is continuous if and only if there is $N \in \mathbb{N}$ such that

$$
|u(f)| \lesssim p_{N}(f)
$$

for all $f \in \mathcal{S}[1$, Theorem 3.4].

There is a way to extend to $\mathcal{S}^{\prime}$ certain operations or transformations that belong to the theory of calculus with functions:

i) Multiplications: given $f \in \mathcal{S}$ and $u \in \mathcal{S}^{\prime}$, the product of $f$ and $u$ is a distribution $f u \in \mathcal{S}^{\prime}$ given by

$$
f u(g)=u(f g), \forall g \in \mathcal{S} .
$$

ii) Derivatives: given $\alpha \in \mathbb{N}^{n}$, and $u \in \mathcal{S}^{\prime}$, the derivative of $u$ of order $\alpha$ is another distribution $D^{\alpha} u \in \mathcal{S}^{\prime}$ such that

$$
D^{\alpha} u(f)=(-1)^{|\alpha|} u\left(D^{\alpha} f\right), \forall f \in \mathcal{S} .
$$

This is well defined because if $f \in \mathcal{S}$, then $D^{\alpha} f \in \mathcal{S}$, for any $\alpha \in \mathbb{N}^{n}$.

iii) Convolution: given $f \in \mathcal{S}$ and $u \in \mathcal{S}^{\prime}$, the convolution of $u$ with $f$ is a function $u * f \in \mathcal{S}$ defined as

$$
(u * f)(x)=u\left(\tau_{x} \tilde{f}\right), \forall x \in \mathbb{R}^{n}
$$

where $\tau_{x} g(y)=g(y-x)$ and $\tilde{f}(x)=f(-x)$, for any functions $f, g \in \mathcal{S}$.

iv) Fourier transform: given $u \in \mathcal{S}^{\prime}$, the Fourier transform of $u$ is another distribution $\widehat{u} \in \mathcal{S}^{\prime}$ such that

$$
\widehat{u}(f)=u(\widehat{f}), \forall f \in \mathcal{S} .
$$

The Fourier transform of a distribution is well defined because if $f \in \mathcal{S}$, then $\widehat{f} \in \mathcal{S}$ as well. Actually, in $\mathcal{S}$ (and thus in $\mathcal{S}^{\prime}$ ) the Fourier transform is a bijective continuous map $\mathcal{F}: \mathcal{S} \rightarrow \mathcal{S}$ such that $\mathcal{F}^{4}=i d$ [1, Theorem 7.5]. Since $\mathcal{F}$ is bijective and $\mathcal{F}^{4}=i d$, it means that its inverse $\mathcal{F}^{-1}$ is just $\mathcal{F}^{-1}=\mathcal{F}^{3}$. In particular, $\mathcal{F}^{-1}$ is also continuous. This inverse map is known as the inverse Fourier transform, which will be denoted as $\breve{g}$, for any $g \in \mathcal{S}$, and it is given by

$$
\check{g}(x)=\int_{\mathbb{R}^{n}} g(\xi) e^{2 \pi i \xi \cdot x} d \xi, \forall x \in \mathbb{R}^{n} .
$$

We note that the fact that $\mathcal{F}$ is a bijection on $\mathcal{S}$, together with together with Plancherel's theorem and the fact that $\mathcal{S}$ is dense in $L^{2}$, allows us to conclude that $\mathcal{F}$ is also a bijection on $L^{2}$. 
We point out some important properties regarding all of the previous operations [1, Chapter 6]. If $f, g \in \mathcal{S}, \alpha \in \mathbb{N}^{n}$, and $u$ is either a function in $\mathcal{S}$ or a distribution in $\mathcal{S}^{\prime}$, then:

i) Leibniz rule: $D^{\alpha}(f u)=\sum_{\beta \leq \alpha} c_{\alpha, \beta} D^{\beta} f D^{\alpha-\beta} u$.

ii) $D^{\alpha}(u * f)=D^{\alpha} u * f=u * D^{\alpha} f$.

iii) $(u * f) * g=u *(f * g)=(u * g) * f$.

iv) $\widehat{u * f}=\widehat{f} \widehat{u}$, and $\widehat{f} * \widehat{u}=\widehat{f u}$.

\subsection{Sobolev spaces}

Sobolev spaces are a way of extending the notion of derivative to functions that might not be differentiable in a classical sense. Notice that, for any $1 \leq p \leq \infty$, a function $f \in L^{p}$ might be regarded as an element of $\mathcal{S}^{\prime}$, by defining

$$
f(g)=\int_{\mathbb{R}^{n}} f(x) g(x) d x, \forall g \in \mathcal{S} .
$$

Actually, this map from $L^{p}$ to $\mathcal{S}^{\prime}$ is one to one [1, Example 7.8], so we sometimes write $L^{p} \subset \mathcal{S}^{\prime}$. With this, if $\alpha \in \mathbb{N}^{n}$, we might now consider the so called weak derivative of $f$ of order $\alpha$, by differentiating $f$ in the sense of distributions:

$$
D^{\alpha} f(g)=(-1)^{|\alpha|} f\left(D^{\alpha} g\right), \forall g \in \mathcal{S} .
$$

Notice that $D^{\alpha} f$ need not be a distribution given by the integral against a function anymore; that is, there might not be any function $f_{\alpha}$ such that

$$
D^{\alpha} f(g)=\int_{\mathbb{R}^{n}} f_{\alpha}(x) g(x) d x .
$$

If $f_{\alpha}$ exists, though, and $f_{\alpha} \in L^{p}$, then we write $D^{\alpha} f \in L^{p}$, and we consider $D^{\alpha} f=f_{\alpha}$.

Definition 1.20. For $1 \leq p \leq \infty$ and $k \in \mathbb{N}$, the Sobolev space $W^{k, p}$ is the space

$$
W^{k, p}=\left\{f \in L^{p}: D^{\alpha} f \in L^{p} \forall|\alpha| \leq k\right\}
$$

equipped with the norm

$$
\|f\|_{k, p}=\sum_{|\alpha| \leq k}\left\|D^{\alpha} f\right\|_{p}
$$

$W^{k, p}$ is a Banach space for any $1 \leq p \leq \infty$ and $k \in \mathbb{N}$, and it is actually a Hilbert space when $p=2$. It is also clear that $W^{k, p}$ is continuously included in $L^{p}$. 


\section{Chapter 2}

\section{Multipliers}

We start now to review the main concepts regarding the theory of translation invariant operators. Our goal is to study tools that can help us to determine boundedness of operators of this kind between $L^{p}$ spaces. For this purpose, we base our initial discussion on the classical article due to Hörmander [8], where some basic theorems regarding these questions are given. We will see an important characterization of these objects, which is essentially that any of these operators can be expressed as a convolution against a tempered distribution.

Thanks to this characterization, one can translate the problem of boundedness of translation invariant operators into a problem of studying certain classes of tempered distributions, or even better, the associated classes of Fourier transforms of these distributions, called multipliers. These classes satisfy nice symmetry properties that are useful when proving the boundedness of translation invariant operators in several $L^{p}$ spaces. But, most importantly, it turns out that every multiplier associated with a bounded operator of this kind from $L^{p}$ into itself must actually be a bounded function. This further reduces the problem of studying boundedness to a problem on giving conditions on functions of $L^{\infty}$.

\subsection{First definitions}

We start with a definition that has already appeared several times in the previous chapter.

Definition 2.1. For any $f \in L^{p}$, and $h \in \mathbb{R}^{n}$, the translation of $f$ by $h$ is the function $\tau_{h} f \in L^{p}$ given by

$$
\tau_{h} f(x)=f(x-h) .
$$

It is clear that if $h \in \mathbb{R}^{n}$, then $\left\|\tau_{h} f\right\|_{p}=\|f\|_{p}$, for all $f \in L^{p}$. In particular, $\tau_{h}$ is a bounded and linear operator from $L^{p}$ to $L^{p}$ with $\left\|\tau_{h}\right\|=1$.

Definition 2.2. Let $A$ a linear operator. We say that $A$ is translation invariant if, for all $h \in \mathbb{R}^{n}$, we have

$$
\tau_{h} A=A \tau_{h}
$$


Examples 2.3. We see some examples of translation invariant operators:

i) Let $f \in L^{1}$, and $1 \leq p \leq \infty$. Let $A_{f}$ be the operator given by $A_{f} g=f * g$, for any $g \in L^{p}$. This is a linear operator which is strong type $(p, p)$ (see Section 1.3), and it is translation invariant. Indeed, for $h \in \mathbb{R}^{n}$,

$$
\begin{aligned}
A_{f} \tau_{h} g(x) & =\left(f * \tau_{h} g\right)(x)=\int_{\mathbb{R}^{n}} f(y) \tau_{h} g(x-y) d y \\
& =\int_{\mathbb{R}^{n}} f(y) g(x-h-y) d y=(f * g)(x-h) \\
& =A_{f} g(x-h)=\tau_{h} A_{f} g(x) .
\end{aligned}
$$

ii) For $n=1$, the Hilbert transform $H$ is the linear operator defined as

$$
H g(x)=\operatorname{p} \cdot \mathrm{v} \cdot \frac{1}{\pi} \int_{\mathbb{R}} \frac{g(y)}{x-y} d y,
$$

where p.v. denotes the principal value of the integral. Using a similar technique as before, one sees that $H$ is a translation invariant operator. Nevertheless, notice that $H$ is not given by any of the former operators, although it can also be thought as the convolution again the distribution

$$
h(g)=\operatorname{p.v} \cdot \frac{1}{\pi} \int_{\mathbb{R}} \frac{g(y)}{y} d y .
$$

The Hilbert transform is strong type $(p, p)$ when $1<p<\infty$. We will prove this fact later (see Example 2.13).

In spite of what one could think at first, translation invariant operators actually satisfy quite strong properties. First, regarding their structure, they form a Banach space.

Lemma 2.4. Given $1 \leq p, q \leq \infty$, the set of linear and bounded translation invariant operators from $L^{p}$ to $L^{q}$ is a Banach space.

Proof. $\mathcal{L}\left(L^{p}, L^{q}\right)$ is a Banach space, so we only need to see that translation invariant operators are a closed subset of it. That is, if $\left(A_{k}\right)_{k \geq 0}$ is a sequence of translation invariant operators convergent to a bounded and linear operator $A$, then $A$ is also translation invariant.

Take $f \in L^{p}$. Since $A_{k} \stackrel{n \rightarrow \infty}{\longrightarrow} A$, given $\varepsilon>0$ there is $k_{0} \in \mathbb{N}$ large enough so that $\left\|A_{k}-A\right\|<\varepsilon /\left(2\|f\|_{p}\right)$ if $k \geq k_{0}$. Therefore,

$$
\begin{aligned}
\left\|\tau_{h} A f-A \tau_{h} f\right\|_{q} & =\left\|\tau_{h} A f-\tau_{h} A_{k} f+\tau_{h} A_{k} f-A \tau_{h} f\right\|_{q} \\
& =\left\|\tau_{h} A f-\tau_{h} A_{k} f+A_{k} \tau_{h} f-A \tau_{h} f\right\|_{q} \\
& \leq\left\|\tau_{h} A f-\tau_{h} A_{k} f\right\|_{q}+\left\|A_{k} \tau_{h} f-A \tau_{h} f\right\|_{q} \\
& =\left\|\tau_{h}\left(A f-A_{k}\right) f\right\|_{q}+\left\|\left(A_{k}-A\right) \tau_{h} f\right\|_{q} \\
& \leq\left\|\left(A f-A_{k}\right) f\right\|_{q}+\left\|A_{k}-A\right\|\left\|\tau_{h} f\right\|_{p} \\
& \leq\left\|A-A_{k}\right\|\|f\|_{p}+\left\|A-A_{k}\right\|\|f\|_{p} \\
& =2\left\|A-A_{k}\right\|\|f\|_{p}<\varepsilon
\end{aligned}
$$


if $k \geq k_{0}$. But this is true for any $\varepsilon>0$, so $\tau_{h} A f=A \tau_{h} f$, and because $f$ is arbitrary, $A$ is translation invariant.

Another surprising result that we get for translation invariant operators is that it only makes sense to study them for certain values of $p$ and $q$.

Lemma 2.5. Let $p>q$, and let $A: L^{p} \rightarrow L^{q}$ be a linear translation invariant operator. If $p<\infty$, then $A \equiv 0$. On the other hand, if $p=\infty$, then $\left.A\right|_{L_{0}^{\infty}} \equiv 0$.

Proof. Take $p<\infty$. Since $A$ is bounded, there exists some constant $C>0$ such that

$$
\|A f\|_{q} \leq C\|f\|_{p}
$$

for all $f \in L^{p}$. We shall see that we can improve this bound as much as we want, so that the conclusion follows. To do so, we claim first that

$$
\left\|f+\tau_{h} f\right\|_{p} \stackrel{|h| \rightarrow \infty}{\longrightarrow} 2^{1 / p}\|f\|_{p}
$$

Indeed, given $\varepsilon>0$ write $f$ as $f=f_{1}+f_{2}$ so that $f_{1}$ has compact support and $\left\|f_{2}\right\|_{p}<\varepsilon /\left(2+2^{1 / p}\right)$. For $|h|$ large enough, $f_{1}$ and $\tau_{h} f_{1}$ will have disjoint supports, so that

$$
\begin{aligned}
\left\|f_{1}+\tau_{h} f_{1}\right\|_{p} & =\left(\int_{\mathbb{R}^{n}}\left|f_{1}(x)+\tau_{h} f_{1}(x)\right|^{p} d x\right)^{1 / p} \\
& =\left(\int_{\mathbb{R}^{n}}\left|f_{1}(x)\right|^{p} d x+\int_{\mathbb{R}^{n}}\left|\tau_{h} f_{1}(x)\right|^{p} d x\right)^{1 / p} \\
& =2^{1 / p}\left\|f_{1}\right\|_{p} .
\end{aligned}
$$

With this,

$$
\begin{aligned}
\left|\left\|f+\tau_{h} f\right\|_{p}-2^{1 / p}\|f\|_{p}\right| & =\left|\left\|f+\tau_{h} f\right\|_{p}-\left\|f_{1}+\tau_{h} f_{1}\right\|_{p}+2^{1 / p}\left\|f_{1}\right\|_{p}-2^{1 / p}\|f\|_{p}\right| \\
& \leq\left|\left\|f+\tau_{h} f\right\|_{p}-\left\|f_{1}+\tau_{h} f_{1}\right\|_{p}\right|+2^{1 / p}\left|\left\|f_{1}\right\|_{p}-\|f\|_{p}\right| \\
& \leq\left\|f_{2}+\tau_{h} f_{2}\right\|_{p}+2^{1 / p}\left\|f_{2}\right\|_{p} \leq \frac{2 \varepsilon}{2+2^{1 / p}}+\frac{2^{1 / p} \varepsilon}{2+2^{1 / p}}=\varepsilon
\end{aligned}
$$

so the claim holds. This actually implies as well, since $A$ is translation invariant, that

$$
\left\|A\left(f+\tau_{h} f\right)\right\|_{q}=\left\|A f+A \tau_{h} f\right\|_{q}=\left\|A f+\tau_{h} A f\right\|_{q} \stackrel{|h| \rightarrow \infty}{\longrightarrow} 2^{1 / q}\|A f\|_{q} .
$$

All in all, because

$$
\left\|A\left(f+\tau_{h} f\right)\right\|_{q} \leq C\left\|f+\tau_{h} f\right\|_{p},
$$

taking the limit when $|h| \rightarrow \infty$ yields

$$
2^{1 / q}\|A f\|_{q} \leq C 2^{1 / p}\|f\|_{p},
$$


SO

$$
\|A f\|_{q} \leq \frac{C}{2^{1 / q-1 / p}}\|f\|_{p}
$$

which is an improved version of $(2.1)$ because $1 / q>1 / p$. But this can now be iterated to make the constant in the estimate arbitrarily small, so we conclude that $A \equiv 0$. The same argument works for $p=\infty$, and the hypothesis $f \in L_{0}^{\infty}$ is necessary so that the decomposition $f=f_{1}+f_{2}$ can be done.

In virtue of this result, we shall assume from now on that $p \leq q$.

\subsection{The spaces $L_{p}^{q}$ and $M_{p}^{q}$}

As we have mentioned, being translation invariant is in fact a very strong condition, and because of this, translation invariant operators admit a very specific characterization. Notice that, for instance, all the operators in Examples 2.3 are related somehow with convolutions. We are going to prove that this is the general case for this class of operators.

Theorem 2.6. Let $1 \leq p \leq q \leq \infty$, and let $A: L^{p} \rightarrow L^{q}$ be a linear translation invariant operator. Then, there exists a distribution $u \in \mathcal{S}^{\prime}$ such that $A f=u * f$, for all $f \in \mathcal{S}$.

Proof. Take $p<\infty$. Given $f \in \mathcal{S}$ and $u \in \mathcal{S}^{\prime}$, recall that $(u * f)(x)=u\left(\tau_{x} \tilde{f}\right)$, where $\tilde{f}(x)=f(-x)$, for all $x \in \mathbb{R}^{n}$. If we want the result to hold, then notice that we should have $A f(0)=u(\tilde{f})$. That is, we must define $u$ as

$$
u(f)=A \tilde{f}(0)
$$

and so, since $u$ is clearly linear, we must see now that it is a continuous functional on $\mathcal{S}$; that is, there is $m \in \mathbb{N}$ so that $|u(f)| \lesssim p_{m}(f)$, for all $f \in \mathcal{S}$. First, let us see that

$$
D^{\alpha}(A f)=A\left(D^{\alpha} f\right) .
$$

To prove this, it suffices to do it for one derivative, and then proceed iteratively. Thus, set $f_{h}(x)=f\left(x_{1}+h, \bar{x}\right)$ where $\bar{x}=\left(x_{2}, \ldots, x_{n}\right)$, and let $v=A f$. Since $A$ is translation invariant, we have that $v_{h}=A f_{h}$, so

$$
A\left(\frac{f_{h}-f}{h}\right)=\frac{v_{h}-v}{h} .
$$

Therefore, and since $A$ is bounded, we have

$$
\left\|A\left(\partial_{x_{1}} f\right)-\frac{v_{h}-v}{h}\right\|_{q}=\left\|A\left(\partial_{x_{1}} f-\frac{f_{h}-f}{h}\right)\right\|_{q} \leq C\left\|\partial_{x_{1}} f-\frac{f_{h}-f}{h}\right\|_{p},
$$


and we shall now prove that $\left\|\partial_{x_{1}} f-\left(f_{h}-f\right) / h\right\|_{p} \rightarrow 0$ when $h \rightarrow 0$. This is so because

$$
\begin{aligned}
\left|\frac{\partial f}{\partial x_{1}}(x)-\frac{f_{h}(x)-f(x)}{h}\right| & =\left|\frac{\partial f}{\partial x_{1}}(x)-\frac{1}{h} \int_{0}^{h} \frac{\partial f}{\partial x_{1}}\left(x_{1}+\xi, \bar{x}\right) d \xi\right| \\
& =\left|\frac{1}{h} \int_{0}^{h} \frac{\partial f}{\partial x_{1}}(x) d \xi-\frac{1}{h} \int_{0}^{h} \frac{\partial f}{\partial x_{1}}\left(x_{1}+\xi, \bar{x}\right) d \xi\right| \\
& \leq \frac{1}{h} \int_{0}^{h}\left|\frac{\partial f}{\partial x_{1}}(x)-\frac{\partial f}{\partial x_{1}}\left(x_{1}+\xi, \bar{x}\right)\right| d \xi \\
& \leq \frac{1}{h}\left(\int_{0}^{h} d \xi\right)^{1 / p^{\prime}}\left(\int_{0}^{h}\left|\frac{\partial f}{\partial x_{1}}(x)-\frac{\partial f}{\partial x_{1}}\left(x_{1}+\xi, \bar{x}\right)\right|^{p} d \xi\right)^{1 / p} \\
& =\left(\frac{1}{h} \int_{0}^{h}\left|\frac{\partial f}{\partial x_{1}}(x)-\frac{\partial f}{\partial x_{1}}\left(x_{1}+\xi, \bar{x}\right)\right|^{p} d \xi\right)^{1 / p},
\end{aligned}
$$

meaning that

$$
\begin{aligned}
\left\|\partial_{x_{1}} f-\frac{f_{h}-f}{h}\right\|_{p}^{p} & =\int_{\mathbb{R}^{n}}\left|\frac{\partial f}{\partial x_{1}}(x)-\frac{f_{h}(x)-f(x)}{h}\right|^{p} d x \\
& \leq \frac{1}{h} \int_{\mathbb{R}^{n}} \int_{0}^{h}\left|\frac{\partial f}{\partial x_{1}}(x)-\frac{\partial f}{\partial x_{1}}\left(x_{1}+\xi, \bar{x}\right)\right|^{p} d \xi d x \\
& =\frac{1}{h} \int_{0}^{h} \int_{\mathbb{R}^{n}}\left|\frac{\partial f}{\partial x_{1}}(x)-\frac{\partial f}{\partial x_{1}}\left(x_{1}+\xi, \bar{x}\right)\right|^{p} d x d \xi \\
& =\frac{1}{h} \int_{0}^{h}\left\|\partial_{x_{1}} f-\left(\partial_{x_{1}} f\right)_{\xi}\right\|_{p}^{p} d \xi,
\end{aligned}
$$

but given $\varepsilon>0$ there exists $h>0$ small enough so that

$$
\left\|\partial_{x_{1}} f-\left(\partial_{x_{1}} f\right)_{\xi}\right\|_{p}<\varepsilon
$$

if $0<\xi<h$ because $\partial_{x_{1}} f \in L^{p}$ and $1 \leq p<\infty$ [4, Proposition 8.5], so finally

$$
\left\|\partial_{x_{1}} f-\frac{f_{h}-f}{h}\right\|_{p}<\left(\frac{1}{h} \int_{0}^{h} \varepsilon^{p} d \xi\right)^{1 / p}=\varepsilon
$$

Expression (2.2) really tells us that if $f \in \mathcal{S}$, then $A f \in W^{n, q}$. But $W^{n, q} \hookrightarrow C$ (see [8, Lemma 1.1]), so in particular

$$
\begin{aligned}
|u(f)| & =|A \tilde{f}(0)| \lesssim \sum_{|\alpha| \leq n}\left\|D^{\alpha}(A \tilde{f})\right\|_{q}=\sum_{|\alpha| \leq n}\left\|A\left(D^{\alpha} \tilde{f}\right)\right\|_{q} \lesssim \sum_{|\alpha| \leq n}\left\|D^{\alpha} \tilde{f}\right\|_{p} \\
& =\sum_{|\alpha| \leq n}\left(\int_{\mathbb{R}^{n}} \frac{1}{\left(1+|x|^{2}\right)^{n}}\left|\left(1+|x|^{2}\right)^{n} D^{\alpha} \tilde{f}(x)\right|^{p} d x\right)^{1 / p} \lesssim p_{n}(\tilde{f})=p_{n}(f),
\end{aligned}
$$

and hence $u$ is indeed a continuous functional on $\mathcal{S}$, and the proof is complete. The same argument works for $p=\infty$, writing down the proper norm in that case. It is important to notice that (2.3) would not be true in general for the supremum norm, but it does hold in this case because $\partial_{x_{1}} f$ is uniformly continuous. 
Let $u \in \mathcal{S}^{\prime}$, such that $u * g \in L^{q}$, for all $g \in \mathcal{S}$. If $p<\infty$, since $\mathcal{S}$ is dense in $L^{p}$, the distribution $u$ defines uniquely a translation invariant operator $A$ as

$$
\text { Af }=L^{q}-\lim _{k \rightarrow \infty} u * f_{k},
$$

for any $f \in L^{p}$, where $\left(f_{k}\right)_{k \geq 0} \subset \mathcal{S}$ so that $f_{k} \stackrel{L^{p}}{\rightarrow} f$. Thus, this previous theorem tells us that studying translation invariant operators is essentially studying certain sets of tempered distributions. In particular, if we want to prove that a linear translation invariant operator is bounded from $L^{p}$ to $L^{q}$, it suffices to prove its boundedness for functions in $\mathcal{S}$ and then extend the operator by density, so it is enough to see that

$$
\|u * f\|_{q} \lesssim\|f\|_{p}, \forall f \in \mathcal{S}
$$

where $u$ is the associated tempered distribution. This fact motivates the following definition.

Definition 2.7. Given $1 \leq p \leq q \leq \infty$, we will denote as $L_{p}^{q}$ the space

$$
L_{p}^{q}=\left\{u \in \mathcal{S}^{\prime}:\|u * f\|_{q} \lesssim\|f\|_{p} \forall f \in \mathcal{S}\right\} .
$$

Moreover, for a distribution $u \in L_{p}^{q}$, we will write

$$
L_{p}^{q}(u)=\sup _{f \in \mathcal{S}} \frac{\|u * f\|_{q}}{\|f\|_{p}} .
$$

By Theorem 2.6, the space $L_{p}^{q}$ is isomorphic to the space of translation invariant operators, which is Banach space by Lemma 2.4. Thus, $L_{p}^{q}$ is a Banach space.

Since $\widehat{u * f}=\widehat{u} \widehat{f}$ for $u \in \mathcal{S}^{\prime}$ and $f \in \mathcal{S}$, it makes sense to consider the Fourier transform of the elements of the sets $L_{p}^{q}$. These new objects are called multipliers.

Definition 2.8. Given $1 \leq p \leq q \leq \infty$, the set of multipliers of type $(p, q)$ is the set

$$
M_{p}^{q}=\left\{\widehat{u}: u \in L_{p}^{q}\right\}
$$

and we write $M_{p}^{q}(\widehat{u})=L_{p}^{q}(u)$.

We will see soon that studying the spaces of multipliers is more convenient than doing it with the spaces $L_{p}^{q}$, at least when $p=q$.

\subsection{Properties of $L_{p}^{q}$ and $M_{p}^{q}$}

We now want to improve our understanding on $L_{p}^{q}$ and $M_{p}^{q}$, so we are going to review well known properties of those spaces and even some characterizations that occur in some particular cases. Our first result can be thought as a symmetry property that these spaces satisfy. 
Theorem 2.9. Let $u \in S^{\prime}$ be a non-trivial tempered distribution. Then:

i) The set

$$
\mathcal{L}_{u}=\left\{(s, t) \in \mathbb{R}^{2}: u \in L_{1 / s}^{1 / t}\right\}
$$

is a convex subset of the triangle $0 \leq t \leq s \leq 1$ which is symmetric with respect to the line $s+t=1$.

ii) The function $L_{1 / s}^{1 / t}(u)$ is also symmetric in $\mathcal{L}_{u}$ with respect to $s+t=1$.

iii) For any $(s, t),(r, w) \in \mathcal{L}_{u}$, and $\theta \in(0,1)$, it holds

$$
L_{p}^{q}(u) \leq\left[L_{1 / s}^{1 / t}(u)\right]^{1-\theta}\left[L_{1 / r}^{1 / w}(u)\right]^{\theta}
$$

where

$$
\begin{aligned}
& \frac{1}{p}=(1-\theta) s+\theta r \\
& \frac{1}{q}=(1-\theta) t+\theta w .
\end{aligned}
$$

Proof. It is easy to see that $\mathcal{L}_{u}$ is a subset of the given triangle, for $1 \leq 1 / s \leq 1 / t \leq \infty$, because $u$ is non-trivial. To prove symmetry, take $(s, t) \in \mathcal{L}_{u}$, and consider its symmetric point $\left(t^{\prime}, s^{\prime}\right)=(1-t, 1-s)$ with respect to $s+t=1$. It turns out that if $p=1 / s$ and $q=1 / t$, then $q^{\prime}=1 / t^{\prime}$ and $p^{\prime}=1 / s^{\prime}$. Since

$$
\|u * f\|_{1 / t} \leq C\|f\|_{1 / s}
$$

applying Hölder's inequality leads to

$$
|((u * f) * g)(0)| \leq\|(u * f) g\|_{1} \leq\|u * f\|_{1 / t}\|g\|_{1 / t^{\prime}} \leq C\|f\|_{1 / s}\|g\|_{1 / t^{\prime}} .
$$

In particular, if $\|f\|_{1 / s}=1$, then

$$
|((u * f) * g)(0)| \leq C\|g\|_{1 / t^{\prime}}
$$

But notice that $|((u * f) * g)(0)|=|((u * g) * f)(0)|$ because convolution is associative and commutative, and

$$
\|u * g\|_{1 / s^{\prime}}=\sup _{\|f\|_{1 / s}=1}\left|\int_{\mathbb{R}^{n}}(u * g)(x) f(x) d x\right|=\sup _{\|f\|_{1 / s}=1}|((u * g) * \tilde{f})(0)|,
$$

so we conclude

$$
\|u * g\|_{1 / s^{\prime}} \leq C\|g\|_{1 / t^{\prime}}
$$

and hence $\left(t^{\prime}, s^{\prime}\right) \in \mathcal{L}_{u}$. Moreover, $L_{1 / s}^{1 / t}(u)=L_{1 / t^{\prime}}^{1 / s^{\prime}}(u)$ because $(s, t)$ and $\left(t^{\prime}, s^{\prime}\right)$ can be interchanged in the previous argument. Finally, convexity and (2.4) follow from Riesz-Thorin convexity theorem [5, Theorem 1.3.4]. 
Let us see some easy consequences of Theorem 2.9 on the $L_{p}^{q}$ spaces for certain choices of $p$ and $q$, that actually characterize these spaces.

Corollary 2.10. If $1 \leq p \leq q \leq \infty$, then $L_{p}^{q}=L_{q^{\prime}}^{p^{\prime}}$.

Proof. Set $p=1 / s, q=1 / t$. Then $q^{\prime}=1 /(1-t)$ and $p^{\prime}=1 /(1-s)$. But the point $(1-t, 1-s)$ is the symmetric point to $(s, t)$ with respect to the line $s+t=1$. Thus, by the previous theorem, $u \in L_{p}^{q}$ if and only if $u \in L_{q^{\prime}}^{p^{\prime}}$.

Corollary 2.11. For $1 \leq p<\infty^{1}$, and with the corresponding equalities of norms, we have

$$
L_{p}^{\infty}=L_{1}^{p^{\prime}}=L^{p^{\prime}}
$$

Proof. If $p<\infty$, by Corollary 2.10 is clear that $L_{p}^{\infty}=L_{1}^{p^{\prime}}$, so it is left to see that $L_{p}^{\infty}=L^{p^{\prime}}$. The inclusion $L_{p}^{\infty} \subset L^{p^{\prime}}$ is true because if $u \in L_{p}^{\infty}$, then

$$
|u(f)|=|(u * f)(0)| \leq\|u * f\|_{\infty} \leq\|u\|\|f\|_{p},
$$

for all $f \in \mathcal{S}$, and so by density of $\mathcal{S}$ in $L^{p}, u$ is an element of $\left(L^{p}\right)^{\prime}$. On the other hand, for $u \in\left(L^{p}\right)^{\prime}$, we have

$$
\|u * f\|_{\infty}=\sup _{x \in \mathbb{R}^{n}}\left|u\left(\tau_{x} \tilde{f}\right)\right| \leq\|u\| \sup _{x \in \mathbb{R}^{n}}\left\|\tau_{x} \tilde{f}\right\|_{p}=\|u\|\|f\|_{p}, \forall f \in \mathcal{S},
$$

so $u \in L_{p}^{\infty}$, and we conclude $L_{p}^{\infty}=\left(L^{p}\right)^{\prime}$. Of course, by definition, it is clear that $L_{p}^{\infty}(u)=\|u\|$. But now recall that $\left(L^{p}\right)^{\prime}$ is isometrically isomorphic to $L^{p^{\prime}}$, so the result follows.

The diagonal cases $p=q$ will be the ones of greater interest in this work, and we will devote the rest of this chapter to give some insight of their structure. Theorem 2.9 gives in this case an easy but interesting consequence on how this spaces are actually included one inside the other. In a way, as $p \rightarrow 2$, the space $L_{p}^{p}$ gets bigger.

Corollary 2.12. For any $q \leq p<2$ or $q \geq p>2$, we have $L_{q}^{q} \subset L_{p}^{p} \subset L_{2}^{2}$ and

$$
L_{2}^{2}(u) \leq L_{p}^{p}(u) \leq L_{q}^{q}(u), \forall u \in L_{q}^{q}
$$

Proof. By Corollary 2.10, if $u \in L_{q}^{q}$, then $u \in L_{q^{\prime}}^{q^{\prime}}$, and by the convexity of $\mathcal{L}_{u}$ given by Theorem 2.9, $u \in L_{p}^{p}$. Of course, we also have $u \in L_{2}^{2}$, for $(1 / 2,1 / 2)$ is always the middle point of the segment between $(1 / q, 1 / q)$ and $\left(1 / q^{\prime}, 1 / q^{\prime}\right)$. Moreover, if we write

$$
\frac{1}{2}=\frac{1}{2 p}+\frac{1}{2 p^{\prime}}
$$

and

$$
\frac{1}{p}=\frac{1-\theta}{q}+\frac{\theta}{q^{\prime}}
$$

\footnotetext{
${ }^{1}$ For $p=\infty$, it holds $L_{\infty}^{\infty}=L_{1}^{1}=M$, where $M$ is the space of signed bounded measures on $\mathcal{B}\left(\mathbb{R}^{n}\right)$. We refer to $[5$, Theorem 2.5.8] for a proof of this fact.
} 
for some $\theta \in(0,1)$, then again by Theorem 2.9 we have

$$
\begin{aligned}
L_{2}^{2}(u) & \leq\left[L_{p}^{p}(u)\right]^{1 / 2}\left[L_{p^{\prime}}^{p^{\prime}}(u)\right]^{1 / 2}=\left[L_{p}^{p}(u)\right]^{1 / 2}\left[L_{p}^{p}(u)\right]^{1 / 2}=L_{p}^{p}(u) \\
& \leq\left[L_{q}^{q}(u)\right]^{1-\theta}\left[L_{q^{\prime}}^{q^{\prime}}(u)\right]^{\theta}=\left[L_{q}^{q}(u)\right]^{1-\theta}\left[L_{q}^{q}(u)\right]^{\theta}=L_{q}^{q}(u) .
\end{aligned}
$$

Example 2.13 (The Hilbert transform). The previous results are very useful tools for determining boundedness of translation invariant operators. We can use them, for instance, to see that the Hilbert transform, defined in Examples 2.3, is strong type $(p, p)$ for $1<p<\infty$.

We can start by seeing that $H$ is bounded from $L^{2}$ to $L^{2}$. As we have already said, the Hilbert transform can be expressed as

$$
H(f)=h * f
$$

where

$$
h(f)=\operatorname{p} \cdot \mathrm{v} \cdot \frac{1}{\pi} \int_{\mathbb{R}} \frac{f(y)}{y} d y
$$

for all $f \in \mathcal{S}$. Since we are looking for boundedness in $L^{2}$, it might be useful to consider the Fourier transform of $h$ and use Plancherel's theorem. We have that

$$
\begin{aligned}
\widehat{h}(f) & =h(\widehat{f})=\operatorname{p.v} \cdot \frac{1}{\pi} \int_{\mathbb{R}} \frac{\widehat{f}(\xi)}{\xi} d \xi=\lim _{\varepsilon \rightarrow 0} \frac{1}{\pi} \int_{\varepsilon<|\xi|<\frac{1}{\varepsilon}} \frac{1}{\xi}\left(\int_{\mathbb{R}} f(x) e^{-2 \pi i \xi x} d x\right) d \xi \\
& =\lim _{\varepsilon \rightarrow 0} \frac{1}{\pi} \int_{\mathbb{R}} \int_{\mathbb{R}} \frac{1}{\bar{\xi}} \chi_{\varepsilon<|\xi|<\frac{1}{\varepsilon}}(\xi) f(x) e^{-2 \pi i \xi x} d x d \xi \\
& =\lim _{\varepsilon \rightarrow 0} \frac{1}{\pi} \int_{\mathbb{R}} f(x)\left(\int_{\varepsilon<|\xi|<\frac{1}{\varepsilon}} \frac{1}{\xi} e^{-2 \pi i \xi x} d \xi\right) d x \\
& =\lim _{\varepsilon \rightarrow 0} \frac{-i}{\pi} \int_{\mathbb{R}} f(x)\left(\int_{\varepsilon<|\xi|<\frac{1}{\varepsilon}} \frac{1}{\xi} \sin (2 \pi \xi x) d \xi\right) d x .
\end{aligned}
$$

If $x \in \mathbb{R}$ is fixed, the integral between parenthesis in the last equality is convergent when $\varepsilon \rightarrow 0$, because

$$
\int_{0}^{\infty} \frac{1}{\xi} \sin (2 \pi \xi x) d \xi=\int_{0}^{1} \frac{1}{\xi} \sin (2 \pi \xi x) d \xi+\int_{1}^{\infty} \frac{1}{\xi} \sin (2 \pi \xi x) d \xi
$$

and the first integral is convergent since $\sin (2 \pi \xi x) / \xi \sim 2 \pi x$ when $|\xi| \rightarrow 0$, while the second one is also convergent by Dirichlet's test. Thus, if we define

$$
g_{\varepsilon}(x)=f(x) \int_{\varepsilon<|\xi|<\frac{1}{\varepsilon}} \frac{1}{\xi} \sin (2 \pi \xi x) d \xi, \forall x \in \mathbb{R},
$$

then $\left(g_{\varepsilon}\right)_{\varepsilon>0}$ is pointwise convergent to the function

$$
g(x)=f(x) \int_{\mathbb{R}} \frac{1}{\xi} \sin (2 \pi \xi x) d \xi, \forall x \in \mathbb{R}
$$


as $\varepsilon \rightarrow 0$. Moreover, if

$$
M=\sup _{a, b>0}\left|\int_{a<|\xi|<b} \frac{1}{\xi} \sin (2 \pi \xi x) d \xi\right|<\infty,
$$

then

$$
\begin{aligned}
\left|g_{\varepsilon}(x)\right| & =|f(x)|\left|\int_{\varepsilon<|\xi|<\frac{1}{\varepsilon}} \frac{1}{\xi} \sin (2 \pi \xi x) d \xi\right| \\
& =|f(x)|\left|\int_{2 \pi|x| \varepsilon<|t|<\frac{2 \pi|x|}{\varepsilon}} \frac{1}{t} \sin (t) d t\right| \leq M|f(x)|,
\end{aligned}
$$

so by the dominated convergence theorem, $g_{\varepsilon} \stackrel{\varepsilon \rightarrow 0}{\longrightarrow} g$ in $L^{1}$, and

$$
\lim _{\varepsilon \rightarrow 0} \int_{\mathbb{R}} f(x)\left(\int_{\varepsilon<|\xi|<\frac{1}{\varepsilon}} \frac{1}{\xi} \sin (2 \pi \xi x) d \xi\right) d x=\int_{\mathbb{R}} f(x)\left(\int_{\mathbb{R}} \frac{1}{\xi} \sin (2 \pi \xi x) d \xi\right) d x .
$$

Finally, notice that

$$
\int_{\mathbb{R}} \frac{1}{\xi} \sin (2 \pi \xi x) d \xi=\operatorname{sgn}(2 \pi x) \int_{\mathbb{R}} \frac{1}{t} \sin (t) d t=\operatorname{sgn}(x) \int_{\mathbb{R}} \frac{1}{t} \sin (t) d t,
$$

where $\operatorname{sgn}(x)$ denotes the sign of $x$, and it is well known that

$$
\int_{\mathbb{R}} \frac{1}{t} \sin (t) d t=\pi
$$

Thus, we conclude that

$$
\widehat{h}(f)=\int_{\mathbb{R}} f(x)(-i \operatorname{sgn}(x)) d x,
$$

and with this, we can now prove the boundedness of $H$ in $L^{2}$ : given $f \in \mathcal{S}$,

$$
\|\widehat{H(f)}\|_{2}=\|\widehat{h * f}\|_{2}=\|\widehat{h} \widehat{f}\|_{2}=\|\widehat{f}\|_{2}=\|f\|_{2}
$$

that is, $\|\widehat{H(f)}\|_{2}$ is finite and equal to $\|f\|_{2}$, so by Plancherel's theorem,

$$
\|H(f)\|_{2}=\|f\|_{2}, \forall f \in \mathcal{S},
$$

meaning that $H$ is actually an isometry, and thus it is indeed strong type $(2,2)$.

Next step is seeing that $H$ is strong type $\left(2^{k}, 2^{k}\right)$ for any $k \geq 1$. We prove first that

$$
H(f)^{2}=f^{2}+2 H(f H(f)), \forall f \in \mathcal{S},
$$

and to do so, we shall prove the following equivalent identity, obtained after taking the Fourier transform:

$$
(\widehat{h} \widehat{f}) *(\widehat{h} \widehat{f})=\widehat{f} * \widehat{f}+2 \widehat{h}(\widehat{f} *(\widehat{h} \widehat{f})), \forall f \in \mathcal{S} .
$$


Indeed, denote by $R$ the right hand side of the previous identity. Then, given $\xi \in \mathbb{R}$, we have

$$
R(\xi)=\int_{\mathbb{R}} \widehat{f}(y) \widehat{f}(\xi-y) d y+2 \widehat{h}(\xi) \int_{\mathbb{R}} \widehat{f}(y) \widehat{h}(\xi-y) \widehat{f}(\xi-y) d y,
$$

but by commutativity of the convolution, we can also write

$$
R(\xi)=\int_{\mathbb{R}} \widehat{f}(y) \widehat{f}(\xi-y) d y+2 \widehat{h}(\xi) \int_{\mathbb{R}} \widehat{f}(y) \widehat{h}(y) \widehat{f}(\xi-y) d y,
$$

so we have that

$$
R(\xi)=\frac{R(\xi)+R(\xi)}{2}=\int_{\mathbb{R}} \widehat{f}(y) \widehat{f}(\xi-y)[1+\widehat{h}(\xi)(\widehat{h}(y)+\widehat{h}(\xi-y))] d y .
$$

But now, notice that since $\widehat{h}(x)=-i \operatorname{sgn}(x)$, then

$$
\begin{aligned}
1+\widehat{h}(\xi)(\widehat{h}(y)+\widehat{h}(\xi-y)) & =1-i \operatorname{sgn}(\xi)(-i \operatorname{sgn}(y)+-i \operatorname{sgn}(\xi-y)) \\
& =1-\operatorname{sgn}(y) \operatorname{sgn}(\xi)-\operatorname{sgn}(\xi) \operatorname{sgn}(\xi-y) .
\end{aligned}
$$

When $|\xi|>|y|$, then $\operatorname{sgn}(\xi-y)=\operatorname{sgn}(\xi)$, so

$$
\begin{aligned}
1-\operatorname{sgn}(y) \operatorname{sgn}(\xi)-\operatorname{sgn}(\xi) \operatorname{sgn}(\xi-y) & =1-\operatorname{sgn}(y) \operatorname{sgn}(\xi-y)-\operatorname{sgn}(\xi) \operatorname{sgn}(\xi) \\
& =-\operatorname{sgn}(y) \operatorname{sgn}(\xi-y) \\
& =\widehat{h}(y) \widehat{h}(\xi-y) .
\end{aligned}
$$

On the other hand, if $|\xi|<|y|$, then $\operatorname{sgn}(\xi-y)=-\operatorname{sgn}(y)$, so

$$
\begin{aligned}
1-\operatorname{sgn}(y) \operatorname{sgn}(\xi)-\operatorname{sgn}(\xi) \operatorname{sgn}(\xi-y) & =\operatorname{sgn}(y) \operatorname{sgn}(y)+\operatorname{sgn}(\xi-y) \operatorname{sgn}(\xi) \\
& -\operatorname{sgn}(\xi) \operatorname{sgn}(\xi-y) \\
& =-\operatorname{sgn}(y) \operatorname{sgn}(\xi-y) \\
& =\widehat{h}(y) \widehat{h}(\xi-y) .
\end{aligned}
$$

Finally, when $|\xi|=|y|$, we have $\operatorname{sgn}(\xi-y)=(\operatorname{sgn}(\xi)-\operatorname{sgn}(y)) / 2$, so

$$
\begin{aligned}
1-\operatorname{sgn}(y) \operatorname{sgn}(\xi)-\operatorname{sgn}(\xi) \operatorname{sgn}(\xi-y) & =1-\operatorname{sgn}(y) \operatorname{sgn}(\xi)-\operatorname{sgn}(\xi) \frac{\operatorname{sgn}(\xi)-\operatorname{sgn}(y)}{2} \\
& =\frac{1}{2}-\frac{\operatorname{sgn}(y) \operatorname{sgn}(\xi)}{2} \\
& =-\operatorname{sgn}(y) \frac{\operatorname{sgn}(\xi)-\operatorname{sgn}(y)}{2} \\
& =-\operatorname{sgn}(y) \operatorname{sgn}(\xi-y) \\
& =\widehat{h}(y) \widehat{h}(\xi-y) .
\end{aligned}
$$

All in all, we conclude that $1+\widehat{h}(\xi)(\widehat{h}(y)+\widehat{h}(\xi-y))=\widehat{h}(y) \widehat{h}(\xi-y)$, for all $\xi, y \in \mathbb{R}$, and so

$$
R=\int_{\mathbb{R}} \widehat{f}(y) \widehat{f}(\xi-y) \widehat{h}(y) \widehat{h}(\xi-y) d y=((\widehat{h} \widehat{f}) *(\widehat{h} \widehat{f}))(\xi) .
$$


Having (2.5) proved, we can now see that $H$ is strong type $\left(2^{k}, 2^{k}\right)$ for $k \geq 1$. We know the result is true for $k=1$, so let us assume that it is true up to some $k \geq 1$, and let us prove it for $k+1$. If $f \in \mathcal{S}$, and $c_{2^{k}}=\|H\|$ when $H: L^{2^{k}} \rightarrow L^{2^{k}}$, then

$$
\begin{aligned}
\|H(f)\|_{2^{k+1}} & =\left\|H(f)^{2}\right\|_{2^{k}}^{1 / 2} \leq\left(\left\|f^{2}\right\|_{2^{k}}+\| 2 H\left(f(H(f)) \|_{2^{k}}\right)^{1 / 2}\right. \\
& \leq\left(\left\|f^{2}\right\|_{2^{k}}+2 c_{2^{k}}\|f(H(f))\|_{2^{k}}\right)^{1 / 2}
\end{aligned}
$$

and $\|f(H(f))\|_{2^{k}} \leq\|f\|_{2^{k+1}}\|H(f)\|_{2^{k+1}}$ by Hölder's inequality, so after some rearrangement we get

$$
\left(\frac{\|H(f)\|_{2^{k+1}}}{\|f\|_{2^{k+1}}}\right)^{2}-2 c_{2^{k}} \frac{\|H(f)\|_{2^{k+1}}}{\|f\|_{2^{k+1}}}-1 \leq 0 .
$$

The polynomial $p(x)=x^{2}-2 c_{2^{k}} x-1$ is a convex polynomial of degree two with roots $x=c_{2^{k}} \pm \sqrt{c_{2^{k}}^{2}+1}$, so (2.7) really tells us that

$$
\frac{\|H(f)\|_{2^{k+1}}}{\|f\|_{2^{k+1}}} \leq c_{2^{k}}+\sqrt{c_{2^{k}}^{2}+1}
$$

and so $H$ is strong type $\left(2^{k+1}, 2^{k+1}\right)$.

We can now finish to see that $H$ is strong type $(p, p)$, for all $1<p<\infty$. First, since $H$ is strong type $\left(2^{k}, 2^{k}\right)$ for any $k \geq 1$, it means that the points $\left(1 / 2^{k}, 1 / 2^{k}\right)$ belong to the set $\mathcal{L}_{H}$ of Theorem 2.9. All this points lie on the line $x=y$ as shown in figure 2.1. But because $\mathcal{L}_{H}$ is convex, we obtain that any point of the form $(1 / p, 1 / p)$ with $p \geq 2$ is in $\mathcal{L}_{H}$. That is, $H$ is strong type $(p, p)$ for any $2 \leq p<\infty$. Finally, using Corollary 2.10, $H$ is also strong type $\left(p^{\prime}, p^{\prime}\right)$ for any $1<p^{\prime} \leq 2$.

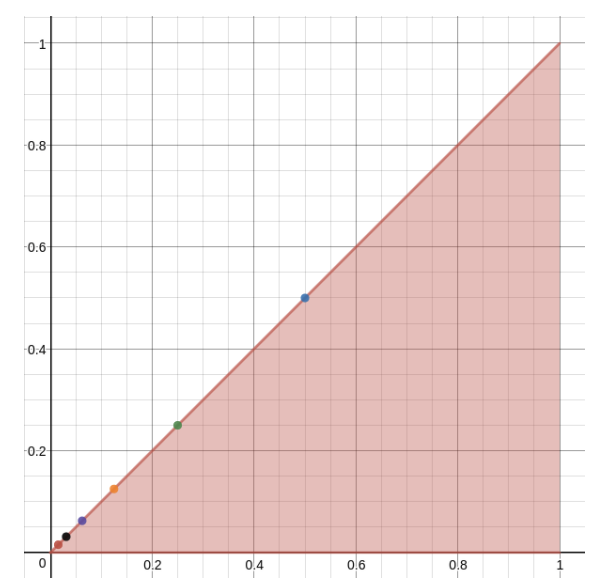

Figure 2.1: Points $\left(1 / 2^{k}, 1 / 2^{k}\right)$ of $\mathcal{L}_{H}, k \geq 1$.

Example 2.14. In general, computing the norm of a certain operator is really a difficult task. Nevertheless, if the operator is translation invariant, we have additional tools that might help us in that computation. 
Assume, for instance, that $g \in L^{1}$ and $g \geq 0$. The Fourier transform of $g$ is a continuous function vanishing at infinity by the Riemann-Lebesgue lemma (Lemma 1.13). In particular, $\|\widehat{g}\|_{\infty}$ is finite, and there exists $\xi_{0} \in \mathbb{R}^{n}$ so that $\|\widehat{g}\|_{\infty}=\widehat{g}\left(\xi_{0}\right)$. Moreover, we trivially have

$$
\|\widehat{g}\|_{\infty}=\sup _{\xi \in \mathbb{R}^{n}}\left|\int_{\mathbb{R}^{n}} g(x) e^{-2 \pi i \xi \cdot x} d x\right| \leq \int_{\mathbb{R}^{n}}|g(x)| d x=\|g\|_{1},
$$

and because $g \geq 0$,

$$
\|g\|_{1}=\int_{\mathbb{R}^{n}} g(x) d x=\widehat{g}(0) \leq\|\widehat{g}\|_{\infty},
$$

so we conclude that $\|\widehat{g}\|_{\infty}=\|g\|_{1}$.

With this, we are now going to compute the norm of the operator $A_{g}$ given by $A_{g} f=f * g$ as a bounded operator from $L^{p}$ to $L^{p}$ for several values of $p$. Let us start with the case $p=2$. Notice that for $f \in \mathcal{S}$,

$$
\|f * g\|_{2}=\|\widehat{f * g}\|_{2}=\|\widehat{f} \widehat{g}\|_{2} \leq\|\widehat{g}\|_{\infty}\|\widehat{f}\|_{2}=\|\widehat{g}\|_{\infty}\|f\|_{2}
$$

by Plancherel's theorem, and thus by density of $\mathcal{S}$ in $L^{2}$, the operator $A_{g}$ is bounded from $L^{2}$ to $L^{2}$ with $\left\|A_{g}\right\|_{2} \leq\|\widehat{g}\|_{\infty}$. On the other hand,

$$
\left\|A_{g}\right\|_{2}=\sup _{\substack{f \in L^{2} \\\|f\|_{2}=1}}\left\|A_{g} f\right\|_{2}=\sup _{\substack{f \in L^{2} \\\|f\|_{2}=1}}\|\widehat{f} \widehat{g}\|_{2}=\sup _{\substack{f \in L^{2} \\\|f\|_{2}=1}}\left(\int_{\mathbb{R}^{n}}|\widehat{f}(x)|^{2}|\widehat{g}(x)|^{2} d x\right)^{1 / 2}
$$

Thus, let $\left(f_{\varepsilon}\right)_{\varepsilon>0} \subset L^{2}$ be a sequence such that

$$
\widehat{f}_{\varepsilon}=\frac{1}{\left|B\left(\xi_{0}, \varepsilon\right)\right|^{1 / 2}} \chi_{B\left(\xi_{0}, \varepsilon\right)}
$$

Notice that such a sequence exists because the Fourier transform is a bijection on $L^{2}$ (Section 1.4). This sequence satisfies that

$$
\left\|f_{\varepsilon}\right\|_{2}=\left\|\widehat{f}_{\varepsilon}\right\|_{2}=1
$$

and because $\widehat{g}$ is continuous, the mean value theorem leads to

$$
\int_{\mathbb{R}^{n}}|\widehat{f}(x)|^{2}|\widehat{g}(x)|^{2} d x=\frac{1}{\left|B\left(\xi_{0}, \varepsilon\right)\right|} \int_{B\left(\xi_{0}, \varepsilon\right)}|\widehat{g}(x)|^{2} d x \stackrel{\varepsilon \rightarrow 0}{\longrightarrow}\left|\widehat{g}\left(\xi_{0}\right)\right|^{2}=\|\widehat{g}\|_{\infty}^{2},
$$

and so it must be $\left\|A_{g}\right\|_{2}=\|\widehat{g}\|_{\infty}$.

Consider now the case $p=1$. First, we simply have

$$
\left\|A_{g} f\right\|_{1}=\int_{\mathbb{R}^{n}}\left|\int_{\mathbb{R}^{n}} g(y) f(x-y) d y\right| d x \leq \int_{\mathbb{R}^{n}}|g(y)| \int_{\mathbb{R}^{n}}|f(x-y)| d x d y=\|g\|_{1}\|f\|_{1},
$$


so $A_{g}$ is strong type $(1,1)$ with $\left\|A_{g}\right\|_{1} \leq\|g\|_{1}$. Now,

$$
\left\|A_{g}\right\|_{1}=\sup _{\substack{f \in L^{1} \\\|f\|_{1}=1}}\left\|A_{g} f\right\|_{1}=\sup _{\substack{f \in L^{1} \\\|f\|_{1}=1}} \int_{\mathbb{R}^{n}}\left|\int_{\mathbb{R}^{n}} g(y) f(x-y) d y\right| d x,
$$

but $g(y) f(x-y) \leq g(y)|f(x-y)|$ for all $x, y \in \mathbb{R}^{n}$ because $g \geq 0$, and so by monotonicity of the integral, we can conclude that

$$
\left\|A_{g}\right\|_{1}=\sup _{\substack{f \in L^{1} \\\|f\|_{1}=1 \\ f \geq 0}} \int_{\mathbb{R}^{n}} \int_{\mathbb{R}^{n}} g(y) f(x-y) d y d x=\sup _{\substack{f \in L^{1} \\\|f\|_{1}=1 \\ f \geq 0}}\|g\|_{1}\|f\|_{1}=\|g\|_{1} .
$$

So far, thus, we have seen

$$
\begin{aligned}
& A_{g}: L^{1} \longrightarrow L^{1},\left\|A_{g}\right\|_{1}=\|g\|_{1}, \\
& A_{g}: L^{2} \longrightarrow L^{2},\left\|A_{g}\right\|_{2}=\|\widehat{g}\|_{\infty},
\end{aligned}
$$

and because $\|g\|_{1}=\|\widehat{g}\|_{\infty}$, we have $\left\|A_{g}\right\|_{1}=\left\|A_{g}\right\|_{2}$. Since $A_{g}$ is translation invariant, Theorem 2.9 ensures $A_{g}: L^{p} \rightarrow L^{p}$ for all $1 \leq p \leq 2$, and if we write $1 / p=1-\theta+\theta / 2$ for some $\theta \in(0,1)$, by $(2.4)$ we get

$$
\left\|A_{g}\right\|_{p} \leq\left\|A_{g}\right\|_{1}^{1-\theta}\left\|A_{g}\right\|_{2}^{\theta}=\|\widehat{g}\|_{\infty} .
$$

Finally, Corollary 2.12 states that $\left\|A_{g}\right\|_{2} \leq\left\|A_{g}\right\|_{p}$ for any $p$, so we also have directly $\|\widehat{g}\|_{\infty} \leq\left\|A_{g}\right\|_{p}$. All in all, and using the symmetry of the operator norm given again by Theorem 2.9, we conclude the following: for $g \in L^{1}$ with $g \geq 0$, the operator $A_{g}$ is strong type $(p, p)$ for any $1 \leq p \leq \infty$, and $\left\|A_{g}\right\|_{p}=\|\widehat{g}\|_{\infty}$.

In order to further study the spaces $L_{p}^{p}$, we shall jump into the spaces $M_{p}^{p}$ of their Fourier transforms. Notice that most of what we have said so far for $L_{p}^{p}$ is also valid for $M_{p}^{p}$. If $m \in S^{\prime}$ is a non-trivial tempered distribution, then

$$
\widehat{\mathcal{L}}_{m}=\left\{(s, t) \in \mathbb{R}^{2}: m \in M_{1 / s}^{1 / t}\right\}=\left\{(s, t) \in \mathbb{R}^{2}: \check{m} \in L_{1 / s}^{1 / t}\right\}=\mathcal{L}_{\check{m}},
$$

and also, $M_{p}^{p}(m)=L_{p}^{p}(\check{m})$ by definition. In particular, we have an analogous theorem to Theorem 2.9 for multipliers.

Theorem 2.15. Let $m \in S^{\prime}$ be a non-trivial tempered distribution. Then:

i) The set

$$
\widehat{\mathcal{L}}_{m}=\left\{(s, t) \in \mathbb{R}^{2}: m \in M_{1 / s}^{1 / t}\right\}
$$

is a convex subset of the triangle $0 \leq t \leq s \leq 1$ which is symmetric with respect to the line $s+t=1$.

ii) The function $M_{1 / s}^{1 / t}(m)$ is also symmetric in $\widehat{\mathcal{L}}_{m}$ with respect to $s+t=1$. 
iii) For any $(s, t),(r, w) \in \widehat{\mathcal{L}}_{m}$, and $\theta \in(0,1)$, it holds

$$
M_{p}^{q}(m) \leq\left[M_{1 / s}^{1 / t}(m)\right]^{1-\theta}\left[M_{1 / r}^{1 / w}(m)\right]^{\theta},
$$

where

$$
\begin{aligned}
& \frac{1}{p}=(1-\theta) s+\theta r \\
& \frac{1}{q}=(1-\theta) t+\theta w .
\end{aligned}
$$

As stated in Corollary 2.10 and Corollary 2.12, the spaces $M_{p}^{q}$ also satisfy $M_{p}^{q}=M_{q^{\prime}}^{p^{\prime}}$ for any $1 \leq p \leq q \leq \infty$, and if $q \leq p<2$ or $q \geq p>2$, then

$$
M_{q}^{q} \subset M_{p}^{p} \subset M_{2}^{2},
$$

with

$$
M_{2}^{2}(m) \leq M_{p}^{p}(m) \leq M_{q}^{q}(m), \forall m \in M_{q}^{q} .
$$

These two last facts follow directly from Theorem 2.15 with identical proofs to the ones of the referred corollaries.

Our advantage, now, is that $M_{2}^{2}$ can actually be characterized.

Theorem 2.16. With equality of norms,

$$
M_{2}^{2}=L^{\infty}
$$

Proof. The proof is basically the same as one of the arguments in Example 2.14. First, let $m \in M_{2}^{2}$. By definition, $\check{m} * f$ is an $L^{2}$ function for all $f \in L^{2}$, and so $m \widehat{f}$ is in $L^{2}$ for all $\widehat{f} \in L^{2}$ as well. In particular, by density of $\mathcal{S}$ in $L^{2}$, we conclude that $m$ is a locally square integrable function. Now, by Plancherel's theorem,

$$
\|m \widehat{f}\|_{2}=\|\check{m} * f\|_{2} \leq L_{2}^{2}(\check{m})\|f\|_{2}=M_{2}^{2}(m)\|\widehat{f}\|_{2},
$$

and because $\widehat{f}$ is an arbitrary $L^{2}$ function, we could just take, for each $\xi_{0} \in \mathbb{R}^{n}$, the sequence $\left(f_{\varepsilon}\right)_{\varepsilon>0} \subset L^{2}$ of functions of the form

$$
\widehat{f}_{\varepsilon}=\frac{1}{\left|B\left(\xi_{0}, \varepsilon\right)\right|^{1 / 2}} \chi_{B\left(\xi_{0}, \varepsilon\right)}
$$

which satisfy $\left\|f_{\varepsilon}\right\|_{2}=1$ for all $\varepsilon>0$, and which give us

$$
\left\|m f_{\varepsilon}\right\|_{2}=\left(\frac{1}{\left|B\left(\xi_{0}, \varepsilon\right)\right|} \int_{B\left(\xi_{0}, \varepsilon\right)}|m(x)|^{2} d x\right)^{1 / 2} \stackrel{\varepsilon \rightarrow 0}{\longrightarrow}\left|m\left(\xi_{0}\right)\right|
$$

for almost every chosen $\xi_{0}$, by Lebesgue differentiation theorem. Thus, $m \in L^{\infty}$ and $\|m\|_{\infty} \leq M_{2}^{2}(m)$. Conversely, if $m \in L^{\infty}$, then

$$
\|\check{m} * f\|_{2}=\|m \widehat{f}\|_{2} \leq\|m\|_{\infty}\|\widehat{f}\|_{2}=\|m\|_{\infty}\|f\|_{2}, \forall f \in L^{2},
$$

so $m \in M_{2}^{2}$ and $M_{2}^{2}(m)=L_{2}^{2}(\check{m}) \leq\|m\|_{\infty}$. All in all, $M_{2}^{2}=L^{\infty}$ and $M_{2}^{2}(m)=\|m\|_{\infty}$ for all $m \in M_{2}^{2}$. 
This theorem, combined with the fact that $M_{p}^{p} \subset M_{2}^{2}$ for all $p \neq 2$, is a remarkable result on what translation invariant operators really are. Recall that the Fourier transform of a distribution, even if it is given by the integral against an $L^{p}$ function, needs not be a function anymore. But, in virtue of this result, we are saying that Fourier transforms of any bounded translation invariant operator are actually given by bounded functions. This is why it is interesting and more convenient to consider multipliers instead of the spaces $L_{p}^{p}$. With this, we make the following definition.

Definition 2.17. A multiplier operator $T$ is an operator defined as

$$
\widehat{T f}=m \widehat{f}, \forall f \in \mathcal{S},
$$

where $m \in L^{\infty}$.

Because of what we have said, multiplier operators are a particular case of translation invariant operators, which are automatically bounded from $L^{2}$ to $L^{2}$. From now on, we will focus our attention on this type of operators.

With the notation of multipliers, it is very easy to see a couple of properties regarding invariance of their norms under certain manipulations such as translations and dilations.

Proposition 2.18. Let $m \in M_{p}^{p}, h \in \mathbb{R}^{n}$, and $\lambda>0$. Then,

i) $M_{p}^{p}\left(\tau_{h} m\right)=M_{p}^{p}(m)$.

ii) $M_{p}^{p}\left(\delta_{\lambda} m\right)=M_{p}^{p}(m)$, where $\delta_{\lambda}$ is the dilation operator, $\delta_{\lambda} m(\xi)=m(\xi / \lambda){ }^{2}$

Proof. We prove both properties using Proposition 1.14. For i), we have that

$$
\begin{aligned}
& M_{p}^{p}\left(\tau_{h} m\right)=\sup _{\substack{f \in \mathcal{S} \\
\|f\|_{p}=1}}\left\|\left(\widehat{f} \tau_{h} m\right)^{\vee}\right\|_{p}=\sup _{\substack{f \in \mathcal{S} \\
\|f\|_{p}=1}}\left\|\left(\tau_{h}\left(\tau_{-h} \widehat{f} m\right)\right)^{\vee}\right\|_{p} \\
& =\sup _{\substack{f \in \mathcal{S} \\
\|f\|_{p}=1}}\left\|e^{-2 \pi i h \cdot}\left(\widehat{e^{2 \pi i h \cdot}} f m\right)^{\vee}\right\|_{p}=\sup _{\substack{f \in \mathcal{S} \\
\|f\|_{p}=1}}\left\|(\widehat{f} m)^{\vee}\right\|_{p}=M_{p}^{p}(m),
\end{aligned}
$$

where the second last equality holds because $\left|e^{2 \pi i h \cdot} f\right|=|f|$. Similarly, for $\left.i i\right)$,

$$
\begin{aligned}
M_{p}^{p}\left(\delta_{\lambda} m\right)= & \sup _{\substack{f \in \mathcal{S} \\
\|f\|_{p}=1}}\left\|\left(\widehat{f} \delta_{\lambda} m\right)^{\vee}\right\|_{p}=\sup _{\substack{f \in \mathcal{S} \\
\|f\|_{p}=1}}\left\|\left(\delta_{\lambda}\left(\delta_{1 / \lambda} \widehat{f} m\right)\right)^{\vee}\right\|_{p} \\
= & \sup _{\substack{f \in \mathcal{S} \\
\|f\|_{p}=1}}\left\|\lambda^{n} \delta_{1 / \lambda}\left(\widehat{\lambda^{-n} \delta_{\lambda}} f m\right)^{\vee}\right\|_{p}=\sup _{\substack{f \in \mathcal{S} \\
\|f\|_{p}=1}}\left\|(\widehat{f} m)^{\vee}\right\|_{p}=M_{p}^{p}(m),
\end{aligned}
$$

and now, the second last equality is given by the fact that $\left\|\lambda^{n} \delta_{1 / \lambda} \varphi\right\|_{p}=\|\varphi\|_{p}$ for any $\lambda>0$ and any $\varphi \in L^{p}$.

\footnotetext{
${ }^{2}$ Notice that it makes sense to consider both $\tau_{h} m$ and $\delta_{\lambda} m$, because $m$ is a function thanks to the previous theorem.
} 
We now want to see another property of multipliers; namely, that they are closed under multiplication. This is very convenient, because it tells us that concatenating two multiplier operators $T_{1}$ and $T_{2}$ is the same considering the operator whose multiplier is the product of the multipliers of $T_{1}$ and $T_{2}$.

Proposition 2.19. Let $m_{1}, m_{2} \in M_{p}^{p}$, for $1<p<\infty$. Then, $m_{1} m_{2} \in M_{p}^{p}$ and

$$
M_{p}^{p}\left(m_{1} m_{2}\right) \leq M_{p}^{p}\left(m_{1}\right) M_{p}^{p}\left(m_{2}\right) .
$$

Proof. The case $p=2$ is trivial. On the other hand, by Theorem 2.15, it suffices to do the proof for $1<p<2$. Let $T_{1}$ be the multiplier operator associated to $m_{1}$, and $T_{2}$ the multiplier operator associated to $m_{2}$. By definition, we know that

$$
\widehat{T_{i} f}=\widehat{f} m_{i}, \forall f \in \mathcal{S} \text {. }
$$

We are going to prove now that

$$
\widehat{T_{i} f}=\widehat{f} m_{i}, \forall f \in L^{p} .
$$

Let us do the proof for $m_{1}$, since the same argument works as well for $m_{2}$. Consider the operator

$$
\text { Af }=\widehat{T_{1} f} \text {. }
$$

Since $m_{1} \in M_{p}^{p}$, we have that $T_{1} f \in L^{p}$ for any $f \in L^{p}$. Thus, by Hausdorff-Young's inequality (Theorem 1.17), we have that $\widehat{T_{1} f} \in L^{p^{\prime}}$ and that

$$
\left\|\widehat{T_{1} f}\right\|_{p^{\prime}} \leq\left\|T_{1} f\right\|_{p}
$$

In particular,

$$
\left\|\widehat{T_{1} f}\right\|_{p^{\prime}} \leq M_{p}^{p}\left(m_{1}\right)\|f\|_{p}
$$

so $A$ is strong type $\left(p, p^{\prime}\right)$. On the other hand, since $m_{1} \in L^{\infty}$, we have again, by Hausdorff-Young's inequality, that the operator

$$
B f=\widehat{f} m_{1},
$$

is strong type $\left(p, p^{\prime}\right)$ with

$$
\left\|\widehat{f} m_{1}\right\|_{p^{\prime}} \leq\left\|m_{1}\right\|\|f\|_{p}
$$

Therefore, we conclude that the operator $A-B$ is bounded from $L^{p}$ to $L^{p^{\prime}}$, but we have that $(A-B) f=0$ for any $f \in \mathcal{S}$. Since $\mathcal{S}$ is dense in $L^{p}$, we conclude that $(A-B) f=0$ for $f \in L^{p}$, and (2.9) follows.

With this equality, we can now prove what we are looking for. Take $f \in L^{p}$. Since $m_{2} \in M_{p}^{p}$, we have that $T_{2} f \in L^{p}$. Thus, we have that

$$
\widehat{T_{1} T_{2} f}=\widehat{T_{2} f} m_{1}=\widehat{f} m_{1} m_{2} .
$$

Of course, this equality is then also true for functions on $\mathcal{S}$, where we can write

$$
T_{1} T_{2} f=\left(\widehat{f} m_{1} m_{2}\right)^{\vee},
$$

and because $T_{1}$ and $T_{2}$ are strong type $(p, p)$, so is $T_{1} T_{2}$. Hence, in the end, $m_{1} m_{2}$ is an $M_{p}^{p}$ multiplier, which is the multiplier of the operator $T_{1} T_{2}$, and we have that $M_{p}^{p}\left(m_{1} m_{2}\right) \leq M_{p}^{p}\left(m_{1}\right) M_{p}^{p}\left(m_{2}\right)$ directly. 
Example 2.20 (Ball multipliers in $\mathbb{R}$ ). Let $T$ be the operator given by

$$
\widehat{T f}=\widehat{f} \chi_{(a, b)} .
$$

In view of Theorem 2.16, $\chi_{(a, b)}$ is indeed a multiplier, for it is a function in $L^{\infty}$, so $T$ is a translation invariant operator, bounded from $L^{2}$ to $L^{2}$. We want to prove that $T$ is actually bounded from $L^{p}$ to $L^{p}$ for any $1<p<\infty$, and to do so, we are going to use the boundedness of the Hilbert transform.

We had seen in Example 2.13 that if

$$
H(f)(x)=(h * f)(x)=\text { p.v. } \frac{1}{\pi} \int_{\mathbb{R}} \frac{f(y)}{x-y} d y
$$

is the Hilbert transform, where

$$
h(f)=\operatorname{p.v} \cdot \frac{1}{\pi} \int_{\mathbb{R}} \frac{f(y)}{y} d y,
$$

then $H$ is strong type $(p, p)$ for any $1<p<\infty$, and

$$
\widehat{H(f)}(\xi)=\widehat{h}(\xi) \widehat{f}(\xi)=-i \operatorname{sgn}(\xi) \widehat{f}(\xi)
$$

where $\operatorname{sgn}(\xi)$ is the sign function. That is, the multiplier associated to $H$ is $\widehat{h}(\xi)=$ $-i \operatorname{sgn}(\xi)$. Let us construct now the function $\chi_{(a, b)}$ in terms of $\widehat{h}$. Notice first that, simply,

$$
\chi_{(0,+\infty)}(\xi)=\frac{1}{2}(\operatorname{sgn}(\xi)+1),
$$

and with this, we have that

$$
\begin{aligned}
\chi_{(a, b)}(\xi) & =\chi_{(a,+\infty)}(\xi)-\chi_{(b,+\infty)}(\xi)=\chi_{(0,+\infty)}(\xi-a)-\chi_{(0,+\infty)}(\xi-b) \\
& =\frac{1}{2}(\operatorname{sgn}(\xi-a)-\operatorname{sgn}(\xi-b))=\frac{i}{2}(\widehat{h}(\xi-a)-\widehat{h}(\xi-b)) .
\end{aligned}
$$

Then, for any function $f$,

$$
\widehat{T f}(\xi)=\frac{i}{2}(\widehat{h}(\xi-a) \widehat{f}(\xi)-\widehat{h}(\xi-b) \widehat{f}(\xi)),
$$

but the term $\rho_{a}(\xi)=\widehat{h}(\xi-a) \widehat{f}(\xi)$ (and similarly, $\rho_{b}(\xi)=\widehat{h}(\xi-b) \widehat{f}(\xi)$ ) can be written as

$$
\begin{aligned}
\rho_{a}(\xi) & =\widehat{h}(\xi-a) \widehat{f}(\xi) \\
\rho_{a}(\xi+a) & =\widehat{h}(\xi) \widehat{f}(\xi+a) \\
\tau_{-a} \rho_{a}(\xi) & =\widehat{h}(\xi) \tau_{-a} \widehat{f}(\xi) \\
\rho_{a}(\xi) & =\tau_{a} \widehat{h}(\xi) \tau_{-a} \widehat{f}(\xi),
\end{aligned}
$$


so we conclude

$$
\begin{aligned}
& \widehat{T f}(\xi)=\frac{i}{2}\left(\tau_{a} \widehat{h}(\xi) \tau_{-a} \widehat{f}(\xi)-\tau_{b} \widehat{h}(\xi) \tau_{-b} \widehat{f}(\xi)\right)=\frac{i}{2}\left(\tau_{a} \widehat{h}(\xi) \widehat{e_{a} f}(\xi)-\tau_{b} \widehat{h}(\xi) \widehat{e_{b} f}(\xi)\right) \\
& \left.\left.=\frac{i}{2}\left(\tau_{a} \widehat{H\left(e_{a} f\right.}\right)(\xi)-\tau_{b} \widehat{H\left(e_{b} f\right.}\right)(\xi)\right)=\frac{i}{2}\left(\tau_{a} \widehat{H e_{a} f}(\xi)-\tau_{b} \widehat{H e_{b} f}(\xi)\right)
\end{aligned}
$$

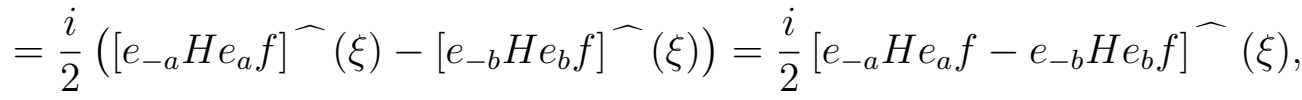

and thus $T$ is given by

$$
T=\frac{i}{2}\left[e_{-a} H e_{a}-e_{-b} H e_{b}\right]
$$

where $e_{s}$ is the operator defined as $\left(e_{s} f\right)(x)=e^{2 \pi i s x} f(x)$. Notice that $\left\|e_{s}\right\|_{p}=1$ for all $1<p<\infty$, so we conclude that $T$ is also bounded from $L^{p}$ to $L^{p}$ for any $1<p<\infty$, with norm $M_{p}^{p}\left(\chi_{(a, b)} \leq\|H\|_{p}\right.$. Actually, $M_{p}^{p}\left(\chi_{(a, b)}\right.$ is the same for any interval $(a, b)$, thanks to Proposition 2.18. Of course, the same argument, under little modifications, allow us to draw the same conclusion for characteristic functions of intervals of the form $[a, b),(a, b]$ or $[a, b]$.

One could think that a similar result for ball multipliers should hold in $\mathbb{R}^{n}$ for $n \geq 2$. It turns out that we do have boundedness for multipliers of the form $\chi_{Q}$, where $Q$ is a cube:

$$
Q=\left\{x \in \mathbb{R}^{n}: a_{i}<x_{i}<b_{i} \forall i=1, . ., n\right\},
$$

with $-\infty \leq a_{i}<b_{i} \leq \infty$. This is so because

$$
Q=\left(a_{1}, b_{1}\right) \times \cdots \times\left(a_{n}, b_{n}\right),
$$

which means that

$$
\chi_{Q}(\xi)=\prod_{i=1}^{n} \chi_{\left(a_{i}, b_{i}\right)}\left(\xi_{i}\right) .
$$

But we have just seen that every element of the former product is an $M_{p}^{p}$ multiplier for any $1<p<\infty$ and so Proposition 2.19 gives us that $\chi_{Q}$ is also an $M_{p}^{p}$ multiplier for $1<p<\infty$. On the other hand, the result is completely false for multipliers $\chi_{B}$, where $B$ is an euclidean ball in $\mathbb{R}^{n}, n \geq 2$. This is a non-trivial fact that will be discussed in Chapter 5.

From this last example, we can intuitively think that boundedness of certain multipliers in one dimension can extend to boundedness of multipliers in higher dimensions. We know face the reverse situation in a very specific way: is the restriction of a multiplier still a multiplier? The answer turns out to be yes, and the result is known as de Leeuw's theorem.

Theorem 2.21. Let $m(\xi)=m\left(\xi_{1}, \xi_{2}\right)$ be an $M_{p}^{p}\left(\mathbb{R}^{n+m}\right)$ multiplier, for some $1 \leq p \leq \infty$. Then, for almost any $\xi_{1} \in \mathbb{R}^{n}$, the function $m_{\xi_{1}}$ given by $m_{\xi_{1}}\left(\xi_{1}\right)=m\left(\xi_{1}, \xi_{2}\right)$ is an $M_{p}^{p}\left(\mathbb{R}^{m}\right)$ multiplier. 
Proof. If $m \in M_{p}^{p}\left(\mathbb{R}^{n+m}\right)$, it means that for any $\phi \in \mathcal{S}\left(\mathbb{R}^{n+m}\right)$, we have

$$
\left\|(m \widehat{\phi})^{\vee}\right\|_{p} \leq M_{p}^{p}(m)\|\phi\|_{p}
$$

Notice that

$$
\begin{aligned}
&\left\|(m \widehat{\phi})^{\vee}\right\|_{p}=\sup _{\substack{\psi \in \mathcal{S}\left(\mathbb{R}^{n+m}\right) \\
\|\psi\|_{p^{\prime}}=1}}\left|\int_{\mathbb{R}^{n+m}}(m \widehat{\phi})^{\vee}(x) \psi(x) d x\right| \\
&=\sup _{\substack{\psi \in \mathcal{S}\left(\mathbb{R}^{n+m}\right) \\
\|\psi\|_{p^{\prime}}=1}}\left|\int_{\mathbb{R}^{n+m}} \int_{\mathbb{R}^{n+m}} m(\xi) \widehat{\phi}(\xi) e^{2 \pi i \xi \cdot x} d \xi \psi(x) d x\right| \\
&=\sup _{\substack{\psi \mathcal{S}\left(\mathbb{R}^{n+m}\right) \\
\|\psi\|_{p^{\prime}}=1}}\left|\int_{\mathbb{R}^{n+m}} m(\xi) \widehat{\phi}(\xi) \int_{\mathbb{R}^{n+m}} \psi(x) e^{2 \pi i \xi \cdot x} d x d \xi\right| \\
&=\sup _{\substack{\psi \in \mathcal{S}\left(\mathbb{R}^{n+m}\right) \\
\|\psi\|_{p^{\prime}}=1}}\left|\int_{\mathbb{R}^{n+m}} m(\xi) \widehat{\phi}(\xi) \widehat{\psi}(-\xi) d \xi\right|, \\
&
\end{aligned}
$$

so we have that $m \in M_{p}^{p}\left(\mathbb{R}^{n+m}\right)$ if and only if

$$
\left|\int_{\mathbb{R}^{n+m}} m(\xi) \widehat{\phi}(\xi) \widehat{\psi}(\xi) d \xi\right| \leq M_{p}^{p}(m)\|\phi\|_{p}\|\psi\|_{p^{\prime}}
$$

for any $\phi, \psi \in \mathcal{S}\left(\mathbb{R}^{n+m}\right)$. With this, let $f, g \in \mathcal{S}\left(\mathbb{R}^{m}\right)$, and $a, b \in \mathcal{S}\left(\mathbb{R}^{n}\right)$, and define $\phi(x)=a\left(x_{1}\right) f\left(x_{2}\right)$ and $\psi(x)=b\left(x_{1}\right) g\left(x_{2}\right)$. It is clear that $\widehat{\phi}(\xi)=\widehat{a}\left(\xi_{1}\right) \widehat{f}\left(\xi_{2}\right)$ and $\widehat{\psi}(\xi)=\widehat{b}\left(\xi_{1}\right) \widehat{g}\left(\xi_{2}\right)$, as well as $\|\phi\|_{p}=\|a\|_{p}\|f\|_{p}$ and $\|\psi\|_{p^{\prime}}=\|b\|_{p^{\prime}}\|g\|_{p^{\prime}}$, so we can write

$$
\left|\int_{\mathbb{R}^{n}}\left(\int_{\mathbb{R}^{m}} m(\xi) \widehat{f}\left(\xi_{2}\right) \widehat{g}\left(\xi_{2}\right) d \xi_{2}\right) \widehat{a}\left(\xi_{1}\right) \widehat{b}\left(\xi_{1}\right) d \xi_{1}\right| \leq M_{p}^{p}(m)\|f\|_{p}\|g\|_{p^{\prime}}\|a\|_{p}\|b\|_{p^{\prime}},
$$

which is saying, according to the previous characterization, that the multiplier

$$
m^{\prime}\left(\xi_{1}\right)=\int_{\mathbb{R}^{m}} m(\xi) \widehat{f}\left(\xi_{2}\right) \widehat{g}\left(\xi_{2}\right) d \xi_{2}=\int_{\mathbb{R}^{m}} m_{\xi_{1}}\left(\xi_{2}\right) \widehat{f}\left(\xi_{2}\right) \widehat{g}\left(\xi_{2}\right) d \xi_{2}
$$

is bounded from $L^{p}\left(\mathbb{R}^{n}\right)$ to itself with norm at most

$$
M_{p}^{p}\left(m^{\prime}\right) \leq M_{p}^{p}(m)\|f\|_{p}\|g\|_{p^{\prime}} .
$$

But because $M_{2}^{2}\left(m^{\prime}\right) \leq M_{p}^{p}\left(m^{\prime}\right)$ and $M_{2}^{2}\left(m^{\prime}\right)=\left\|m^{\prime}\right\|_{\infty}$ in virtue of Theorem 2.16, we have that

$$
\left|\int_{\mathbb{R}^{m}} m_{\xi_{1}}\left(\xi_{2}\right) \widehat{f}\left(\xi_{2}\right) \widehat{g}\left(\xi_{2}\right) d \xi_{2}\right| \leq M_{p}^{p}(m)\|f\|_{p}\|g\|_{p^{\prime}}
$$

which means that $m_{\xi_{1}} \in M_{p}^{p}\left(\mathbb{R}^{m}\right)$, as we wanted. 


\section{Chapter 3}

\section{Littlewood-Paley theory}

We now know that every multiplier must be a bounded function. But of course, we still do not know, for $m \in L^{\infty}$, for which $1 \leq p \leq \infty$ we have that $m \in M_{p}^{p}$. This question, though, is still widely open, but there are a couple of results, namely, Mihlin-Hörmander's theorem and Marcinkiewicz's theorem, which are sufficient conditions on bounded functions in order to ensure that their associated translation invariant operators are bounded for a large range of values of $p$. We will focus on these results in the following chapter, for in order to prove them, we need first to introduce a set of results known as Littlewood-Paley theory, which is a way of extending results in $L^{2}$ to functions in $L^{p}$ regarding the decomposition of a function into a sum of functions of localized frequencies.

\subsection{Introduction}

Let us start with some heuristic arguments that will justify the spirit of LittlewoodPaley theory. Let $f$ and $g$ be functions in $L^{2}$. Recall that two such functions are called orthogonal if

$$
\langle f, g\rangle:=\int_{\mathbb{R}^{n}} f(x) g(x) d x=0 .
$$

Another way to look at orthogonality is the following: $f$ and $g$ are orthogonal functions if and only if they satisfy the Pythagorean theorem:

$$
\|f+g\|_{2}^{2}=\|f\|_{2}^{2}+\|g\|_{2}^{2}
$$

This definition of orthogonality has a nice advantage with respect to the first one, namely, that it can be extended to an arbitrary family of functions: if $\left(f_{k}\right)_{k \in K}$ is a collection of $L^{2}$ functions indexed by some set $K$, then we can say that the family is orthogonal if

$$
\left\|\sum_{k \in K} f_{k}\right\|_{2}^{2}=\sum_{k \in K}\left\|f_{k}\right\|_{2}^{2},
$$

whenever this former expression makes sense. Notice that this is the case, for instance, if the family $\left(f_{k}\right)_{k \in K}$ consists on functions such that their Fourier transforms 
$\left(\widehat{f}_{k}\right)_{k \in K}$ have pairwise disjoint supports, and $\sum_{k \in K}\left\|\widehat{f}_{k}\right\|_{2}^{2}<+\infty$. Indeed, in this setting, the expression

$$
\sum_{k \in K} \widehat{f}_{k}
$$

is trivially a well defined function, which is in $L^{2}$ because

$$
\left\|\sum_{k \in K} \widehat{f}_{k}\right\|_{2}^{2}=\sum_{k \in K}\left\|\widehat{f}_{k}\right\|_{2}^{2}<+\infty
$$

and so by Plancherel's theorem,

$$
\sum_{k \in K}\left\|f_{k}\right\|_{2}^{2}=\sum_{k \in K}\left\|\widehat{f}_{k}\right\|_{2}^{2}=\left\|\sum_{k \in K} \widehat{f}_{k}\right\|_{2}^{2}=\left\|\widehat{\sum_{k \in K} f_{k}}\right\|_{2}^{2}=\left\|\sum_{k \in K} f_{k}\right\|_{2}^{2} .
$$

This inspires us to consider a similar definition for a general $L^{p}$ space with $p \neq 2$. That is, we could say that a family $\left(f_{k}\right)_{k \in K}$ of functions in $L^{p}$ is orthogonal if their Fourier transforms have disjoint supports. In this sense, we can think about an orthogonal decomposition of an $L^{p}$ function. Indeed, take for each $k \in \mathbb{Z}$ the functions

$$
\psi_{2^{-k}}(x)=2^{n k} \psi\left(2^{k} x\right), \forall x \in \mathbb{R}^{n},
$$

where $\widehat{\psi}$ is the characteristic function of the annulus $\left\{x \in \mathbb{R}^{n}: 1<|x| \leq 2\right\}$. Notice, then, that

$$
\widehat{\psi_{2^{-k}}}(\xi)=\widehat{\psi}\left(2^{-k} \xi\right)
$$

so $\widehat{\psi_{2^{-k}}}$ is the characteristic function of the annulus $\left\{x \in \mathbb{R}^{n}: 2^{k}<|x| \leq 2^{k+1}\right\}$. The functions $\widehat{\psi_{2^{-k}}}$ have disjoint supports, so if $f \in L^{p}$, and $f$ has a Fourier transform $\widehat{f}$, we can trivially write

$$
\widehat{f}(\xi)=\sum_{k \in \mathbb{Z}} \widehat{\psi_{2^{-k}}}(\xi) \widehat{f}(\xi),
$$

for almost every $\xi \in \mathbb{R}^{n}$, and so, at least formally, we have that

$$
f=\sum_{k \in \mathbb{Z}} \psi_{2^{-k}} * f
$$

The idea of this decomposition is to express the function $f$ as a sum of functions $\psi_{2^{-k}} * f$ such that they only contain the frequencies of $f$ which are between $2^{k}$ and $2^{k+1}$; that is, as a sum of orthogonal components of $f$. This is an interesting way of decomposing $f$, and if $p=2$, we would have by (3.1) that

$$
\|f\|_{2}^{2}=\left\|\sum_{k \in \mathbb{Z}} \psi_{2^{-k}} * f\right\|_{2}^{2}=\sum_{k \in \mathbb{Z}}\left\|\psi_{2^{-k}} * f\right\|_{2}^{2},
$$

which is a direct relation between the size of the function and the size of each of its orthogonal components. For $p \neq 2$, though, we have to be careful with this definition of orthogonality, because we might as well expect the analogous expression

$$
\|f\|_{p}^{p} \approx \sum_{k \in \mathbb{Z}}\left\|\psi_{2^{-k}} * f\right\|_{p}^{p}
$$

to hold in this situation, and it turns out that this might not be really the case. 
Example 3.1. If $p>2$, we shall see that

$$
\left\|\sum_{k \in K} f_{k}\right\|_{p}^{p} \leq C_{p} \sum_{k \in K}\left\|f_{k}\right\|_{p}^{p}
$$

cannot be true in general, for any constant $C_{p}>0$. Let $\phi \in \mathcal{S}$ be such that $\widehat{\phi}$ is positive and with support in the set $\{\xi \in \mathbb{R}:|\xi|<1 / 4\}$. Without loss of generality, $\phi$ does not vanish in a neighbourhood of 0 , because $\widehat{\tau_{x} \phi}(\xi)=e^{-2 \pi i \xi x} \widehat{\phi}(\xi)$ for any $x \in \mathbb{R}$, so we can translate the function $\phi$, if necessary, without altering the support of its Fourier transform. Now, for $k \in \mathbb{N}$, set

$$
f_{k}(x)=e^{2 \pi i k x} \phi(x)
$$

By the properties of the Fourier transform, we trivially have $\widehat{f}_{k}(\xi)=\widehat{\phi}(\xi-k)$, and so the family $\left(\widehat{f}_{k}\right)_{k \in \mathbb{N}}$ have pairwise disjoint supports. Now, it is immediate that, for $N \in \mathbb{N}$,

$$
\sum_{k=0}^{N}\left\|f_{k}\right\|_{p}^{p}=\sum_{k=0}^{N} \int_{\mathbb{R}}\left|e^{2 \pi i k x} \phi(x)\right|^{p} d x=(N+1)\|\phi\|_{p}^{p} .
$$

On the other hand,

$$
\left\|\sum_{k=0}^{N} f_{k}\right\|_{p}^{p}=\int_{\mathbb{R}}\left|\sum_{k=0}^{N} e^{2 \pi i k x}\right|^{p}|\phi(x)|^{p} d x=\int_{\mathbb{R}}\left|\frac{e^{2 \pi i(N+1) x}-1}{e^{2 \pi i x}-1}\right|^{p}|\phi(x)|^{p} d x,
$$

and

$$
\begin{aligned}
\left|\frac{e^{2 \pi i(N+1) x}-1}{e^{2 \pi i x}-1}\right|^{2} & =\frac{1-\cos (2 \pi(N+1) x)}{1-\cos (2 \pi x)} \geq \frac{1-\cos (2 \pi(N+1) x)}{4 \pi^{2} x^{2}} \\
& =\frac{4 \pi^{2}(N+1)^{2} x^{2} \varphi(2 \pi(N+1) x)}{4 \pi^{2} x^{2}} \\
& =(N+1)^{2} \varphi(2 \pi(N+1) x)
\end{aligned}
$$

where

$$
\varphi(x)=\sum_{k=1}^{\infty} \frac{(-1)^{k+1} x^{2 k-2}}{(2 k) !}=\frac{1-\cos (x)}{x^{2}}, \forall x \in \mathbb{R} .
$$

Now, notice that the function $\varphi$ is continuous, non-negative, has a local maximum at $x=0$ with $\varphi(0)=1 / 2$, and has the same set of zeros as $1-\cos (x)$ except from this maximum; that is,

$$
\mathcal{Z}(\varphi)=\{x=2 k \pi: k \in \mathbb{Z} \backslash\{0\}\}
$$

Thus, if we take, for instance, $\varepsilon=1 /(2(N+1))$, we get

$$
C^{2 / p}:=\inf _{x \in B(0, \varepsilon)} \varphi(2 \pi(N+1) x)=\varphi(\pi)>0,
$$


and so

$$
\begin{aligned}
\int_{\mathbb{R}}\left|\frac{e^{2 \pi i(N+1) x}-1}{e^{2 \pi i x}-1}\right|^{p}|\phi(x)|^{p} d x & \geq C(N+1)^{p} \int_{B(0, \varepsilon)}|\phi(x)|^{p} d x \\
& =C(N+1)^{p}|B(0, \varepsilon)|\left|\phi\left(x_{0}\right)\right|^{p} \\
& =C(N+1)^{p-1}\left|\phi\left(x_{0}\right)\right|^{p}
\end{aligned}
$$

where the second last equality is given by the mean value theorem, and $x_{0} \in B(0, \varepsilon)$. Hence, let $N_{0}$ be large enough so that

$$
\inf _{|x|<1 /(2(N+1))}|\phi(x)|^{p} \geq C_{\phi}>0
$$

for any $N \geq N_{0}$, which is possible because $\phi$ does not vanish in a neighbourhood of the origin. For such $N$, we conclude that

$$
\left|\frac{e^{2 \pi i(N+1) x}-1}{e^{2 \pi i x}-1}\right|^{2} \geq C C_{\phi}(N+1)^{p-1},
$$

and this finally means that we cannot have an inequality of the form

$$
\left\|\sum_{k \in K} f_{k}\right\|_{p}^{p} \leq C_{p} \sum_{k \in K}\left\|f_{k}\right\|_{p}^{p}
$$

because it would mean that

$$
C C_{\phi}(N+1)^{p-2} \leq C_{p}\|\phi\|_{p}^{p}
$$

for every $N \geq N_{0}$, and this is not possible since $p>2$.

For $p<2$, the reverse inequality

$$
\left\|\sum_{k \in K} f_{k}\right\|_{p}^{p} \geq C_{p} \sum_{k \in K}\left\|f_{k}\right\|_{p}^{p}
$$

is the one that is not fulfilled in general for any constant $C_{p}>0$ (see [5, Example 6.1.9] for a construction in this case). With this, we want to illustrate that we have to look for different estimates when considering orthogonal decompositions of functions in $L^{p}$ similar to (3.2). For instance, notice that, at least in $L^{2}$, one has for our choice of $\psi$ that

$$
\|f\|_{2}=\left(\sum_{k \in \mathbb{Z}}\left\|\psi_{2^{-k}} * f\right\|_{2}^{2}\right)^{1 / 2}=\left\|\left(\sum_{k \in \mathbb{Z}}\left|\psi_{2^{-k}} * f\right|^{2}\right)^{1 / 2}\right\|_{2},
$$

by the monotone convergence theorem. But now, the object $\left(\sum_{k \in \mathbb{Z}}\left|\psi_{2^{-k}} * f\right|^{2}\right)^{1 / 2}$ still contains the information about the size of each orthogonal component of the decomposition of $f$ without depending on the particular choice of the $L^{p}$ space, and so we might want to look for $L^{p}$ estimates of this object itself. This is going to be the key ingredient of Littlewood-Paley theory. 


\subsection{Littlewood-Paley operators and orthogonal de- composition}

Let us begin giving the definition of the so called Littlewood-Paley operators, which generalize the kind of decompositions that we have just examined.

Definition 3.2. Let $\psi \in L^{1}$, and $k \in \mathbb{Z}$. The Littlewood-Paley operator $\Delta_{k}$ associated with $\psi$ is the operator given by

$$
\Delta_{k}(f)=f * \psi_{2^{-k}},
$$

where $\psi_{2^{-k}}(x)=2^{n k} \psi\left(2^{k} x\right)$ for all $x \in \mathbb{R}^{n}$.

As said, because of the properties of the Fourier transform, it holds that

$$
\widehat{\psi_{2^{-k}}}(\xi)=\widehat{\psi}\left(2^{-k} \xi\right), \forall \xi \in \mathbb{R}^{n}
$$

Definition 3.3. The square function associated with the Littlewood-Paley operators $\Delta_{k}, k \in \mathbb{Z}$, is the map $s_{\psi}$ defined as

$$
s_{\psi}(f)=\left(\sum_{k \in \mathbb{Z}}\left|\Delta_{k}(f)\right|^{2}\right)^{1 / 2} .
$$

We now give a weak version of the main result of this theory, called LittlewoodPaley theorem, which will be enough for our purposes. The theorem essentially gives us some insight on the estimates that we were looking for in our initial discussion. For a stronger version of the theorem, see [5, Theorem 6.1.2].

Theorem 3.4 (Littlewood-Paley theorem). Let $\psi$ be a function in $\mathcal{S}$, such that

$$
\int_{\mathbb{R}^{n}} \psi(x) d x=0
$$

Then:

i) The square function $s_{\psi}$ is strong type $(p, p)$ for $1<p<\infty$, and weak type $(1,1)$.

ii) Conversely, if $\psi$ also satisfies that

$$
\sum_{k \in \mathbb{Z}}\left|\widehat{\psi_{2^{-k}}}(\xi)\right|^{2}=1, \forall \xi \in \mathbb{R}^{n} \backslash\{0\}
$$

then

$$
\|f\|_{p} \lesssim\left\|\left(\sum_{k \in \mathbb{Z}}\left|\Delta_{k}(f)\right|^{2}\right)^{1 / 2}\right\|_{p},
$$

for all $f \in L^{p}$, with $1<p<\infty$. In particular, in this situation,

$$
\|f\|_{p} \approx\left\|\left(\sum_{k \in \mathbb{Z}}\left|\Delta_{k}(f)\right|^{2}\right)^{1 / 2}\right\|_{p}, \forall f \in L^{p} .
$$


Proof. Let us begin proving the first part of the theorem. Notice that saying that $s_{\psi}$ is strong type $(p, p)$ for $1<p<\infty$ and weak type $(1,1)$ is equivalent to saying that the map

$$
\vec{T}(f)=\left\{\Delta_{k}(f)\right\}_{k \in \mathbb{Z}}
$$

is bounded from $L^{p}$ to $L^{p}\left(\mathbb{R}^{n}, \ell^{2}\right)$ and also from $L^{1}$ to $L^{1, \infty}\left(\mathbb{R}^{n}, \ell^{2}\right)$. Thus, we want to apply Theorem 1.10 to prove such boundedness estimates, taking $X=\mathbb{R}$ and $Y=\ell^{2}$ in the statement.

We are going to start, then, by proving that $\vec{T}$ is bounded from $L^{2}$ to $L^{2}\left(\mathbb{R}^{n}, \ell^{2}\right)$; that is, that $s_{\psi}$ is strong type $(2,2)$. First, notice that

$$
\begin{aligned}
\left\|\left(\sum_{k \in \mathbb{Z}}\left|\widehat{\Delta_{k}(f)}\right|^{2}\right)^{1 / 2}\right\|_{2} & =\left(\int_{\mathbb{R}^{n}} \sum_{k \in \mathbb{Z}}\left|\widehat{\Delta_{k}(f)}(\xi)\right|^{2} d \xi\right)^{1 / 2} \\
& =\left(\sum_{k \in \mathbb{Z}} \int_{\mathbb{R}^{n}}\left|\widehat{\Delta_{k}(f)}(\xi)\right|^{2} d \xi\right)^{1 / 2}
\end{aligned}
$$

where the last equality is given by the monotone convergence theorem, and so using Plancherel's theorem,

$$
\begin{aligned}
\left(\sum_{k \in \mathbb{Z}} \int_{\mathbb{R}^{n}}\left|\widehat{\Delta_{k}(f)}(\xi)\right|^{2} d \xi\right)^{1 / 2} & =\left(\sum_{k \in \mathbb{Z}}\left\|\widehat{\Delta_{k}(f)}\right\|_{2}^{2}\right)^{1 / 2}=\left(\sum_{k \in \mathbb{Z}}\left\|\Delta_{k}(f)\right\|_{2}^{2}\right)^{1 / 2} \\
& =\left(\sum_{k \in \mathbb{Z}} \int_{\mathbb{R}^{n}}\left|\Delta_{k}(f)\right|^{2} d x\right)^{1 / 2}=\left(\int_{\mathbb{R}^{n}} \sum_{k \in \mathbb{Z}}\left|\Delta_{k}(f)\right|^{2} d x\right)^{1 / 2} \\
& =\left\|\left(\sum_{k \in \mathbb{Z}}\left|\Delta_{k}(f)\right|^{2}\right)^{1 / 2}\right\|_{2}
\end{aligned}
$$

using again the monotone convergence theorem. Thus, let us find estimates for the Fourier transform of $\psi$. Since $\psi \in \mathcal{S}$, and $\partial_{x_{i}} \psi=\partial \psi / \partial x_{i}$ for each $i=1, \ldots, n$, then

$$
\begin{aligned}
|\psi(x)|+|\nabla \psi(x)| & =|\psi(x)|+\left(\left(\partial_{x_{1}} \psi(x)\right)^{2}+\cdots+\left(\partial_{x_{n}} \psi(x)\right)^{2}\right)^{1 / 2} \\
& \leq|\psi(x)|+\left|\partial_{x_{1}} \psi(x)\right|+\cdots+\left|\partial_{x_{n}} \psi(x)\right| \\
& \leq \frac{1}{\left(1+|x|^{2}\right)^{n+1}} n p_{n+1}(\psi) \\
& \leq \frac{n p_{n+1}(\psi)}{2^{n+1}(\sqrt{2}-1)^{n+1}} \frac{1}{(1+|x|)^{n+1}}:=B_{\psi, n} \frac{1}{(1+|x|)^{n+1}}
\end{aligned}
$$

for $\left(1+|x|^{2}\right) \geq 2(\sqrt{2}-1)(1+|x|)$ because the function $g(t)=\left(1+t^{2}\right) /(1+t)$ is differentiable in $(0,+\infty)$, and it attains a minimum value $2(\sqrt{2}-1)$ at $t=\sqrt{2}-1$. Now, using that $\psi$ has mean value zero, we have that

$$
\widehat{\psi}(\xi)=\int_{\mathbb{R}^{n}} e^{-2 \pi i \xi \cdot x} \psi(x) d x=\int_{\mathbb{R}^{n}}\left(e^{-2 \pi i \xi \cdot x}-1\right) \psi(x) d x,
$$


and so

$$
|\widehat{\psi}(\xi)| \leq \int_{\mathbb{R}^{n}}\left|e^{-2 \pi \xi \cdot x}-1\right||\psi(x)| d x=\int_{\mathbb{R}^{n}} \sqrt{2}(1-\cos (2 \pi \xi \cdot x))^{1 / 2}|\psi(x)| d x,
$$

but notice that we can write

$$
1-\cos (2 \pi \xi \cdot x) \leq 2 \pi|\xi \cdot x| \leq 2 \pi|\xi||x|,
$$

because the functions $h_{1}(t)=1-\cos (2 \pi t)$ and $h_{2}(t)=2 \pi|t|$ are even functions, which are continuous functions in $\mathbb{R}$ and differentiable in $\mathbb{R} \backslash\{0\}$, and satisfy $h_{1}(0)=h_{2}(0)$ and $h_{1}^{\prime}(t)=2 \pi \sin (2 \pi t) \leq 2 \pi=h_{2}^{\prime}(t)$ for $t>0$, and with all this, we get the estimate

$$
|\widehat{\psi}(\xi)| \leq \sqrt{4 \pi}|\xi|^{1 / 2} B_{\psi, n} \int_{\mathbb{R}^{n}} \frac{|x|^{1 / 2}}{(1+|x|)^{n+1}} d x:=\sqrt{4 \pi} B_{\psi, n} C_{1, n}|\xi|^{1 / 2}
$$

On the other hand, given $\xi \in \mathbb{R}^{n}$, let $\xi_{k}$ be such that $\left|\xi_{k}\right|=\|\xi\|_{\infty}$, and integrate by parts in the definition of $\widehat{\psi}$ with respect to this component, so that we get

$$
\widehat{\psi}(\xi)=\int_{\mathbb{R}^{n}} e^{-2 \pi i \xi \cdot x} \psi(x) d x=-\int_{\mathbb{R}^{n}} \frac{1}{-2 \pi i \xi_{k}} e^{-2 \pi i \xi \cdot x} \partial_{x_{k}} \psi(x) d x,
$$

and so using that $\left|\xi_{k}\right|=\sqrt{n\left|\xi_{k}\right|^{2}} / \sqrt{n} \geq|\xi| / \sqrt{n}$, we obtain another estimate

$$
|\widehat{\psi}(\xi)| \leq \frac{\sqrt{n}}{2 \pi|\xi|} \int_{\mathbb{R}^{n}}\left|\partial_{x_{k}} \psi(x)\right| d x \leq \frac{\sqrt{n}}{2 \pi|\xi|} \int_{\mathbb{R}^{n}} \frac{B_{\psi, n}}{(1+|x|)^{n+1}} d x:=\frac{B_{\psi, n} C_{2, n}}{2 \pi} \frac{1}{|\xi|} .
$$

Now, for $\xi \in \mathbb{R}^{n}$, we split the sum

$$
\sum_{k \in \mathbb{Z}}\left|\widehat{\psi_{2^{-k}}}(\xi)\right|^{2}=\sum_{k \in \mathbb{Z}}\left|\widehat{\psi}\left(2^{-k} \xi\right)\right|^{2}=\sum_{k \leq k_{0}}\left|\widehat{\psi}\left(2^{-k} \xi\right)\right|^{2}+\sum_{k \geq k_{0}+1}\left|\widehat{\psi}\left(2^{-k} \xi\right)\right|^{2}
$$

where $k_{0}$ is such that $2^{-k}|\xi| \geq 1$ if $k \leq k_{0}$ and $2^{-k}|\xi| \leq 1$ if $k \geq k_{0}+1$. Using (3.5) in the first part and (3.4) in the second one yields to

$$
\begin{aligned}
\sum_{k \in \mathbb{Z}}\left|\widehat{\psi_{2^{-k}}}(\xi)\right|^{2} & \leq \sum_{k \leq k_{0}} \frac{B_{\psi, n}^{2} C_{2, n}^{2}}{4 \pi^{2}} \frac{1}{\left|2^{-k} \xi\right|^{2}}+\sum_{k \geq k_{0}+1} 4 \pi B_{\psi, n}^{2} C_{1, n}^{2}\left|2^{-k} \xi\right| \\
& \leq \frac{B_{\psi, n}^{2} C_{2, n}^{2}}{4 \pi^{2}} \sum_{k \leq 0} \frac{1}{4^{k}}+4 \pi B_{\psi, n}^{2} C_{1, n}^{2} \sum_{k \geq 0} \frac{1}{2^{k}} \\
& =B_{\psi, n}^{2}\left(\frac{C_{2, n}^{2}}{3 \pi^{2}}+8 \pi C_{1, n}^{2}\right):=B_{\psi, n}^{2} C_{n}^{2}
\end{aligned}
$$

All in all, we conclude that

$$
\begin{aligned}
\left\|\left(\sum_{k \in \mathbb{Z}}\left|\Delta_{k}(f)\right|^{2}\right)^{1 / 2}\right\|_{2} & =\left\|\left(\sum_{k \in \mathbb{Z}}\left|\widehat{\Delta_{k}(f)}\right|^{2}\right)^{1 / 2}\right\|_{2}=\left\|\left(|\widehat{f}|^{2} \sum_{k \in \mathbb{Z}}\left|\widehat{\psi_{2^{-k}}}\right|^{2}\right)^{1 / 2}\right\|_{2} \\
& \leq B_{\psi, n} C_{n}\|\widehat{f}\|_{2}=B_{\psi, n} C_{n}\|f\|_{2},
\end{aligned}
$$

and so $s_{\psi}$ is strong type $(2,2)$. 
Now, let us see the result for the rest of values of $p$, using Theorem 1.10 as we have said at the beginning. With what we have just seen, we already have one of the hypothesis of the theorem, namely, that $\vec{T}$ is bounded from $L^{2}$ to $L^{2}\left(\mathbb{R}^{n}, \ell^{2}\right)$. Also, notice that the operator $\vec{T}$ defined in (3.3) is given by

$$
\begin{aligned}
\vec{T}(f)(x) & =\left\{\Delta_{k}(f)(x)\right\}_{k \in \mathbb{Z}}=\left\{\left(\psi_{2^{-k}} * f\right)(x)\right\}_{k \in \mathbb{Z}} \\
& =\left\{\int_{\mathbb{R}^{n}} \psi_{2^{-k}}(y) f(x-y) d y\right\}_{k \in \mathbb{Z}}=\int_{\mathbb{R}^{n}} \vec{K}(y)(f(x-y)) d y,
\end{aligned}
$$

where $\vec{K}: \mathbb{R}^{n} \rightarrow L\left(\mathbb{R}, \ell^{2}\right)$ is the map defined as

$$
\vec{K}(y)(a)=\left\{\psi_{2^{-k}}(y) a\right\}_{k \in \mathbb{Z}} .
$$

Thus, it remains to see that for some $A>0$ and $\vec{K}_{0} \in L\left(\mathbb{R}, \ell^{2}\right)$ we have:

i) $\|\vec{K}(x)\|_{\mathbb{R} \rightarrow \ell^{2}} \leq A|x|^{-n}$, where $\|\cdot\|_{\mathbb{R} \rightarrow \ell^{2}}$ denotes the operator norm in $L\left(\mathbb{R}, \ell^{2}\right)$.

ii) $\sup _{y \in \mathbb{R}^{n} \backslash\{0\}} \int_{|x| \geq 2|y|}\|\vec{K}(x-y)-\vec{K}(x)\|_{\mathbb{R} \rightarrow \ell^{2}} d x \leq A$.

iii) $\lim _{\varepsilon \rightarrow 0}\left\|\int_{\varepsilon \leq|y| \leq 1} \vec{K}(y) d y-\vec{K}_{0}\right\|_{\mathbb{R} \rightarrow \ell^{2}}=0$.

Condition i) is clear because if $k_{0}(x)$ is the integer such that $2^{k_{0}(x)}<1 /|x|$ and $2^{k_{0}(x)+1} \geq 1 /|x|$, and we use again that $|\psi(x)| \leq B_{\psi, n} /(1+|x|)^{n+1} \leq B_{\psi, n} /|x|^{n+1}$, then

$$
\begin{aligned}
\|\vec{K}(x)\|_{\mathbb{R} \rightarrow \ell^{2}} & =\sup _{|a|=1}\left(\sum_{k \in \mathbb{Z}}\left(\psi_{2^{-k}}(x) a\right)^{2}\right)^{1 / 2}=\left(\sum_{k \in \mathbb{Z}}\left(2^{n k} \psi\left(2^{k} x\right)\right)^{2}\right)^{1 / 2} \\
& \leq\left(\sum_{k \leq k_{0}(x)}\left(2^{n k} \psi\left(2^{k} x\right)\right)^{2}+\sum_{k>k_{0}(x)}\left(2^{n k} \psi\left(2^{k} x\right)\right)^{2}\right)^{1 / 2} \\
& \leq\left(S_{\psi} \sum_{k \leq k_{0}(x)} 2^{2 n k}+B_{\psi, n} \sum_{k>k_{0}(x)}\left(2^{n k} \frac{1}{2^{(n+1) k}|x|^{n+1}}\right)^{2}\right)^{1 / 2} \\
& =\left(S_{\psi} \frac{2^{2 n k_{0}(x)}}{1-\frac{1}{2^{n}}}+B_{\psi, n} \frac{1}{|x|^{2(n+1)}} \sum_{k>k_{0}(x)} \frac{1}{2^{2 k}}\right)^{1 / 2} \\
& \leq\left(\frac{2^{n} S_{\psi}}{2^{n}-1} \frac{1}{|x|^{2 n}}+\frac{4 B_{\psi, n}}{3} \frac{1}{|x|^{2(n+1)}} \frac{1}{2^{2\left(k_{0}+1\right)}}\right)^{1 / 2} \\
& \leq\left(\frac{2^{n} S_{\psi}}{2^{n}-1} \frac{1}{|x|^{2 n}}+\frac{4 B_{\psi, n}}{3} \frac{1}{|x|^{2 n}}\right)^{1 / 2} \leq \tilde{S}_{\psi} \frac{1}{|x|^{n}}
\end{aligned}
$$

where

$$
S_{\psi}=\sup _{|y|<1}(\psi(y))^{2}, \quad \tilde{S}_{\psi, n}^{2}=\max \left\{\frac{2^{n} S_{\psi}}{2^{n}-1}, \frac{4 B_{\psi, n}}{3}\right\} .
$$


Condition iii) is also clear taking $\vec{K}_{0}=\int_{0 \leq|y| \leq 1} \vec{K}(y) d y$. Thus, let us focus on the second condition. We have that, given $|x| \geq 2|y|$, if

$$
g(t)=\psi\left(2^{k}(t(x-y)+(1-t) x)\right)=\psi\left(2^{k}(x-t y)\right)
$$

with $t \in[0,1]$, then

$$
\left|\psi_{2^{-k}}(x-y)-\psi_{2^{-k}}(x)\right|=2^{n k}|g(1)-\psi(0)|=2^{n k}\left|g^{\prime}\left(t_{0}\right)\right|,
$$

for some $t_{0} \in[0,1]$, and so using the chain rule, Cauchy-Schwarz inequality, and $|\nabla \psi(x)| \leq B_{\psi, n} /(1+|x|)^{n+1}$, we get

$$
\begin{aligned}
\left|\psi_{2^{-k}}(x-y)-\psi_{2^{-k}}(x)\right| & =2^{n k}\left|\left\langle\nabla \psi\left(2^{k}\left(x-t_{0} y\right)\right), 2^{k} y\right\rangle\right| \\
& =2^{(n+1) k}\left|\left\langle\nabla \psi\left(2^{k}\left(x-t_{0} y\right)\right), y\right\rangle\right| \\
& \leq 2^{(n+1) k}\left|\nabla \psi\left(2^{k}\left(x-t_{0} y\right)\right)\right||y| \\
& \leq 2^{(n+1) k} B_{\psi, n} \frac{1}{\left(1+2^{k}\left|x-t_{0} y\right|\right)^{n+1}}|y| \\
& \leq 2^{(n+1) k} B_{\psi, n} \frac{1}{\left(1+2^{k-1}|x|\right)^{n+1}}|y|,
\end{aligned}
$$

where the last inequality holds because $\left|x-t_{0} y\right| \geq|x|-|y| \geq|x|-|x| / 2=|x| / 2$. On the other hand, using again that $|x-y| \geq|x| / 2$, we also have

$$
\begin{aligned}
\left|\psi_{2^{-k}}(x-y)-\psi_{2^{-k}}(x)\right| & \leq 2^{n k}\left|\psi\left(2^{k}(x-y)\right)\right|+2^{n k}\left|\psi\left(2^{k} x\right)\right| \\
& \leq 2^{n k} B_{\psi, n} \frac{1}{\left(1+2^{k}|x-y|\right)^{n+1}}+2^{n k} B_{\psi, n} \frac{1}{\left(1+2^{k}|x|\right)^{n+1}} \\
& \leq 2^{n k} B_{\psi, n} \frac{1}{\left(1+2^{k-1}|x|\right)^{n+1}}+2^{n k} B_{\psi, n} \frac{1}{\left(1+2^{k}|x|\right)^{n+1}} \\
& \leq 2 B_{\psi, n} 2^{n k} \frac{1}{\left(1+2^{k-1}|x|\right)^{n+1}} .
\end{aligned}
$$

Therefore, for any $\gamma \in[0,1]$,

$$
\begin{aligned}
\left|\psi_{2^{-k}}(x-y)-\psi_{2^{-k}}(x)\right| & =\left|\psi_{2^{-k}}(x-y)-\psi_{2^{-k}}(x)\right|^{\gamma}\left|\psi_{2^{-k}}(x-y)-\psi_{2^{-k}}(x)\right|^{1-\gamma} \\
& \leq\left(\frac{2^{(n+1) k} B_{\psi, n}}{\left(1+2^{k-1}|x|\right)^{n+1}}|y|\right)^{\gamma}\left(\frac{2 B_{\psi, n} 2^{n k}}{\left(1+2^{k-1}|x|\right)^{n+1}}\right)^{1-\gamma} \\
& \leq 2^{n k} B_{\psi, n} \frac{1}{\left(1+2^{k-1}|x|\right)^{n+1}} 2^{k \gamma}|y|^{\gamma} 2^{1-\gamma}
\end{aligned}
$$

and so, if $k_{0}(x)$ is the integer such that $2^{k_{0}(x)}<2 /|x|$ and $2^{k_{0}(x)+1} \geq 2 /|x|$, we can write 


$$
\begin{aligned}
\|\vec{K}(x-y)-\vec{K}(x)\|_{\mathbb{R} \rightarrow \ell^{2}} & =\left(\sum_{k \in \mathbb{Z}}\left|\psi_{2^{-k}}(x-y)-\psi_{2^{-k}}(x)\right|^{2}\right)^{1 / 2} \\
& \leq \sum_{k \in \mathbb{Z}}\left|\psi_{2^{-k}}(x-y)-\psi_{2^{-k}}(x)\right| \\
& \leq \sum_{k \leq k_{0}(x)}\left|\psi_{2^{-k}}(x-y)-\psi_{2^{-k}}(x)\right| \\
& +\sum_{k>k_{0}(x)}\left|\psi_{2^{-k}}(x-y)-\psi_{2^{-k}}(x)\right| \\
& \leq|y| B_{\psi, n} \sum_{k \leq k_{0}(x)} \frac{2^{(n+1) k}}{\left(1+2^{k-1}|x|\right)^{n+1}} \\
& +|y|^{1 / 2} 2^{1 / 2} B_{\psi, n} \sum_{k>k_{0}(x)} \frac{2^{(n+1 / 2) k}}{\left(1+2^{k-1}|x|\right)^{n+1}},
\end{aligned}
$$

where we are using the last estimate with $\gamma=1$ in the first sum, and with $\gamma=1 / 2$ in the second one. From here, get in the end

$$
\begin{aligned}
\|\vec{K}(x-y)-\vec{K}(x)\|_{\mathbb{R} \rightarrow \ell^{2}} & \leq|y| B_{\psi, n} \sum_{k \leq k_{0}(x)} 2^{(n+1) k}+|y|^{1 / 2} 2^{1 / 2} B_{\psi, n} \sum_{k>k_{0}(x)} \frac{2^{(n+1 / 2) k}}{\left(2^{k-1}|x|\right)^{n+1}} \\
& \leq|y| B_{\psi, n} \frac{2^{(n+1) k_{0}(x)}}{1-\frac{1}{2^{n+1}}}+\frac{|y|^{1 / 2} 2^{n+3 / 2} B_{\psi, n}}{|x|^{n+1}} \sum_{k>k_{0}(x)} \frac{1}{2^{k / 2}} \\
& \leq|y| B_{\psi, n} \frac{2^{n+1}}{|x|^{n+1}\left(1-\frac{1}{2^{n+1}}\right)}+\frac{|y|^{1 / 2} 2^{n+3 / 2} B_{\psi, n}}{|x|^{n+1}} \frac{2^{-\left(k_{0}+1\right) / 2}}{1-\frac{1}{2^{1 / 2}}} \\
& \leq|y| B_{\psi, n} \frac{2^{n+1}}{|x|^{n+1}\left(1-\frac{1}{2^{n+1}}\right)}+\frac{|y|^{1 / 2} 2^{n+3 / 2} B_{\psi, n}}{|x|^{n+1}} \frac{|x|^{1 / 2}}{\sqrt{2}-1} \\
& \leq C_{n} B_{\psi, n}\left(\frac{|y|}{|x|^{n+1}}+\frac{|y|^{1 / 2}}{|x|^{n+1 / 2}}\right),
\end{aligned}
$$

taking

$$
C_{n}=\max \left\{\frac{2^{2(n+1)}}{2^{n+1}-1}, \frac{2^{n+3 / 2}}{\sqrt{2}-1}\right\},
$$

and thus finally, with this estimate, we get that

$$
\begin{aligned}
\int_{|x| \geq 2|y|}\|\vec{K}(x-y)-\vec{K}(x)\|_{\mathbb{R} \rightarrow \ell^{2}} d x & \leq C_{n} B_{\psi, n} \int_{|x| \geq 2|y|}\left(\frac{|y|}{|x|^{n+1}}+\frac{|y|^{1 / 2}}{|x|^{n+1 / 2}}\right) d x \\
& =\omega_{n} C_{n} B_{\psi, n} \int_{r \geq 2|y|}\left(\frac{|y|}{r^{2}}+\frac{|y|^{1 / 2}}{r^{3 / 2}}\right) d r \\
& =\omega_{n} C_{n} B_{\psi, n} \frac{1+2 \sqrt{2}}{2}
\end{aligned}
$$


so condition 2 of Theorem 1.10 follows. All in all, we can apply this theorem, and conclude that $\vec{T}$ is bounded from $L^{p}$ to $L^{p}\left(\mathbb{R}^{n}, \ell^{2}\right)$, for $1<p<\infty$, and bounded from $L^{1}$ to $L^{1, \infty}\left(\mathbb{R}^{n}, \ell^{2}\right)$, which means that $s_{\psi}$ is strong type $(p, p)$, for any $1<p<\infty$ and weak type $(1,1)$.

We focus now on the second part of the theorem. First, let $\Delta_{k}^{*}$ be the adjoint operator of $\Delta_{k}$; that is, $\Delta_{k}^{*}$ is such that

$$
\left\langle\Delta_{k} f, g\right\rangle=\left\langle f, \Delta_{k}^{*} g\right\rangle
$$

for any pair of functions $f, g$. Notice that, because $f, g$ are real valued functions, we have that

$$
\begin{aligned}
\left\langle\Delta_{k} f, g\right\rangle & =\int_{\mathbb{R}^{n}}\left(\Delta_{k} f\right)(x) g(x) d x=\int_{\mathbb{R}^{n}} g(x) \int_{\mathbb{R}^{n}} f(y) \psi_{2^{-k}}(x-y) d y d x \\
& =\int_{\mathbb{R}^{n}} f(y) \int_{\mathbb{R}^{n}} g(x) \psi_{2^{-k}}(x-y) d x d y,
\end{aligned}
$$

so we conclude that $\Delta_{k}^{*} g=g * \widetilde{\psi_{2^{-k}}}{ }^{1}$. Now, let $f \in L^{p}$, with $1<p<\infty$, and consider the series

$$
\Lambda=\sum_{k \in \mathbb{Z}} \Delta_{k}^{*} \Delta_{k} f
$$

We claim that $\Lambda$ converges in $\mathcal{S}^{\prime}$ to $f$. We might prove instead that $\widehat{\Lambda}$ converges in $\mathcal{S}^{\prime}$ to $\widehat{f}$, for the Fourier transform is a bijective continuous map in $\mathcal{S}^{\prime}$ whose inverse is also continuous (see section 1.4). We have that for any $g \in \mathcal{S}$,

$$
\begin{aligned}
\left(\sum_{|k| \leq N} \widehat{\Delta_{k}^{*} \Delta_{k}} f\right)(g) & =\left(\sum_{|k| \leq N} \widehat{\Delta_{k}^{*} \Delta_{k}} f\right)(g)=\left(\sum_{|k| \leq N} \widehat{f}\left|\widehat{\psi_{2^{-k}}}\right|\right)(g) \\
& =\int_{\mathbb{R}^{n}} \widehat{f}(x) g(x) \sum_{|k| \leq N}\left|\widehat{\psi_{2^{-k}}}(x)\right| d x,
\end{aligned}
$$

but, by hypothesis,

$$
\int_{\mathbb{R}^{n}} \widehat{f}(x) g(x) \sum_{|k| \leq N}\left|\widehat{\psi_{2^{-k}}}(x)\right| d x \stackrel{N \rightarrow \infty}{\longrightarrow} \int_{\mathbb{R}^{n}} \widehat{f}(x) g(x) d x=\widehat{f}(g),
$$

in virtue of the dominated convergence theorem. Thus, we have seen that $\widehat{\Lambda}$ converges to $\widehat{f}$ pointwise, and so by Banach-Steinhaus theorem [1, Theorem 3.15] we have that $\widehat{\Lambda}$ converges to $\widehat{f}$ in $\mathcal{S}^{\prime}$. With this, we finally have that

\footnotetext{
${ }^{1}$ Notice that if $\psi$ was symmetric, then $\Delta_{k}$ would be self-adjoint. Also, for complex valued functions, $\Delta_{k}^{*}$ would be simply given by $\widehat{\Delta_{k}^{*} f}=\widehat{f} \widehat{\widehat{\psi_{2^{-k}}}}$.
} 


$$
\begin{aligned}
& \|f\|_{p}=\sup _{\substack{g \in L^{p^{\prime}} \\
\|g\|_{p^{\prime}}=1}}\left|\int_{\mathbb{R}^{n}} f(x) g(x) d x\right|=\sup _{\substack{g \in \mathcal{S} \\
\|g\|_{p^{\prime}}=1}}\left|\int_{\mathbb{R}^{n}} f(x) g(x) d x\right| \\
& =\sup _{\substack{g \in \mathcal{S} \\
\|g\|_{p^{\prime}}=1}}|f(g)|=\sup _{\substack{g \in \mathcal{S} \\
\|g\|_{p^{\prime}}=1}}|\Lambda(g)|=\sup _{\substack{g \in \mathcal{S} \\
\|g\|_{p^{\prime}}=1}}\left|\left(\sum_{k \in \mathbb{Z}} \Delta_{k}^{*} \Delta_{k} f\right)(g)\right| \\
& =\sup _{\substack{g \in \mathcal{S} \\
\|g\|_{p^{\prime}}=1}}\left|\sum_{k \in \mathbb{Z}}\left(\Delta_{k}^{*} \Delta_{k} f\right)(g)\right|=\sup _{\substack{g \in \mathcal{S} \\
\|g\|_{p^{\prime}}=1}}\left|\sum_{k \in \mathbb{Z}}\left\langle\Delta_{k}^{*} \Delta_{k} f, g\right\rangle\right| \\
& =\sup _{\substack{g \in \mathcal{S} \\
\|g\|_{p^{\prime}}=1}}\left|\sum_{k \in \mathbb{Z}}\left\langle\Delta_{k} f, \Delta_{k} g\right\rangle\right|=\sup _{\substack{g \in \mathcal{S} \\
\|g\|_{p^{\prime}}=1}}\left|\int_{\mathbb{R}^{n}} \sum_{k \in \mathbb{Z}}\left(\Delta_{k} f\right)(x)\left(\Delta_{k} g\right)(x) d x\right| \\
& \leq \sup _{\substack{g \in \mathcal{S} \\
\|g\|_{p^{\prime}}=1}} \int_{\mathbb{R}^{n}}\left|\sum_{k \in \mathbb{Z}}\left(\Delta_{k} f\right)(x)\left(\Delta_{k} g\right)(x)\right| d x \\
& \leq \sup _{\substack{g \in \mathcal{S} \\
\|g\|_{p^{\prime}}=1}} \int_{\mathbb{R}^{n}}\left(\sum_{k \in \mathbb{Z}}\left|\left(\Delta_{k} f\right)(x)\right|^{2}\right)^{1 / 2}\left(\sum_{k \in \mathbb{Z}}\left|\left(\Delta_{k} g\right)(x)\right|^{2}\right)^{1 / 2} d x \\
& \leq \sup _{\substack{g \in \mathcal{S} \\
\|g\|_{p^{\prime}}=1}}\left\|\left(\sum_{k \in \mathbb{Z}}\left|\Delta_{k} f\right|^{2}\right)^{1 / 2}\right\|\left\|\left(\sum_{k \in \mathbb{Z}}\left|\Delta_{k} g\right|^{2}\right)^{1 / 2}\right\|_{p^{\prime}} \\
& \lesssim \sup _{\substack{g \in \mathcal{S} \\
\|g\|_{p^{\prime}}=1}}\left\|\left(\sum_{k \in \mathbb{Z}}\left|\Delta_{k} f\right|^{2}\right)^{1 / 2}\right\|_{p}\|g\|_{p^{\prime}}=\left\|\left(\sum_{k \in \mathbb{Z}}\left|\Delta_{k} f\right|^{2}\right)^{1 / 2}\right\|_{p},
\end{aligned}
$$

where the inequalities used are, in order, the triangular inequality, Cauchy-Schwarz in $\ell^{2}$, Hölder's, and the fact that the square function $s_{\psi}$ is strong type $\left(p^{\prime}, p^{\prime}\right)$, in virtue of the first part of this theorem. Thus, part (ii) of the theorem is seen, and the proof is finally complete.

\subsection{Orthogonal decomposition in dyadic sets}

In our initial discussion, the functions $\psi_{2^{-k}}$ were characteristic functions of annuli. The theorem we have just proved, though, requires $\psi$ to be a very nice function. We want to see now if we can adapt the result so that it works as well for non-smooth functions.

Let us begin with the case $n=1$. Given $k \in \mathbb{Z}$, we denote by $\chi_{k}$ the characteristic function of the dyadic set $\left(-2^{k+1},-2^{k}\right] \cup\left[2^{k}, 2^{k+1}\right)$, and we define the Littlewood- 
Paley operator associated to $\chi_{k}$ as the operator $\Delta_{k}^{\#}$ given by

$$
\widehat{\Delta_{k}^{\#} f}=\widehat{f} \chi_{k} .
$$

Notice, by Example 2.20, that we can write

$$
\Delta_{k}^{\#} f=\frac{i}{2}\left[\left(e_{2^{k+1}} H e_{-2^{k+1}}-e_{2^{k}} H e_{-2^{k}}\right) f+\left(e_{-2^{k}} H e_{2^{k}}-e_{-2^{k+1}} H e_{2^{k+1}}\right) f\right] .
$$

With this, we have the following result.

Theorem 3.5. Given $1<p<\infty$, it holds that

$$
\|f\|_{p} \approx\left\|\left(\sum_{k \in \mathbb{Z}}\left|\Delta_{k}^{\#} f\right|^{2}\right)^{1 / 2}\right\|_{p}, \quad \forall f \in L^{p}(\mathbb{R}) .
$$

Proof. Let $\psi \in \mathcal{S}(\mathbb{R})$ be such that its Fourier transform is supported in $2^{-1} \leq|\xi| \leq 2^{2}$, with $\widehat{\psi}(\xi)=1$ for all $1 \leq|\xi| \leq 2$. Then, each function $\widehat{\psi_{2^{-k}}}$ will be, by definition of $\psi_{2^{-k}}$, supported in $2^{k-1} \leq|\xi| \leq 2^{k+2}$, and will equal 1 in $2^{k} \leq|\xi| \leq 2^{k+1}$. Now, let $\Delta_{k}$ be the Littlewood-Paley operator associated to $\psi_{2^{-k}}$. With this, it holds that

$$
\Delta_{k} \Delta_{k}^{\#}=\Delta_{k}^{\#} \Delta_{k}=\Delta_{k}^{\#} .
$$

Indeed, the first equality is trivial taking Fourier transform. The second one is also clear, again taking Fourier transform, and because $\widehat{\psi_{2^{-k}}}$ equals 1 on the support of $\chi_{k}$, so that $\widehat{f} \widehat{\psi_{2^{-k}}} \chi_{k}=\widehat{f} \chi_{k}$, for any function $f$. We can then write

$$
\begin{aligned}
\left\|\left(\sum_{k \in \mathbb{Z}}\left|\Delta_{k}^{\#} f\right|^{2}\right)^{1 / 2}\right\|_{p} & \left\|\left(\sum_{k \in \mathbb{Z}}\left|\Delta_{k}^{\#} \Delta_{k} f\right|^{2}\right)^{1 / 2}\right\| \|_{p} \\
\leq & \left\|\left(\sum_{k \in \mathbb{Z}}\left|\frac{i}{2}\left(e_{2^{k+1}} H e_{-2^{k+1}}-e_{2^{k}} H e_{-2^{k}}\right) \Delta_{k} f\right|^{2}\right)^{1 / 2}\right\|_{p} \\
& +\left\|\left(\sum_{k \in \mathbb{Z}}\left|\frac{i}{2}\left(e_{-2^{k}} H e_{2^{k}}-e_{-2^{k+1}} H e_{2^{k+1}}\right) \Delta_{k} f\right|^{2}\right)^{1 / 2}\right\| \\
\leq & \frac{1}{2}\left\|\left(\sum_{k \in \mathbb{Z}}\left|e_{2^{k+1}} H e_{-2^{k+1}} \Delta_{k} f\right|^{2}\right)^{1 / 2}\right\|_{p} \\
& +\frac{1}{2}\left\|\left(\sum_{k \in \mathbb{Z}}\left|e_{2^{k}} H e_{-2^{k}} \Delta_{k} f\right|^{2}\right)^{1 / 2}\right\|_{p} \\
& +\frac{1}{2}\left\|\left(\sum_{k \in \mathbb{Z}}\left|e_{-2^{k}} H e_{2^{k}} \Delta_{k} f\right|^{2}\right)^{1 / 2}\right\|_{p} \\
& +\frac{1}{2} \|\left(\sum_{k \in \mathbb{Z}}\left|e_{-2^{k+1}} H e_{2^{k+1}} \Delta_{k} f\right|^{2} \|_{p}\right.
\end{aligned}
$$


Notice that, for the first term (and similarly for the other three), we can write $g_{k}=e_{-2^{k+1}} \Delta_{k} f$, and it holds

$$
\begin{aligned}
\left|g_{k}\right| & =\left|\Delta_{k} f\right| \\
\left|e_{2^{k+1}} H g_{k}\right| & =\left|H g_{k}\right|,
\end{aligned}
$$

because the operators $e_{-2^{k+1}}$ and $e_{2^{k+1}}$ only introduce factors of modulus 1 . Thus, because the Hilbert transform is bounded from $L^{p}$ to $L^{p}$, we can apply Theorem 1.9 and get

$$
\begin{aligned}
\left\|\left(\sum_{k \in \mathbb{Z}}\left|e_{2^{k+1}} H e_{-2^{k+1}} \Delta_{k} f\right|^{2}\right)^{1 / 2}\right\|_{p} & =\left\|\left(\sum_{k \in \mathbb{Z}}\left|H g_{k}\right|^{2}\right)^{1 / 2}\right\|_{p} \\
& \lesssim\left\|\left(\sum_{k \in \mathbb{Z}}\left|g_{k}\right|^{2}\right)^{1 / 2}\right\|_{p} \\
& =\left\|\left(\sum_{k \in \mathbb{Z}}\left|\Delta_{k} f\right|^{2}\right)^{1 / 2}\right\|_{p}
\end{aligned}
$$

and by Theorem 3.4, we finally conclude

$$
\left\|\left(\sum_{k \in \mathbb{Z}}\left|\Delta_{k}^{\#} f\right|^{2}\right)^{1 / 2}\right\|_{p} \lesssim\left\|\left(\sum_{k \in \mathbb{Z}}\left|\Delta_{k} f\right|^{2}\right)^{1 / 2}\right\|_{p} \lesssim\|f\|_{p} .
$$

The reverse inequality is proven using the exact same argument as the one developed when proving part (ii) of Theorem 3.4.

Let us now try to give a version of this previous result for $n \geq 2$. We pick a multiindex tuple $\vec{k}=\left(k_{1}, \ldots, k_{n}\right) \in \mathbb{Z}^{n}$, and we define $\chi_{\vec{k}}$ to be the characteristic function of the dyadic set

$$
\begin{aligned}
I_{\vec{k}} & =I_{k_{1}} \times \cdots \times I_{k_{n}} \\
& =\left\{\left(x_{1}, \ldots, x_{n}\right) \in \mathbb{R}^{n}: x_{i} \in\left(-2^{k_{i}+1},-2^{k_{i}}\right] \cup\left[2^{k_{i}}, 2^{k_{i}+1}\right) \forall i=1, \ldots, n\right\},
\end{aligned}
$$

where $I_{k_{i}}=\left(-2^{k_{i}+1},-2^{k_{i}}\right] \cup\left[2^{k_{i}}, 2^{k_{i}+1}\right)$. Each set $I_{\vec{k}}$ is a union of $2^{n}$ rectangles (see figures 3.1 and 3.2). Moreover, $I_{\vec{k}}$ and $I_{\overrightarrow{k^{\prime}}}$ are disjoint whenever $\vec{k} \neq \overrightarrow{k^{\prime}}$. Thus, $\left\{I_{\vec{k}}\right\}_{\vec{k} \in \mathbb{Z}^{n}}$ is a partition of $\mathbb{R}^{n} \backslash\{0\}$, and so

$$
\sum_{\vec{k} \in \mathbb{Z}^{n}} \chi_{\vec{k}}(x)=1, \quad \forall x \in \mathbb{R}^{n} \backslash\{0\} .
$$

Finally, define the Littlewood-Paley operator associated to $\chi_{\vec{k}}$ as the operator $\Delta_{\vec{k}}^{\#}$ given by

$$
\widehat{\Delta_{\vec{k}}^{\#} f}=\widehat{f} \chi_{\vec{k}} .
$$




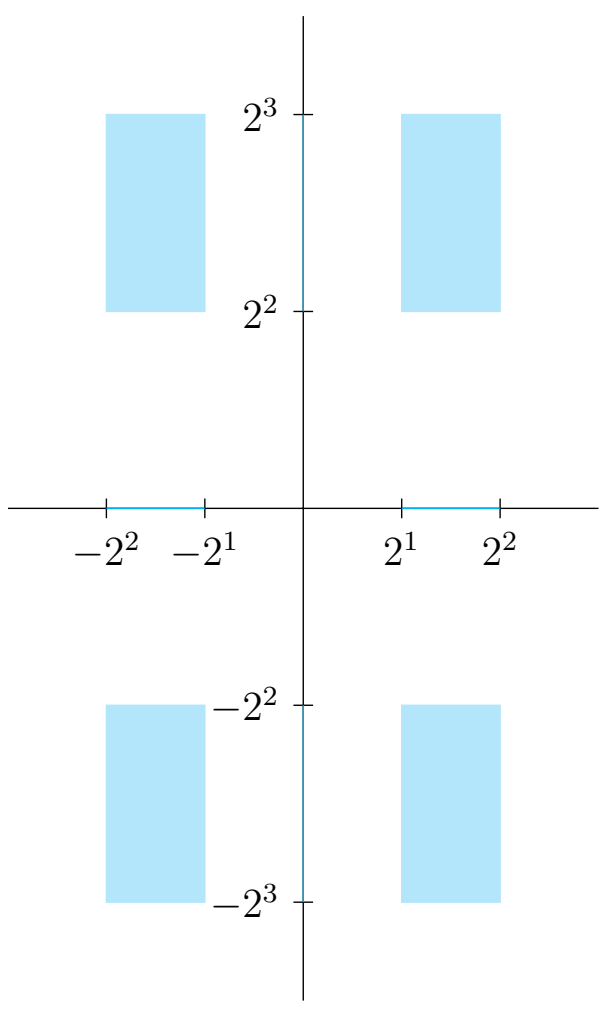

Figure 3.1: $I_{\vec{k}}$ for $\vec{k}=(1,2)$

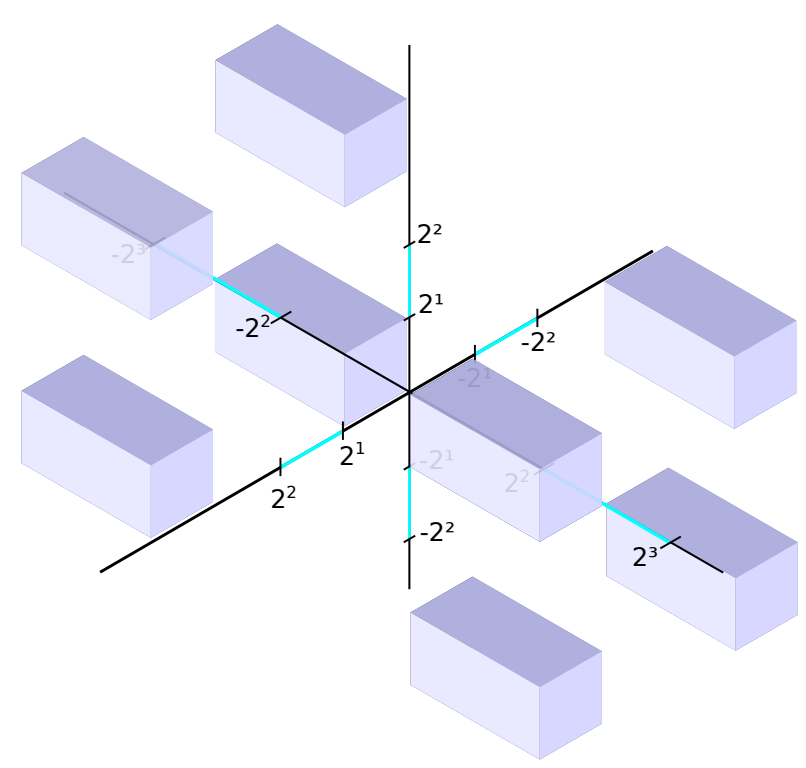

Figure 3.2: $I_{\vec{k}}$ for $\vec{k}=(1,2,1)$

In order to give an $n$-dimensional version of Theorem 3.5, we shall try to redo the proof of the theorem, and so we might need to relate the operators $\Delta_{\vec{k}}^{\#}$ with Littlewood-Paley operators associated to functions in $\mathcal{S}$. But notice as well that

$$
\chi_{\vec{k}}(x)=\chi_{k_{1}}\left(x_{1}\right) \cdots \chi_{k_{n}}\left(x_{n}\right), \quad \forall x \in \mathbb{R}^{n}
$$

that is, the multiplier associated to $\Delta_{\vec{k}}^{\#}$ is actually a product of $n$ multipliers, each one associated to one distinct variable. That is not the case, in general, for the Littlewood-Paley multipliers of Definition 3.2. Thus, we introduce another type of Littlewood-Paley operators, denoted as $\Delta_{\vec{k}}$ (notice the multiindex $\vec{k}$ ), which are given by

$$
\widehat{\Delta_{\vec{k}}} f(\xi)=\widehat{f}(\xi) \widehat{\psi_{2^{-k_{1}}}}\left(\xi_{1}\right) \cdots \widehat{\psi_{2^{-k_{n}}}}\left(\xi_{n}\right)=\widehat{f}(\xi) \widehat{\psi}\left(2^{-k_{1}} \xi_{1}\right) \cdots \widehat{\psi}\left(2^{-k_{n}} \xi_{n}\right),
$$

where $\psi \in \mathcal{S}(\mathbb{R})$. With this, the operators $\Delta_{\vec{k}}$ and $\Delta_{\vec{k}}^{\#}$ can be related in a similar fashion as in the proof of the previous theorem, although we now encounter another difficulty, namely, that we cannot use Theorem 3.4 directly. Fortunately, this problem can be overcome.

Lemma 3.6. Let $\Delta_{\vec{k}}$ be the $\vec{k}$-th multiindex Littlewood-Paley operator associated to a Schwarz function $\psi \in \mathcal{S}(\mathbb{R})$, for each $\vec{k} \in \mathbb{Z}^{n}$, where $\psi$ is such that

$$
\int_{\mathbb{R}} \psi(x) d x=0 .
$$


Then, the square function associated to $\psi$,

$$
s_{\psi}(f)=\left(\sum_{\vec{k} \in \mathbb{Z}^{n}}\left|\Delta_{\vec{k}}(f)\right|^{2}\right)^{1 / 2},
$$

is strong type $(p, p)$ for any $1<p<\infty$.

Proof. For $i=1, \ldots, n$, define $\psi^{i} \in \mathcal{S}\left(\mathbb{R}^{n}\right)$ as the function such that

$$
\widehat{\psi^{i}}(\xi)=\widehat{\psi}\left(\xi_{i}\right), \quad \forall \xi \in \mathbb{R}^{n}
$$

Notice that

$$
\widehat{\psi_{2^{-k_{i}}}^{i}}(\xi)=\widehat{\psi^{i}}\left(2^{-k_{i}} \xi\right)=\widehat{\psi}\left(2^{-k_{i}} \xi_{i}\right)
$$

which means that, if

$$
\widehat{\Delta_{\vec{k}} f}(\xi)=\widehat{f}(\xi) \widehat{\psi}\left(2^{-k_{1}} \xi_{1}\right) \cdots \widehat{\psi}\left(2^{-k_{n}} \xi_{n}\right)=\widehat{f}(\xi) \widehat{\psi_{2^{-k_{1}}}^{1}}(\xi) \cdots \widehat{\psi_{2^{-k_{n}}}^{n}}(\xi),
$$

then

$$
\Delta_{\vec{k}}(f)=\Delta_{k_{1}}^{1} \cdots \Delta_{k_{n}}^{n}(f),
$$

where $\Delta_{k_{i}}^{i}$ is the $k_{i}$-th Littlewood-Paley operator associated to $\psi^{i}$.

We will prove the lemma by induction on $n$. The case $n=1$ is already covered by Theorem 3.4, so let us see the case $n=2$. We want to verify that for $1<p<\infty$,

$$
\left\|\left(\sum_{\vec{k} \in \mathbb{Z}^{2}}\left|\Delta_{\vec{k}}(f)\right|^{2}\right)^{1 / 2}\right\|_{p}=\left\|\left(\sum_{k_{2} \in \mathbb{Z}} \sum_{k_{1} \in \mathbb{Z}}\left|\Delta_{k_{1}}^{1} \Delta_{k_{2}}^{2}(f)\right|^{2}\right)^{1 / 2}\right\|_{p} \lesssim\|f\|_{p}
$$

but it follows, with some technicalities, from Theorem 1.10 (see [5, Proposition 6.1.4]) that

$$
\left\|\left(\sum_{k_{2} \in \mathbb{Z}} \sum_{k_{1} \in \mathbb{Z}}\left|\Delta_{k_{1}}^{1} \Delta_{k_{2}}^{2}(f)\right|^{2}\right)^{1 / 2}\right\|_{p}\|\|\left(\sum_{k_{2} \in \mathbb{Z}}\left|\Delta_{k_{2}}^{2}(f)\right|^{2}\right)^{1 / 2} \|_{p},
$$

and so applying now the usual estimate given by Theorem 3.4, we get the result. The induction step is simply done using again [5, Proposition 6.1.4] and a suitable enumeration of $\mathbb{Z}^{n-1}$, since the sum is of positive terms.

Now we are ready to prove Theorem 3.5 for $n \geq 2$.

Theorem 3.7. Given $1<p<\infty$, it holds that

$$
\|f\|_{p} \approx\left\|\left(\sum_{\vec{k} \in \mathbb{Z}^{n}}\left|\Delta_{\vec{k}}^{\#} f\right|^{2}\right)^{1 / 2}\right\|_{p}, \quad \forall f \in L^{p}\left(\mathbb{R}^{n}\right) .
$$


Proof. For the sake of simplicity, we will do the proof for $n=2$. The general case follows exactly in the same way. Let $\psi \in \mathcal{S}(\mathbb{R})$ be such that its Fourier transform is supported in $2^{-1} \leq|\xi| \leq 2^{2}$, with $\widehat{\psi}(\xi)=1$ for all $1 \leq|\xi| \leq 2$. Then, each function $\widehat{\psi_{2^{-k}}}$ will be, by definition of $\psi_{2^{-k}}$, supported in $2^{k-1} \leq|\xi| \leq 2^{k+2}$, and will equal 1 in $2^{k} \leq|\xi| \leq 2^{k+1}$. In particular, the function

$$
\widehat{\psi_{2^{-k_{1}}}^{1}}(\xi) \widehat{\psi_{2^{-k_{2}}}^{2}}(\xi)=\widehat{\psi^{1}}\left(2^{-k_{1}} \xi\right) \widehat{\psi^{2}}\left(2^{-k_{2}} \xi\right)=\widehat{\psi}\left(2^{-k_{1}} \xi_{1}\right) \widehat{\psi}\left(2^{-k_{2}} \xi_{2}\right)=\widehat{\psi_{2^{-k_{1}}}}\left(\xi_{1}\right) \widehat{\psi_{2^{-k_{2}}}}\left(\xi_{2}\right)
$$

is clearly supported in the set

$$
S=\left\{\xi \in \mathbb{R}^{2}: 2^{k_{1}-1} \leq\left|\xi_{1}\right| \leq 2^{k_{1}+2}, 2^{k_{2}-1} \leq\left|\xi_{2}\right| \leq 2^{k_{2}+2}\right\},
$$

and will be equal to 1 in the set

$$
I_{\vec{k}}=I_{k_{1}} \times I_{k_{2}}
$$

with $\vec{k}=\left(k_{1}, k_{2}\right)$. Thus, if $\Delta_{\vec{k}}$ is the multiindex Littlewood-Paley operator associated to $\psi$, and $\Delta_{\vec{k}}^{\#}$ is the Littlewood-Paley operator associated to $\chi_{\vec{k}}$, it holds

$$
\Delta_{\vec{k}}^{\#} \Delta_{\vec{k}}=\Delta_{\vec{k}}^{\#}
$$

Now, recall that $\Delta_{\vec{k}}^{\#}=\Delta_{k_{1}}^{1, \#} \Delta_{k_{2}}^{2, \#}$, where $\Delta_{k_{j}}^{j, \#}$ is the Littlewood-Paley operator associated to $\chi_{k_{j}}\left(\xi_{j}\right)$, so

$$
\begin{aligned}
\left\|\left(\sum_{\vec{k} \in \mathbb{Z}^{2}}\left|\Delta_{\vec{k}}^{\#} f\right|^{2}\right)^{1 / 2}\right\|_{p} & =\left\|\left(\sum_{\vec{k} \in \mathbb{Z}^{2}}\left|\Delta_{\vec{k}}^{\#} \Delta_{\vec{k}} f\right|^{2}\right)^{1 / 2}\right\|_{p} \\
& =\left\|\left(\sum_{k_{1} \in \mathbb{Z}} \sum_{k_{2} \in \mathbb{Z}}\left|\Delta_{k_{1}}^{1, \#} \Delta_{k_{2}}^{2, \#} \Delta_{\vec{k}} f\right|^{2}\right)^{1 / 2}\right\|_{p} .
\end{aligned}
$$

As in the proof of Theorem 3.5, we can write

$$
\begin{aligned}
\Delta_{k_{j}}^{j, \#} & =\frac{i}{2}\left[e_{2^{k_{j}+1}} H^{j} e_{-2^{k_{j}+1}}-e_{2^{k_{j}}} H^{j} e_{-2^{k_{j}}}+e_{-2^{k_{j}}} H^{j} e_{2^{k_{j}}}-e_{-2^{k_{j}+1}} H^{j} e_{2^{k_{j}+1}}\right] \\
& :=\frac{i}{2}\left[T_{2^{k_{j}+1}}-T_{2^{k_{j}}}+T_{-2^{k_{j}}}-T_{-2^{k_{j}+1}}\right]
\end{aligned}
$$

where $H^{j}$ is the Hilbert transform acting on the $j$-th variable; that is, for $j=1$,

$$
H^{1} f\left(x_{1}, x_{2}\right)=\text { p.v. } \frac{1}{\pi} \int_{\mathbb{R}} \frac{f\left(y, x_{2}\right)}{x_{1}-y} d y,
$$

and similarly for $j=2$. Notice that $H^{j}$ bounded for $1<p<\infty$, for the Hilbert transform is bounded. With this, write 


$$
\begin{aligned}
\left\|\left(\sum_{k_{1} \in \mathbb{Z}} \sum_{k_{2} \in \mathbb{Z}}\left|\Delta_{k_{1}}^{1, \#} \Delta_{k_{2}}^{2, \#} \Delta_{\vec{k}} f\right|^{2}\right)^{1 / 2}\right\| & \left\|\left(\sum_{k_{1} \in \mathbb{Z}} \sum_{k_{2} \in \mathbb{Z}}\left|T_{2^{k_{j}+1}} \Delta_{k_{2}}^{2, \#} \Delta_{\vec{k}} f\right|^{2}\right)^{1 / 2}\right\|_{p} \\
+ & \left\|\left(\sum_{k_{1} \in \mathbb{Z}} \sum_{k_{2} \in \mathbb{Z}}\left|T_{2^{k_{j}}} \Delta_{k_{2}}^{2, \#} \Delta_{\vec{k}} f\right|^{2}\right)^{1 / 2}\right\|_{p} \\
& +\left\|\left(\sum_{k_{1} \in \mathbb{Z}} \sum_{k_{2} \in \mathbb{Z}}\left|T_{-2^{k_{j}}} \Delta_{k_{2}}^{2, \#} \Delta_{\vec{k}} f\right|^{2}\right)^{1 / 2}\right\|_{p} \\
& +\left\|\left(\sum_{k_{1} \in \mathbb{Z}} \sum_{k_{2} \in \mathbb{Z}}\left|T_{-2^{k_{j}+1}} \Delta_{k_{2}}^{2, \#} \Delta_{\vec{k}} f\right|^{2}\right)^{1 / 2}\right\|_{p}
\end{aligned}
$$

and for the first term (and similarly for the others), we can choose a suitable enumeration of $\mathbb{Z}^{2}$ and apply Theorem 1.9 so that

$$
\begin{aligned}
\left\|\left(\sum_{k_{1} \in \mathbb{Z}} \sum_{k_{2} \in \mathbb{Z}}\left|T_{2^{k_{j}+1}} \Delta_{k_{2}}^{2, \#} \Delta_{\vec{k}} f\right|^{2}\right)^{1 / 2}\right\|_{p} & =\left\|\left(\sum_{k_{1} \in \mathbb{Z}} \sum_{k_{2} \in \mathbb{Z}}\left|H^{1} e_{-2^{k_{j}+1}} \Delta_{k_{2}}^{2, \#} \Delta_{\vec{k}} f\right|^{2}\right)^{1 / 2}\right\|_{p} \\
& \lesssim\left\|\left(\sum_{k_{1} \in \mathbb{Z}} \sum_{k_{2} \in \mathbb{Z}}\left|e_{-2^{k_{j}+1}} \Delta_{k_{2}}^{2, \#} \Delta_{\vec{k}} f\right|^{2}\right)^{1 / 2}\right\|_{p} \\
& \lesssim\left\|\left(\sum_{k_{1} \in \mathbb{Z}} \sum_{k_{2} \in \mathbb{Z}}\left|\Delta_{k_{2}}^{2, \#} \Delta_{\vec{k}} f\right|^{2}\right)^{1 / 2}\right\|_{p}
\end{aligned}
$$

Repeating the same technique with $\Delta_{k_{2}}^{2, \#}$ and using Lemma 3.6 finally leads to

$$
\left\|\left(\sum_{k_{1} \in \mathbb{Z}} \sum_{k_{2} \in \mathbb{Z}}\left|\Delta_{k_{1}}^{1, \#} \Delta_{k_{2}}^{2, \#} \Delta_{\vec{k}} f\right|^{2}\right)^{1 / 2}\right\|_{p}\left\|\left(\sum_{k_{1} \in \mathbb{Z}} \sum_{k_{2} \in \mathbb{Z}}\left|\Delta_{\vec{k}} f\right|^{2}\right)^{1 / 2}\right\|_{p} \lesssim\|f\|_{p},
$$

and so one of the desired inequalities is proven. The reverse inequality, one more time, is proven as in the second part of Theorem 3.4. 


\section{Chapter 4}

\section{Multipliers theorems}

After reviewing Littlewood-Paley theory, we are now ready to give Marcinkiewicz's theorem and Mihlin-Hörmander's theorem. As we have said before, these results are sufficient conditions for determining when a certain $L^{\infty}$ function is actually an $M_{p}^{p}$ multiplier. On the one hand, these theorems represent a little improvement of some cases of multipliers that are trivially in $M_{p}^{p}$ (for instance, if $m \in \mathcal{S}$ ). The bad news is that they do not particularly help on the characterization of the $M_{p}^{p}$ spaces, because the conclusion, in both cases, is that $m \in M_{p}^{p}$ for all $1<p<\infty$. Also, they still assume the function $m$ to have certain regularity, like being piecewise $C^{1}$, and having some controlled growth of the derivatives. Nevertheless, we will see that these results are useful, for instance, when proving if a certain function is a multiplier, regarding that this function satisfy some homogeneity property.

\subsection{Marcinkiewicz's theorem}

We will start proving this theorem for $n=1$. After that, we will jump to the case $n \geq 2$.

Theorem 4.1 (Marcinkiewicz's theorem in $\mathbb{R}$ ). Let $m: \mathbb{R} \rightarrow \mathbb{R}$ be a bounded function that is $C^{1}$ in every dyadic set $O_{k}=\left(-2^{k+1},-2^{k}\right) \cup\left(2^{k}, 2^{k+1}\right)$, with $k \in \mathbb{Z}$, and such that

$$
\sup _{k \in \mathbb{Z}} \int_{O_{k}}\left|m^{\prime}(\xi)\right| d \xi:=A<\infty .
$$

Then, $m \in M_{p}^{p}$ for all $1<p<\infty$.

Proof. Without loss of generality, $m(\xi)=0$ for $\xi<0$. Indeed, if that was not the case, then write $m=m^{+}+m^{-}:=m \chi_{[0,+\infty)}+m \chi_{(-\infty, 0)}$, and because $m^{+}$and $\widetilde{m^{-}}$ satisfy $m(\xi)=0$ for $\xi<0$, they will be $M_{p}^{p}$ multipliers, and so will be $m$. Also, we may assume that $m$ is right continuous in $-2^{k}$ and left continuous in $2^{k}$ in each $O_{k}$. Indeed, this is so because

$$
\sup _{k \in \mathbb{Z}} \int_{O_{k}}\left|m^{\prime}(\xi)\right| d \xi<\infty
$$


implies that $m$ has bounded variation in any closed interval $[-M, M]$, for $M>0$, and so $m$ can be expressed as the difference of two increasing functions (see [10, Section 6.3]), meaning that $m$ can only have removable or jump discontinuities. Thus, right and left continuity in $-2^{k}$ and $2^{k}$, respectively, follows from a change of definition in these points.

Since $m^{\prime}$ is integrable over each interval $\left[2^{k}, 2^{k+1}\right)$, it follows from the fundamental theorem of calculus that

$$
m(\xi)=m\left(2^{k}\right)+\int_{2^{k}}^{\xi} m^{\prime}(t) d t
$$

for any $\xi \in\left[2^{k}, 2^{k+1}\right)$. In particular, for any $f \in \mathcal{S}$,

$$
\begin{aligned}
\widehat{f}(\xi) m(\xi) \chi_{k}(\xi) & =m\left(2^{k}\right) \widehat{f}(\xi) \chi_{k}(\xi)+\int_{2^{k}}^{\xi} \widehat{f}(\xi) \chi_{k}(\xi) m^{\prime}(t) d t \\
\widehat{f}(\xi) m(\xi) \chi_{k}(\xi) & =m\left(2^{k}\right) \widehat{f}(\xi) \chi_{k}(\xi)+\int_{2^{k}}^{2^{k+1}} \widehat{f}(\xi) \chi_{k}(\xi) \chi_{[t, \infty)}(\xi) m^{\prime}(t) d t \\
\left(\widehat{f} m \chi_{k}\right)^{\vee} & =m\left(2^{k}\right) \Delta_{k}^{\#} f+\int_{2^{k}}^{2^{k+1}} \Delta_{[t, \infty)} \Delta_{k}^{\#} f m^{\prime}(t) d t \\
\left|\left(\widehat{f} m \chi_{k}\right)^{\vee}\right| & \leq\|m\|_{\infty}\left|\Delta_{k}^{\#} f\right|+\int_{2^{k}}^{2^{k+1}}\left|\Delta_{[t, \infty)} \Delta_{k}^{\#} f\right|\left|m^{\prime}(t)\right| d t \\
\left|\left(\widehat{f} m \chi_{k}\right)^{\vee}\right| & \leq\|m\|_{\infty}\left|\Delta_{k}^{\#} f\right|+\int_{2^{k}}^{2^{k+1}}\left|\Delta_{[t, \infty)} \Delta_{\left\lfloor\log _{2} t\right]}^{\#} f\right|\left|m^{\prime}(t)\right| d t
\end{aligned}
$$

where $\lfloor a\rfloor$ denotes the integer part of any real number $a$. Using Cauchy-Schwarz inequality with $g_{1}=\left|\Delta_{[t, \infty)} \Delta_{\left\lfloor\log _{2} t\right\rfloor}^{\#} f\right|\left|m^{\prime}(t)\right|^{1 / 2}$ and $g_{2}=\left|m^{\prime}(t)\right|^{1 / 2}$, we can bound the integral on the right hand side of the last expression as

$$
\begin{aligned}
\int_{2^{k}}^{2^{k+1}}\left|\Delta_{[t, \infty)} \Delta_{\left\lfloor\log _{2} t\right\rfloor}^{\#} f\right|\left|m^{\prime}(t)\right| d t & \leq \int_{2^{k}}^{2^{k+1}}\left|m^{\prime}(t)\right| d t \int_{2^{k}}^{2^{k+1}}\left|\Delta_{[t, \infty)} \Delta_{\left\lfloor\log _{2} t\right\rfloor}^{\#} f\right|^{2}\left|m^{\prime}(t)\right| d t \\
& \leq A \int_{2^{k}}^{2^{k+1}}\left|\Delta_{[t, \infty)} \Delta_{\left\lfloor\log _{2} t\right\rfloor}^{\#} f\right|^{2}\left|m^{\prime}(t)\right| d t .
\end{aligned}
$$

This yields

$$
\begin{aligned}
\left(\sum_{k \in \mathbb{Z}}\left|\left(\widehat{f} m \chi_{k}\right)^{\vee}\right|^{2}\right)^{1 / 2} \leq & \|m\|_{\infty}\left(\sum_{k \in \mathbb{Z}}\left|\Delta_{k}^{\#} f\right|^{2}\right)^{1 / 2} \\
& +A^{1 / 2}\left(\int_{0}^{\infty}\left|\Delta_{[t, \infty)} \Delta_{\left\lfloor\log _{2} t\right\rfloor}^{\#} f\right|^{2}\left|m^{\prime}(t)\right| d t\right)^{1 / 2} .
\end{aligned}
$$

Now, by [5, Exercise 6.5.2], we have that

$$
\left\|\left(\int_{0}^{\infty}\left|\Delta_{[t, \infty)} \Delta_{\left\lfloor\log _{2} t\right\rfloor}^{\#} f\right|^{2}\left|m^{\prime}(t)\right| d t\right)^{1 / 2}\right\|_{p} \lesssim\left\|\left(\int_{0}^{\infty}\left|\Delta_{\left\lfloor\log _{2} t\right\rfloor}^{\#} f\right|^{2}\left|m^{\prime}(t)\right| d t\right)^{1 / 2}\right\|_{p},
$$


for any $1<p<\infty$, and by hypothesis on $m^{\prime}$,

$$
\begin{aligned}
\left\|\left(\int_{0}^{\infty}\left|\Delta_{\left\lfloor\log _{2} t\right\rfloor}^{\#} f\right|^{2}\left|m^{\prime}(t)\right| d t\right)^{1 / 2}\right\|_{p} & =\left\|\left(\sum_{k \in \mathbb{Z}} \int_{2^{k}}^{2^{k+1}}\left|\Delta_{\left\lfloor\log _{2} t\right\rfloor}^{\#} f\right|^{2}\left|m^{\prime}(t)\right| d t\right)^{1 / 2}\right\|_{p} \\
& =\left\|\left(\sum_{k \in \mathbb{Z}} \int_{2^{k}}^{2^{k+1}}\left|\Delta_{k}^{\#} f\right|^{2}\left|m^{\prime}(t)\right| d t\right)^{1 / 2}\right\|_{p} \\
& =\left\|\left(\sum_{k \in \mathbb{Z}}\left|\Delta_{k}^{\#} f\right|^{2} \int_{2^{k}}^{2^{k+1}}\left|m^{\prime}(t)\right| d t\right)^{1 / 2}\right\|_{p} \\
& \leq A^{1 / 2}\left\|\left(\sum_{k \in \mathbb{Z}}\left|\Delta_{k}^{\#} f\right|^{2}\right)^{1 / 2}\right\|_{p},
\end{aligned}
$$

meaning that

$$
\left\|\left(\sum_{k \in \mathbb{Z}}\left|\left(\widehat{f} m \chi_{k}\right)^{\vee}\right|^{2}\right)^{1 / 2}\right\|_{p} \lesssim\left(\|m\|_{\infty}+A\right)\left\|\left(\sum_{k \in \mathbb{Z}}\left|\Delta_{k}^{\#} f\right|^{2}\right)^{1 / 2}\right\|_{p} .
$$

Finally, we can use Theorem 3.5 with the $L^{p}$ norm in the right hand side of the inequality, so that

$$
\left\|\left(\sum_{k \in \mathbb{Z}}\left|\left(\widehat{f} m \chi_{k}\right)^{\vee}\right|^{2}\right)^{1 / 2}\right\|_{p} \lesssim\|f\|_{p}
$$

and notice that, if we write $\left(\widehat{f} m \chi_{k}\right)^{\vee}=\Delta_{k}^{\#}\left((\widehat{f} m)^{\vee}\right)$, we can use again Theorem 3.5 so that

$$
\left\|(\widehat{f} m)^{\vee}\right\|_{p} \lesssim\left\|\left(\sum_{k \in \mathbb{Z}}\left|\Delta_{k}^{\#}\left((\widehat{f} m)^{\vee}\right)\right|^{2}\right)^{1 / 2}\right\|_{p}=\left\|\left(\sum_{k \in \mathbb{Z}}\left|\left(\widehat{f} m \chi_{k}\right)^{\vee}\right|^{2}\right)^{1 / 2}\right\|_{p},
$$

meaning that

$$
\left\|(\widehat{f} m)^{\vee}\right\|_{p} \lesssim\|f\|_{p}
$$

that is, $m \in M_{p}^{p}$.

We shall now give the $n$-dimensional version of Marcinkiewicz's theorem, for $n \geq 2$. The main hypothesis of the theorem, and ideas used in the proof, are essentially the same. For instance, instead of asking for $C^{1}$ regularity on dyadic intervals, we will ask for differentiability on dyadic sets $I_{\vec{k}}$ as the ones defined in section 3.3. Also, notice that a key ingredient in the proof of the previous theorem was Theorem 3.5. Thus, in this case, we will want to use Theorem 3.7, which is the $n$-dimensional equivalent, $n \geq 2$, of the former. 
Theorem 4.2 (Marcinkiewicz's theorem in $\mathbb{R}^{n}$ ). Let $m: \mathbb{R}^{n} \rightarrow \mathbb{R}$ be a bounded function such that for all $\alpha=\left(\alpha_{1}, \ldots, \alpha_{n}\right)$ with $\left|\alpha_{1}\right|, \ldots,\left|\alpha_{n}\right| \leq 1$, the derivatives $D^{\alpha} m$ are continuous in the interior of $I_{\vec{k}}$, for any $\vec{k} \in \mathbb{Z}^{n}$. Moreover, assume that there exists some constant $A<\infty$ such that for any $\vec{k}=\left(k_{1}, \ldots, k_{n}\right) \in \mathbb{Z}^{n}$, any $I_{\vec{k}}=I_{k_{1}} \times \cdots \times I_{k_{n}}$, and any disjoint partition $\left\{s_{1}, \ldots, s_{j}\right\} \cup\left\{r_{1}, \ldots, r_{l}\right\}=\{1, \ldots, n\}$, it holds

$$
\sup _{\xi_{r_{1}} \in I_{k_{r_{1}}}} \cdots \sup _{\xi_{r_{l}} \in I_{k_{r_{l}}}} \int_{I_{k_{s_{1}}}} \cdots \int_{I_{k_{s_{j}}}}\left|\left(\partial_{s_{1}} \cdots \partial_{s_{j}} m\right)(\xi)\right| d \xi_{s_{j}} \cdots d \xi_{s_{1}} \leq A .
$$

Then, $m \in M_{p}^{p}$ for all $1<p<\infty$.

Proof. We shall do the proof of the theorem for $n=2$, since the higher dimensional cases follow in the same way. As in the proof of Theorem 4.1, we shall assume that $m(\xi)=0$ if $\xi$ lies outside the first quadrant. Let $\vec{k}=\left(k_{1}, k_{2}\right) \in \mathbb{Z}^{2}$. By the fundamental theorem of calculus, we have, for any $\xi=\left(\xi_{1}, \xi_{2}\right)$ with $2^{k_{1}} \leq \xi_{1}<2^{k_{1}+1}$ and $2^{k_{2}} \leq \xi_{2}<2^{k_{2}+1}$, that

$$
\begin{aligned}
m\left(\xi_{1}, \xi_{2}\right)= & m\left(\xi_{1}, 2^{k_{2}}\right)+\int_{2^{k_{2}}}^{\xi_{2}} \partial_{2} m\left(\xi_{1}, t_{2}\right) d t_{2} \\
= & m\left(2^{k_{1}}, 2^{k_{2}}\right)+\int_{2^{k_{1}}}^{\xi_{1}} \partial_{1} m\left(t_{1}, 2^{k_{2}}\right) d t_{1}+\int_{2^{k_{2}}}^{\xi_{2}} \partial_{2} m\left(\xi_{1}, t_{2}\right) d t_{2} \\
= & m\left(2^{k_{1}}, 2^{k_{2}}\right)+\int_{2^{k_{1}}}^{\xi_{1}} \partial_{1} m\left(t_{1}, 2^{k_{2}}\right) d t_{1}+\int_{2^{k_{2}}}^{\xi_{2}} \partial_{2} m\left(2^{k_{1}}, t_{2}\right) d t_{2} \\
& +\int_{2^{k_{1}}}^{\xi_{1}} \int_{2^{k_{2}}}^{\xi_{2}} \partial_{1} \partial_{2} m\left(t_{1}, t_{2}\right) d t_{2} d t_{1} .
\end{aligned}
$$

In particular, for any $f \in \mathcal{S}$,

$$
\begin{aligned}
& \widehat{f}(\xi) m(\xi) \chi_{\vec{k}}(\xi)=m\left(2^{k_{1}}, 2^{k_{2}}\right) \widehat{f}(\xi) \chi_{\vec{k}}(\xi)+\int_{2^{k_{1}}}^{\xi_{1}} \widehat{f}(\xi) \chi_{\vec{k}}(\xi) \partial_{1} m\left(t_{1}, 2^{k_{2}}\right) d t_{1} \\
& \quad+\int_{2^{k_{2}}}^{\xi_{2}} \widehat{f}(\xi) \chi_{\vec{k}}(\xi) \partial_{2} m\left(2^{k_{1}}, t_{2}\right) d t_{2}+\int_{2^{k_{1}}}^{\xi_{1}} \int_{2^{k_{2}}}^{\xi_{2}} \widehat{f}(\xi) \chi_{\vec{k}}(\xi) \partial_{1} \partial_{2} m\left(t_{1}, t_{2}\right) d t_{2} d t_{1} \\
& =m\left(2^{k_{1}}, 2^{k_{2}}\right) \widehat{f}(\xi) \chi_{\vec{k}}(\xi)+\int_{2^{k_{1}}}^{2^{k_{1}+1}} \widehat{f}(\xi) \chi_{\vec{k}}(\xi) \chi_{\left[t_{1}, \infty\right)}\left(\xi_{1}\right) \partial_{1} m\left(t_{1}, 2^{k_{2}}\right) d t_{1} \\
& \quad+\int_{2^{k_{2}}}^{2^{k_{2}+1}} \widehat{f}(\xi) \chi_{\vec{k}}(\xi) \chi_{\left[t_{2}, \infty\right)}\left(\xi_{2}\right) \partial_{2} m\left(2^{k_{1}}, t_{2}\right) d t_{2} \\
& \quad+\int_{2^{k_{1}}}^{2^{k_{1}+1}} \int_{2^{k_{2}}}^{2^{k_{2}+1}} \widehat{f}(\xi) \chi_{\vec{k}}(\xi) \chi_{\left[t_{1}, \infty\right)}\left(\xi_{1}\right) \chi_{\left[t_{2}, \infty\right)}\left(\xi_{2}\right) \partial_{1} \partial_{2} m\left(t_{1}, t_{2}\right) d t_{2} d t_{1},
\end{aligned}
$$

but recall that $\chi_{\vec{k}}(\xi)=\chi_{k_{1}}\left(\xi_{1}\right) \chi_{k_{2}}\left(\xi_{2}\right)$, so 


$$
\begin{aligned}
& \widehat{f}(\xi) m(\xi) \chi_{\vec{k}}(\xi)=m\left(2^{k_{1}}, 2^{k_{2}}\right) \widehat{f}(\xi) \chi_{\vec{k}}(\xi) \\
& \quad+\int_{2^{k_{1}}}^{2^{k_{1}+1}} \widehat{f}(\xi) \chi_{k_{1}}\left(\xi_{1}\right) \chi_{\left[t_{1}, \infty\right)}\left(\xi_{1}\right) \chi_{k_{2}}\left(\xi_{2}\right) \partial_{1} m\left(t_{1}, 2^{k_{2}}\right) d t_{1} \\
& \quad+\int_{2^{k_{2}}}^{2^{k_{2}+1}} \widehat{f}(\xi) \chi_{k_{2}}\left(\xi_{2}\right) \chi_{\left[t_{2}, \infty\right)}\left(\xi_{2}\right) \chi_{k_{1}}\left(\xi_{1}\right) \partial_{2} m\left(2^{k_{1}}, t_{2}\right) d t_{2} \\
& \quad+\int_{2^{k_{1}}}^{2^{k_{1}+1}} \int_{2^{k_{2}}}^{2^{k_{2}+1}} \widehat{f}(\xi) \chi_{k_{2}}\left(\xi_{2}\right) \chi_{\left[t_{2}, \infty\right)}\left(\xi_{2}\right) \chi_{k_{1}}\left(\xi_{1}\right) \chi_{\left[t_{1}, \infty\right)}\left(\xi_{1}\right) \partial_{1} \partial_{2} m\left(t_{1}, t_{2}\right) d t_{2} d t_{1} .
\end{aligned}
$$

Now, taking inverse Fourier transforms leads to

$$
\begin{aligned}
& \left(\widehat{f} m \chi_{\vec{k}}\right)^{\vee}=m\left(2^{k_{1}}, 2^{k_{2}}\right) \Delta_{\vec{k}}^{\#} f+\int_{2^{k_{1}}}^{2^{k_{1}+1}} \Delta_{k_{2}}^{2, \#} \Delta_{\left[t_{1}, \infty\right)} \Delta_{k_{1}}^{1, \#} f \partial_{1} m\left(t_{1}, 2^{k_{2}}\right) d t_{1} \\
& \quad+\int_{2^{k_{2}}}^{2^{k_{2}+1}} \Delta_{k_{1}}^{1, \#} \Delta_{\left[t_{2}, \infty\right)} \Delta_{k_{2}}^{2, \#} f \partial_{2} m\left(2^{k_{1}}, t_{2}\right) d t_{2} \\
& \quad+\int_{2^{k_{1}}}^{2^{k_{1}+1}} \int_{2^{k_{2}}}^{2^{k_{2}+1}} \Delta_{\left[t_{1}, \infty\right)} \Delta_{k_{1}}^{1, \#} \Delta_{\left[t_{2}, \infty\right)} \Delta_{k_{2}}^{2, \#} f \partial_{1} \partial_{2} m\left(t_{1}, t_{2}\right) d t_{2} d t_{1},
\end{aligned}
$$

and hence, in a similar fashion as in the proof of Theorem 4.1, we can take absolute value, apply Cauchy-Schwarz inequality, and use the hypothesis of the theorem, leaving us with

$$
\begin{aligned}
& \left|\left(\widehat{f} m \chi_{\vec{k}}\right)^{\vee}\right| \leq\|m\|_{\infty}\left|\Delta_{\vec{k}}^{\#} f\right|+\int_{2^{k_{1}}}^{2^{k_{1}+1}}\left|\Delta_{k_{2}}^{2, \#} \Delta_{\left[t_{1}, \infty\right)} \Delta_{k_{1}}^{1, \#} f\right|\left|\partial_{1} m\left(t_{1}, 2^{k_{2}}\right)\right| d t_{1} \\
& \quad+\int_{2^{k_{2}}}^{2^{k_{2}+1}}\left|\Delta_{k_{1}}^{1, \#} \Delta_{\left[t_{2}, \infty\right)} \Delta_{k_{2}}^{2, \#} f\right|\left|\partial_{2} m\left(2^{k_{1}}, t_{2}\right)\right| d t_{2} \\
& \quad+\int_{2^{k_{1}}}^{2^{k_{1}+1}} \int_{2^{k_{2}}}^{2^{k_{2}+1}}\left|\Delta_{\left[t_{1}, \infty\right)} \Delta_{k_{1}}^{1, \#} \Delta_{\left[t_{2}, \infty\right)} \Delta_{k_{2}}^{2, \#} f\right|\left|\partial_{1} \partial_{2} m\left(t_{1}, t_{2}\right)\right| d t_{2} d t_{1} \\
& \leq\|m\|_{\infty}\left|\Delta_{\vec{k}}^{\#} f\right|+A^{1 / 2}\left(\int_{2^{k_{1}}}^{2^{k_{1}+1}}\left|\Delta_{k_{2}}^{2, \#} \Delta_{\left[t_{1}, \infty\right)} \Delta_{k_{1}}^{1, \#} f\right|^{2}\left|\partial_{1} m\left(t_{1}, 2^{k_{2}}\right)\right| d t_{1}\right)^{1 / 2} \\
& \quad+A^{1 / 2}\left(\int_{2^{k_{2}}}^{2^{k_{2}+1}}\left|\Delta_{k_{1}}^{1, \#} \Delta_{\left[t_{2}, \infty\right)} \Delta_{k_{2}}^{2, \#} f\right|^{2}\left|\partial_{2} m\left(2^{k_{1}}, t_{2}\right)\right| d t_{2}\right)^{1 / 2} \\
& \quad+A^{1 / 2}\left(\int_{2^{k_{1}}}^{2^{k_{1}+1}} \int_{2^{k_{2}}}^{2^{k_{2}+1}}\left|\Delta_{\left[t_{1}, \infty\right)} \Delta_{k_{1}}^{1, \#} \Delta_{\left[t_{2}, \infty\right)} \Delta_{k_{2}}^{2, \#} f\right|^{2}\left|\partial_{1} \partial_{2} m\left(t_{1}, t_{2}\right)\right| d t_{2} d t_{1}\right)^{1 / 2} .
\end{aligned}
$$


This is valid for any $\vec{k} \in \mathbb{Z}^{2}$, so taking the $\ell^{2}$ sum over all possible $\vec{k}$, we get

$$
\begin{aligned}
\left(\sum_{\vec{k} \in \mathbb{Z}^{2}}\left|\left(\widehat{f} m \chi_{\vec{k}}\right)^{\vee}\right|^{2}\right)^{1 / 2} \lesssim\left(\sum_{\vec{k} \in \mathbb{Z}^{2}}\left|\Delta_{\vec{k}}^{\#} f\right|^{2}\right)^{1 / 2} & +\left(\sum_{\vec{k} \in \mathbb{Z}^{2}} \int_{2^{k_{1}}}^{2^{k_{1}+1}}\left|\Delta_{k_{2}}^{2, \#} \Delta_{\left[t_{1}, \infty\right)} \Delta_{k_{1}}^{1, \#} f\right|^{2}\left|\partial_{1} m\left(t_{1}, 2^{k_{2}}\right)\right| d t_{1}\right)^{1 / 2} \\
& +\left(\sum_{\vec{k} \in \mathbb{Z}^{2}} \int_{2^{k_{2}}}^{2^{k_{2}+1}}\left|\Delta_{k_{1}}^{1, \#} \Delta_{\left[t_{2}, \infty\right)} \Delta_{k_{2}}^{2, \#} f\right|^{2}\left|\partial_{2} m\left(2^{k_{1}}, t_{2}\right)\right| d t_{2}\right)^{1 / 2} \\
& +\left(\sum_{\vec{k} \in \mathbb{Z}^{2}} \int_{2^{k_{1}}}^{2^{k_{1}+1}} \int_{2^{k_{2}}}^{2^{k_{2}+1}}\left|\Delta_{\left[t_{1}, \infty\right)} \Delta_{k_{1}}^{1, \#} \Delta_{\left[t_{2}, \infty\right)} \Delta_{k_{2}}^{2, \#} f\right|^{2}\left|\partial_{1} \partial_{2} m\left(t_{1}, t_{2}\right)\right| d t_{2} d t_{1}\right)^{1 / 2} \\
= & \left(\sum_{\vec{k} \in \mathbb{Z}^{2}}\left|\Delta_{\vec{k}}^{\#} f\right|^{2}\right)^{1 / 2}+\left(\sum_{k_{2} \in \mathbb{Z}} \int_{0}^{\infty}\left|\Delta_{k_{2}}^{2, \#} \Delta_{\left[t_{1}, \infty\right)} \Delta_{\left\lfloor\log _{2} t_{1}\right\rfloor}^{1, \#} f\right|^{2}\left|\partial_{1} m\left(t_{1}, 2^{k_{2}}\right)\right| d t_{1}\right)^{1 / 2} \\
& +\left(\sum_{k_{1} \in \mathbb{Z}} \int_{0}^{\infty}\left|\Delta_{k_{1}}^{1, \#} \Delta_{\left[t_{2}, \infty\right)} \Delta_{\left\lfloor\log _{2} t_{2}\right\rfloor}^{2, \#} f\right|^{2}\left|\partial_{2} m\left(2^{k_{1}}, t_{2}\right)\right| d t_{2}\right)^{1 / 2} \\
& +\left(\int_{0}^{\infty} \int_{0}^{\infty}\left|\Delta_{\left[t_{1}, \infty\right)} \Delta_{\left\lfloor\log _{2} t_{1}\right\rfloor}^{1, \#} \Delta_{\left[t_{2}, \infty\right)} \Delta_{\left\lfloor\log _{2} t_{2}\right\rfloor}^{2, \#} f\right|^{2}\left|\partial_{1} \partial_{2} m\left(t_{1}, t_{2}\right)\right| d t_{2} d t_{1}\right)^{1 / 2} .
\end{aligned}
$$

Now, let $\nu$ be the counting measure given by $\nu(A)=\#\left\{k \in \mathbb{Z}: 2^{k} \in A\right\}$, for any $A \subset(0, \infty)$. In terms of this measure, we have that

$$
\int_{0}^{\infty} g(t) d \nu(t)=\sum_{k \in \mathbb{Z}} \delta_{2^{k}}(g)
$$

where $\delta_{2^{k}}$ is the Dirac delta valuated at $2^{k}$. Hence, we can write the first sum of integrals of the former inequality as

$$
\begin{aligned}
& \sum_{k_{2} \in \mathbb{Z}} \int_{0}^{\infty}\left|\Delta_{k_{2}}^{2, \#} \Delta_{\left[t_{1}, \infty\right)} \Delta_{\left\lfloor\log _{2} t_{1}\right\rfloor}^{1, \#} f\right|^{2}\left|\partial_{1} m\left(t_{1}, 2^{k_{2}}\right)\right| d t_{1} \\
& =\sum_{k_{2} \in \mathbb{Z}} \int_{0}^{\infty}\left|\Delta_{\left\lfloor\log _{2} 2^{\left.k_{2}\right\rfloor}\right.}^{2, \#} \Delta_{\left[t_{1}, \infty\right)} \Delta_{\left\lfloor\log _{2} t_{1}\right\rfloor}^{1, \#} f\right|^{2}\left|\partial_{1} m\left(t_{1}, 2^{\left\lfloor\log _{2} 2^{\left.k_{2}\right\rfloor}\right.}\right)\right| d t_{1} \\
& =\sum_{k_{2} \in \mathbb{Z}} \delta_{2^{k_{2}}}\left(\int_{0}^{\infty}\left|\Delta_{\left\lfloor\log _{2}(\cdot)\right\rfloor}^{2, \#} \Delta_{\left[t_{1}, \infty\right)} \Delta_{\left\lfloor\log _{2} t_{1}\right\rfloor}^{1, \#} f\right|^{2}\left|\partial_{1} m\left(t_{1}, 2^{\left\lfloor\log _{2}(\cdot)\right\rfloor}\right)\right| d t_{1}\right) \\
& =\int_{0}^{\infty} \int_{0}^{\infty}\left|\Delta_{\left\lfloor\log _{2} t_{2}\right\rfloor}^{2, \#} \Delta_{\left[t_{1}, \infty\right)} \Delta_{\left\lfloor\log _{2} t_{1}\right\rfloor}^{1, \#} f\right|^{2}\left|\partial_{1} m\left(t_{1}, 2^{\left\lfloor\log _{2} t_{2}\right\rfloor}\right)\right| d t_{1} d \nu\left(t_{2}\right) \\
& =\int_{0}^{\infty} \int_{0}^{\infty}\left|\Delta_{\left[t_{1}, \infty\right)} \Delta_{\left\lfloor\log _{2} t_{2}\right\rfloor}^{2, \#} \Delta_{\left\lfloor\log _{2} t_{1}\right\rfloor}^{1, \#} f\right|^{2}\left|\partial_{1} m\left(t_{1}, 2^{\left\lfloor\log _{2} t_{2}\right\rfloor}\right)\right| d t_{1} d \nu\left(t_{2}\right),
\end{aligned}
$$


and similarly for the second one, we have

$$
\begin{aligned}
& \sum_{k_{1} \in \mathbb{Z}} \int_{0}^{\infty}\left|\Delta_{k_{1}}^{1, \#} \Delta_{\left[t_{2}, \infty\right)} \Delta_{\left\lfloor\log _{2} t_{2}\right\rfloor}^{2, \#} f\right|^{2}\left|\partial_{2} m\left(2^{k_{1}}, t_{2}\right)\right| d t_{2} \\
& =\int_{0}^{\infty} \int_{0}^{\infty}\left|\Delta_{\left[t_{2}, \infty\right)} \Delta_{\left\lfloor\log _{2} t_{1}\right\rfloor}^{1, \#} \Delta_{\left\lfloor\log _{2} t_{2}\right\rfloor}^{2, \#} f\right|^{2}\left|\partial_{2} m\left(2^{\left\lfloor\log _{2} t_{1}\right\rfloor}, t_{2}\right)\right| d t_{2} d \nu\left(t_{1}\right) .
\end{aligned}
$$

All in all, we have the estimate

$$
\begin{aligned}
& \left(\sum_{\vec{k} \in \mathbb{Z}^{2}}\left|\left(\widehat{f} m \chi_{\vec{k}}\right)^{\vee}\right|^{2}\right)^{1 / 2} \lesssim\left(\sum_{\vec{k} \in \mathbb{Z}^{2}}\left|\Delta_{\vec{k}}^{\#} f\right|^{2}\right)^{1 / 2} \\
& \quad+\left(\int_{0}^{\infty} \int_{0}^{\infty}\left|\Delta_{\left[t_{1}, \infty\right)} \Delta_{\left\lfloor\log _{2} t_{2}\right\rfloor}^{2, \#} \Delta_{\left\lfloor\log _{2} t_{1}\right\rfloor}^{1, \#} f\right|^{2}\left|\partial_{1} m\left(t_{1}, 2^{\left\lfloor\log _{2} t_{2}\right\rfloor}\right)\right| d t_{1} d \nu\left(t_{2}\right)\right)^{1 / 2} \\
& \quad+\left(\int_{0}^{\infty} \int_{0}^{\infty}\left|\Delta_{\left[t_{2}, \infty\right)} \Delta_{\left\lfloor\log _{2} t_{1}\right\rfloor}^{1, \#} \Delta_{\left\lfloor\log _{2} t_{2}\right\rfloor}^{2, \#} f\right|^{2}\left|\partial_{2} m\left(2^{\left\lfloor\log _{2} t_{1}\right\rfloor}, t_{2}\right)\right| d t_{2} d \nu\left(t_{1}\right)\right)^{1 / 2} \\
& \quad+\left(\int_{0}^{\infty} \int_{0}^{\infty}\left|\Delta_{\left[t_{1}, \infty\right)} \Delta_{\left[t_{2}, \infty\right)} \Delta_{\left\lfloor\log _{2} t_{1}\right\rfloor}^{1, \#} \Delta_{\left\lfloor\log _{2} t_{2}\right\rfloor}^{2, \#} f\right|^{2}\left|\partial_{1} \partial_{2} m\left(t_{1}, t_{2}\right)\right| d t_{2} d t_{1}\right)^{1 / 2},
\end{aligned}
$$

and if we take $L^{p}$ norms and use [5, Exercise 6.5.2], we obtain

$$
\begin{aligned}
& \left\|\left(\sum_{\vec{k} \in \mathbb{Z}^{2}}\left|\left(\widehat{f} m \chi_{\vec{k}}\right)^{\vee}\right|^{2}\right)^{1 / 2}\right\|\left\|_{p} \lesssim\right\|\left(\sum_{\vec{k} \in \mathbb{Z}^{2}}\left|\Delta_{\vec{k}}^{\#} f\right|^{2}\right)^{1 / 2} \|_{p} \\
& +\left\|\left(\int_{0}^{\infty} \int_{0}^{\infty}\left|\Delta_{\left\lfloor\log _{2} t_{2}\right\rfloor}^{2, \#} \Delta_{\left\lfloor\log _{2} t_{1}\right\rfloor}^{1, \#} f\right|^{2}\left|\partial_{1} m\left(t_{1}, 2^{\left\lfloor\log _{2} t_{2}\right\rfloor}\right)\right| d t_{1} d \nu\left(t_{2}\right)\right)^{1 / 2}\right\|_{p} \\
& +\left\|\left(\int_{0}^{\infty} \int_{0}^{\infty}\left|\Delta_{\left\lfloor\log _{2} t_{1}\right\rfloor}^{1, \#} \Delta_{\left\lfloor\log _{2} t_{2}\right\rfloor}^{2, \#} f\right|^{2}\left|\partial_{2} m\left(2^{\left\lfloor\log _{2} t_{1}\right\rfloor}, t_{2}\right)\right| d t_{2} d \nu\left(t_{1}\right)\right)^{1 / 2}\right\|_{p} \\
& +\left\|\left(\int_{0}^{\infty} \int_{0}^{\infty}\left|\Delta_{\left\lfloor\log _{2} t_{1}\right\rfloor}^{1, \#} \Delta_{\left\lfloor\log _{2} t_{2}\right\rfloor}^{2, \#} f\right|^{2}\left|\partial_{1} \partial_{2} m\left(t_{1}, t_{2}\right)\right| d t_{2} d t_{1}\right)^{1 / 2}\right\|_{p}
\end{aligned}
$$

Finally, we can use again our hypothesis, and get 


$$
\begin{aligned}
& \left\|\left(\sum_{\vec{k} \in \mathbb{Z}^{2}}\left|\left(\widehat{f} m \chi_{\vec{k}}\right)^{\vee}\right|^{2}\right)^{1 / 2}\right\|_{p} \lesssim\left\|\left(\sum_{\vec{k} \in \mathbb{Z}^{2}}\left|\Delta_{\vec{k}}^{\#} f\right|^{2}\right)^{1 / 2}\right\|_{p} \\
& +\left\|\left(\sum_{k_{2} \in \mathbb{Z}} \int_{0}^{\infty}\left|\Delta_{k_{2}}^{2, \#} \Delta_{\left\lfloor\log _{2} t_{1}\right\rfloor}^{1, \#} f\right|^{2}\left|\partial_{1} m\left(t_{1}, 2^{k_{2}}\right)\right| d t_{1}\right)^{1 / 2}\right\|_{p} \\
& +\left\|\left(\sum_{k_{1} \in \mathbb{Z}} \int_{0}^{\infty}\left|\Delta_{k_{1}}^{1, \#} \Delta_{\left\lfloor\log _{2} t_{2}\right\rfloor}^{2, \#} f\right|^{2}\left|\partial_{2} m\left(2^{k_{1}}, t_{2}\right)\right| d t_{2}\right)^{1 / 2}\right\|_{p} \\
& +\left\|\left(\int_{0}^{\infty} \int_{0}^{\infty}\left|\Delta_{\left\lfloor\log _{2} t_{1}\right\rfloor}^{1, \#} \Delta_{\left\lfloor\log _{2} t_{2}\right\rfloor}^{2, \#} f\right|^{2}\left|\partial_{1} \partial_{2} m\left(t_{1}, t_{2}\right)\right| d t_{2} d t_{1}\right)^{1 / 2}\right\|_{p} \\
& =\left\|\left(\sum_{\vec{k} \in \mathbb{Z}^{2}}\left|\Delta_{\vec{k}}^{\#} f\right|^{2}\right)^{1 / 2}\right\|_{p}+\left\|\left(\sum_{\vec{k} \in \mathbb{Z}^{2}} \int_{2^{k_{1}}}^{2^{k_{1}+1}}\left|\Delta_{k_{2}}^{2, \#} \Delta_{k_{1}}^{1, \#} f\right|^{2}\left|\partial_{1} m\left(t_{1}, 2^{k_{2}}\right)\right| d t_{1}\right)^{1 / 2}\right\|_{p} \\
& +\left\|\left(\sum_{\vec{k} \in \mathbb{Z}^{2}} \int_{2^{k_{2}}}^{2^{k_{2}+1}}\left|\Delta_{k_{1}}^{1, \#} \Delta_{k_{2}}^{2, \#} f\right|^{2}\left|\partial_{2} m\left(2^{k_{1}}, t_{2}\right)\right| d t_{2}\right)^{1 / 2}\right\|_{p} \\
& +\left\|\left(\sum_{\vec{k} \in \mathbb{Z}^{2}} \int_{2^{k_{1}}}^{2^{k_{1}+1}} \int_{2^{k_{2}}}^{2^{k_{2}+1}}\left|\Delta_{k_{1}}^{1, \#} \Delta_{k_{2}}^{2, \#} f\right|^{2}\left|\partial_{1} \partial_{2} m\left(t_{1}, t_{2}\right)\right| d t_{2} d t_{1}\right)^{1 / 2}\right\|_{p} \\
& =\left\|\left(\sum_{\vec{k} \in \mathbb{Z}^{2}}\left|\Delta_{\vec{k}}^{\#} f\right|^{2}\right)^{1 / 2}\right\|_{p}+\left\|\left(\sum_{\vec{k} \in \mathbb{Z}^{2}}\left|\Delta_{\vec{k}}^{\#} f\right|^{2} \int_{2^{k_{1}}}^{2^{k_{1}+1}}\left|\partial_{1} m\left(t_{1}, 2^{k_{2}}\right)\right| d t_{1}\right)^{1 / 2}\right\|_{p} \\
& +\left\|\left(\sum_{\vec{k} \in \mathbb{Z}^{2}}\left|\Delta_{\vec{k}}^{\#} f\right|^{2} \int_{2^{k_{2}}}^{2^{k_{2}+1}}\left|\partial_{2} m\left(2^{k_{1}}, t_{2}\right)\right| d t_{2}\right)^{1 / 2}\right\|_{p} \\
& +\left\|\left(\sum_{\vec{k} \in \mathbb{Z}^{2}}\left|\Delta_{\vec{k}}^{\#} f\right|^{2} \int_{2^{k_{1}}}^{2^{k_{1}+1}} \int_{2^{k_{2}}}^{2^{k_{2}+1}}\left|\partial_{1} \partial_{2} m\left(t_{1}, t_{2}\right)\right| d t_{2} d t_{1}\right)^{1 / 2}\right\|_{p} \\
& \lesssim\left\|\left(\sum_{\vec{k} \in \mathbb{Z}^{2}}\left|\Delta_{\vec{k}}^{\#} f\right|^{2}\right)^{1 / 2}\right\|_{p}
\end{aligned}
$$

from which Theorem 3.7 gives us that

$$
\left\|\left(\sum_{\vec{k} \in \mathbb{Z}^{2}}\left|\left(\widehat{f} m \chi_{\vec{k}}\right)^{\vee}\right|^{2}\right)^{1 / 2}\right\|_{p} \lesssim\|f\|_{p} .
$$

But writing $\left(\widehat{f} m \chi_{\vec{k}}\right)^{\vee}=\Delta_{\vec{k}}^{\#}\left((\widehat{f} m)^{\vee}\right)$ and using again Theorem 3.7, we obtain

$$
\left\|\left((\widehat{f} m)^{\vee}\right)\right\|_{p} \lesssim\left\|\left(\sum_{\vec{k} \in \mathbb{Z}^{2}}\left|\left(\widehat{f} m \chi_{\vec{k}}\right)^{\vee}\right|^{2}\right)^{1 / 2}\right\|_{p}
$$

so in the end,

$$
\left\|(\widehat{f} m)^{\vee}\right\|_{p} \lesssim\|f\|_{p}
$$

as we wanted. 
The growth condition on Marcinkiewicz's theorem might be a little complicated in order to actually check it in particular cases. We give now a stronger condition that allow easier calculations.

Corollary 4.3. Let $m$ be a bounded, n-times continuously differentiable function, defined away from the coordinate axes. Assume that there exists some constant $A<\infty$ such that for any disjoint partition $\left\{s_{1}, \ldots, s_{j}\right\} \cup\left\{r_{1}, \ldots, r_{l}\right\}=\{1, \ldots, n\}$, it holds

$$
\sup _{\xi_{r_{1}} \in \mathbb{R} \backslash\{0\}} \cdots \sup _{\xi_{r_{l}} \in \mathbb{R} \backslash\{0\}}\left|\left(\partial_{s_{1}} \cdots \partial_{s_{j}} m\right)(\xi)\right| \leq A\left|\xi_{s_{1}}\right|^{-1} \cdots\left|\xi_{s_{j}}\right|^{-1} .
$$

Then, $m \in M_{p}^{p}$ for any $1<p<\infty$.

Proof. We simply check the growth condition on Marcinkiewicz's theorem. Indeed,

$$
\begin{aligned}
& \int_{I_{k_{s_{1}}}} \cdots \int_{I_{k_{s_{j}}}}\left|\left(\partial_{s_{1}} \cdots \partial_{s_{j}} m\right)(\xi)\right| d \xi_{s_{j}} \cdots d \xi_{s_{1}} \\
& \leq A \int_{I_{k_{s_{1}}}} \cdots \int_{I_{k_{s_{j}}}}\left|\xi_{s_{1}}\right|^{-1} \cdots\left|\xi_{s_{j}}\right|^{-1} d \xi_{s_{j}} \cdots d \xi_{s_{1}} \\
& =A \int_{I_{k_{s_{1}}}}\left|\xi_{s_{1}}\right|^{-1} d \xi_{s_{1}} \cdots \int_{I_{k_{s_{j}}}}\left|\xi_{s_{j}}\right|^{-1} d \xi_{s_{j}},
\end{aligned}
$$

and all of the integrals that appear here are just integrals of the function $f(t)=1 / t$ on dyadic sets of the form $\left(-2^{u+1},-2^{u}\right] \cup\left[2^{u}, 2^{u+1}\right)$, for which one has

$$
\int_{\left(-2^{u+1},-2^{u}\right] \cup\left[2^{u}, 2^{u+1}\right)} \frac{1}{t} d t=2 \int_{2^{u}}^{2^{u+1}} \frac{1}{t} d t=2\left(\log 2^{u+1}-\log 2^{u}\right)=2 \log 2 .
$$

Thus, the result follows taking $A^{\prime}=A(2 \log 2)^{n}$.

Example 4.4. Let $m$ be a $C^{\infty}$ function on $\mathbb{R}^{n} \backslash\{0\}$, for which there exists $k_{1}, \ldots, k_{n} \in(0,+\infty)$ and $s \in \mathbb{R}$ such that

$$
m\left(\lambda^{k_{1}} \xi_{1}, \ldots, \lambda^{k_{n}} \xi_{n}\right)=\lambda^{i s} m\left(\xi_{1}, \ldots, \xi_{n}\right), \forall \xi \in \mathbb{R}^{n}, \forall \lambda>0 .
$$

We see that, in this case, $m \in M_{p}^{p}$ for any $1<p<\infty$. Indeed, we pick $\alpha \in \mathbb{N}^{n}$, and we differentiate in the previous equality to get

$$
\lambda^{k_{1} \alpha_{1}+\cdots k_{n} \alpha_{n}} \partial^{\alpha} m\left(\lambda^{k_{1}} \xi_{1}, \ldots, \lambda^{k_{n}} \xi_{n}\right)=\lambda^{i s} \partial^{\alpha} m\left(\xi_{1}, \ldots, \xi_{n}\right) .
$$

Now, given $\xi \in \mathbb{R}^{n} \backslash\{0\}$, there exists a unique $\lambda_{\xi}>0$ so that $\left(\lambda_{\xi}^{k_{1}} \xi_{1}, \ldots, \lambda_{\xi}^{k_{n}} \xi_{n}\right)$ is in the unit sphere $S^{n-1}=\left\{\xi \in \mathbb{R}^{n}:|\xi|=1\right\}$. This is so because $\psi(\lambda)=$ $\xi_{1}^{2} \lambda^{2 k_{1}}+\cdots+\xi_{n}^{2} \lambda^{2 k_{n}}-1$ is a continuous, increasing function of $\lambda$, such that $\psi(0)=-1$, and $\lim _{\lambda \rightarrow \infty} \psi(\lambda)=\infty$. Notice that, with this $\lambda_{\xi}$, one has that $\left|\lambda^{k_{j}} \xi_{j}\right| \leq 1$, so $\lambda^{k_{j}} \leq\left|\xi_{j}\right|^{-1}$. Therefore,

$$
\begin{aligned}
\left|\partial^{\alpha} m\left(\xi_{1}, \ldots, \xi_{n}\right)\right| & =\left|\lambda_{\xi}^{k_{1} \alpha_{1}+\cdots k_{n} \alpha_{n}} \partial^{\alpha} m\left(\lambda_{\xi}^{k_{1}} \xi_{1}, \ldots, \lambda_{\xi}^{k_{n}} \xi_{n}\right)\right| \\
& \leq \sup _{\xi \in S^{n-1}}\left|\partial^{\alpha} m(\xi)\right|\left(\lambda^{k_{1}}\right)^{\alpha_{1}} \cdots\left(\lambda^{k_{n}}\right)^{\alpha_{n}} \\
\leq C_{\alpha}\left|\xi_{1}\right|^{-\alpha_{1}} \cdots\left|\xi_{n}\right|^{-\alpha_{n}} &
\end{aligned}
$$

and so Corollary 4.3 can be applied to get the result. 


\subsection{Mihlin-Hörmander's theorem}

Like Marcinkiewicz's theorem, Mihlin-Hörmander's theorem allow us to see if a certain bounded function is an $M_{p}^{p}$ multiplier. In this result, we allow our function to have a less restrictive growth of its derivatives. The price to pay, though, is that the function must have derivatives of higher order.

Theorem 4.5 (Mihlin-Hörmander's theorem). Let $m$ be a bounded function on $\mathbb{R}^{n} \backslash\{0\}$ such that for some $A<\infty$,

$$
\left(\int_{R<|\xi|<2 R}\left|D^{\alpha} m(\xi)\right|^{2} d \xi\right)^{1 / 2} \leq A R^{n / 2-|\alpha|}<\infty
$$

whenever $|\alpha| \leq n_{0}:=\lfloor n / 2\rfloor+1$ and $R>0$. Then, $m \in M_{p}^{p}$ for any $1<p<\infty$.

Proof. Let $\widehat{\psi} \in \mathcal{S}$ be supported in the annulus $1 / 2 \leq|\xi| \leq 2$ with

$$
\sum_{k \in \mathbb{Z}} \widehat{\psi_{2^{-k}}}(\xi)=\sum_{k \in \mathbb{Z}} \widehat{\psi}\left(2^{-k} \xi\right)=1, \forall \xi \neq 0
$$

Let $m_{k}(\xi)=m(\xi) \widehat{\psi}\left(2^{-k} \xi\right)$, for any $k \in \mathbb{Z}$, and define $K_{k}=\widetilde{m}_{k}{ }^{1}$. In general, $K_{k}$ would be a distribution, but observe that $m_{k} \in L^{2}$, so $K_{k}$ is actually an $L^{2}$ function. Trivially, $\sum_{k \in \mathbb{Z}} K_{k}=K$ in $\mathcal{S}^{\prime}$. Indeed, notice that

$$
K_{k}(f)=\left(\left(\widehat{\tilde{f}} m_{k}\right)^{\vee}\right)(0)=\int_{\mathbb{R}^{n}} m_{k}(\xi) \widehat{\tilde{f}}(\xi) d \xi
$$

as well as

$$
K(f)=\left((\widehat{\tilde{f}} m)^{\vee}\right)(0)=\int_{\mathbb{R}^{n}} m(\xi) \widehat{\tilde{f}}(\xi) d \xi
$$

so, by the dominated convergence theorem, we have

$$
\begin{aligned}
\left(\sum_{|k| \leq N} K_{k}\right)(f) & =\sum_{|k| \leq N} K_{k}(f)=\sum_{|k| \leq N}\left(\left(\widehat{\tilde{f}} m_{k}\right)^{\vee}\right)(0) \\
& =\left(\left(\widehat{\tilde{f}} \sum_{|k| \leq N} m_{k}\right)^{\vee}\right)(0)= \\
& =\left(\left(\widehat{\tilde{f}} m \sum_{|k| \leq N} \widehat{\psi_{2^{-k}}}\right)^{\vee}\right)(0) \stackrel{N \rightarrow \infty}{\longrightarrow}\left((\widehat{\tilde{f}} m)^{\vee}\right)(0)=K(f) .
\end{aligned}
$$

This is true for any function $f \in \mathcal{S}$, so by Banach-Steinhaus theorem [1, Theorem 3.15], we have the desired convergence. Next, we shall see that there

\footnotetext{
${ }^{1}$ Intuitively, $K_{k}$ is the part of the distribution $K=\check{m}$ whose frequencies are located in the annulus $2^{k-1} \leq|\xi| \leq 2^{k+1}$
} 
exists some constant $C$ such that

$$
\begin{array}{r}
\sup _{k \in \mathbb{Z}} \int_{\mathbb{R}^{n}}\left|K_{k}(x)\right|\left(1+2^{k}|x|\right)^{1 / 4} d x \leq C \\
\sup _{k \in \mathbb{Z}} \frac{1}{2^{k}} \int_{\mathbb{R}^{n}}\left|\nabla K_{k}(x)\right|\left(1+2^{k}|x|\right)^{1 / 4} d x \leq C .
\end{array}
$$

For the first inequality, we write

$$
\int_{\mathbb{R}^{n}}\left|K_{k}(x)\right|\left(1+2^{k}|x|\right)^{1 / 4} d x=\int_{\mathbb{R}^{n}}\left|K_{k}(x)\right|\left(1+2^{k}|x|\right)^{n_{0}}\left(1+2^{k}|x|\right)^{1 / 4-n_{0}} d x .
$$

Notice that the integrand is positive. Thus, by Cauchy-Schwarz inequality applied to $\left|K_{k}(x)\right|\left(1+2^{k}|x|\right)^{n_{0}}$ and $\left(1+2^{k}|x|\right)^{1 / 4-n_{0}}$, we have

$$
\begin{aligned}
& \int_{\mathbb{R}^{n}}\left|K_{k}(x)\right|\left(1+2^{k}|x|\right)^{1 / 4} d x \\
& \leq\left(\int_{\mathbb{R}^{n}}\left|K_{k}(x)\right|^{2}\left(1+2^{k}|x|\right)^{2 n_{0}} d x\right)^{1 / 2}\left(\int_{\mathbb{R}^{n}}\left(1+2^{k}|x|\right)^{1 / 2-2 n_{0}} d x\right)^{1 / 2} .
\end{aligned}
$$

For the first integral, notice that

$$
\left(1+2^{k}|x|\right)^{n_{0}} \lesssim \sum_{j=0}^{n_{0}} 2^{j k}|x|^{j},
$$

but we have

$$
|x|^{j}=\sqrt{\left(x_{1}^{2}+\cdots+x_{n}^{2}\right)^{k}} \leq \max _{1 \leq l \leq n}\left|x_{l}\right|^{j}=\left|x^{\gamma_{x}}\right|,
$$

where $\gamma_{x}$ is some multiindex with $\left|\gamma_{x}\right|=k$, so clearly

$$
\sum_{j=0}^{n_{0}} 2^{j k}|x|^{j} \leq \sum_{|\gamma| \leq n_{0}} 2^{k|\gamma|}\left|x^{\gamma}\right| .
$$

For the second integral, we easily have that

$$
2 n_{0}-1 / 2=2\lfloor n / 2\rfloor+3 / 2>n,
$$

for any $n \in \mathbb{N}$, so $2 n_{0}-1 / 2-(n-1)=\beta>1$, meaning that

$$
\begin{aligned}
\left(\int_{\mathbb{R}^{n}}\left(1+2^{k}|x|\right)^{1 / 2-2 n_{0}} d x\right)^{1 / 2} & =\frac{1}{2^{(k / 2)\left(2 n_{0}-1 / 2\right)}}\left(\int_{\mathbb{R}^{n}}\left(2^{-k}+|x|\right)^{1 / 2-2 n_{0}} d x\right)^{1 / 2} \\
& \lesssim \frac{1}{2^{(k / 2)\left(2 n_{0}-1 / 2\right)}}\left(\int_{0}^{\infty} \frac{1}{\left(2^{-k}+r\right)^{2 n_{0}-1 / 2}} r^{n-1} d r\right)^{1 / 2} \\
& \lesssim \frac{1}{2^{(k / 2)\left(2 n_{0}-1 / 2\right)}} \\
& \leq \frac{1}{2^{n k / 2}}
\end{aligned}
$$


Therefore, we have that

$$
\begin{aligned}
\int_{\mathbb{R}^{n}}\left|K_{k}(x)\right|\left(1+2^{k}|x|\right)^{1 / 4} d x & \lesssim \frac{1}{2^{n k / 2}}\left(\int_{\mathbb{R}^{n}}\left|K_{k}(x)\right|^{2}\left|\sum_{|\gamma| \leq n_{0}} 2^{k|\gamma|}\right| x^{\gamma}||^{2} d x\right)^{1 / 2} \\
& \leq \frac{1}{2^{n k / 2}} \sum_{|\gamma| \leq n_{0}}\left(\int_{\mathbb{R}^{n}}\left|K_{k}(x)\right|^{2} 2^{2 k|\gamma|}\left|x^{\gamma}\right|^{2} d x\right)^{1 / 2} \\
& =\frac{1}{2^{n k / 2}} \sum_{|\gamma| \leq n_{0}} 2^{k|\gamma|}\left(\int_{\mathbb{R}^{n}}\left|x^{\gamma} K_{k}(x)\right|^{2} d x\right)^{1 / 2}
\end{aligned}
$$

and since $\widehat{x^{\gamma} K_{k}}=C_{\gamma} D^{\gamma} \widehat{K_{k}}=C_{\gamma} D^{\gamma} m_{k}$, we have by Plancherel's theorem that

$$
\int_{\mathbb{R}^{n}}\left|K_{k}(x)\right|\left(1+2^{k}|x|\right)^{1 / 4} d x \lesssim \frac{1}{2^{n k / 2}} \sum_{|\gamma| \leq n_{0}} 2^{k|\gamma|}\left(\int_{\mathbb{R}^{n}}\left|D^{\gamma} m_{k}(\xi)\right|^{2} d \xi\right)^{1 / 2} .
$$

We now use Leibniz's rule to write $D^{\gamma} m_{k}$ as

$$
\begin{aligned}
& D^{\gamma} m_{k}(\xi)=D^{\gamma}\left(m \widehat{\psi_{2^{-k}}}\right)(\xi)=\sum_{\delta \leq \gamma} C_{\delta, \gamma} D^{\delta} m(\xi) D^{\gamma-\delta}\left(\widehat{\psi_{2^{-k}}}(\xi)\right) \\
& =\sum_{\delta \leq \gamma} C_{\delta, \gamma} D^{\delta} m(\xi) D^{\gamma-\delta}\left(\widehat{\psi}\left(2^{-k} \xi\right)\right) \\
& =\sum_{\delta \leq \gamma} C_{\delta, \gamma} D^{\delta} m(\xi) D^{\gamma-\delta} \widehat{\psi}\left(2^{-k} \xi\right) 2^{-k|\gamma-\delta|},
\end{aligned}
$$

and so we finally have

$$
\begin{aligned}
& \int_{\mathbb{R}^{n}}\left|K_{k}(x)\right|\left(1+2^{k}|x|\right)^{1 / 4} d x \\
& =\frac{1}{2^{n k / 2}} \sum_{|\gamma| \leq n_{0}} 2^{k|\gamma|}\left(\int_{\mathbb{R}^{n}}\left|\sum_{\delta \leq \gamma} C_{\delta, \gamma} D^{\delta} m(\xi) D^{\gamma-\delta} \widehat{\psi}\left(2^{-k} \xi\right) 2^{-k|\gamma-\delta|}\right|^{2} d \xi\right)^{1 / 2} \\
& \lesssim \frac{1}{2^{n k / 2}} \sum_{|\gamma| \leq n_{0}} 2^{k|\gamma|} \sum_{\delta \leq \gamma} 2^{-k|\gamma-\delta|}\left(\int_{\mathbb{R}^{n}}\left|D^{\delta} m(\xi) D^{\gamma-\delta} \widehat{\psi}\left(2^{-k} \xi\right)\right|^{2} d \xi\right)^{1 / 2} \\
& \left.\leq \frac{1}{2^{n k / 2}} \sum_{|\gamma| \leq n_{0}} \sum_{\delta \leq \gamma} 2^{k|\delta|}\left(\int_{2^{k-1} \leq|\xi| \leq 2^{k+1}}\left|D^{\delta} m(\xi)\right|^{2} d \xi\right)^{1 / 2}\left|D^{\delta} m(\xi)\right|^{2} d \xi+\int_{2^{k} \leq|\xi| \leq 2^{k+1}}\left|D^{\delta} m(\xi)\right|^{2} d \xi\right)^{1 / 2} \\
& =\frac{1}{2^{n k / 2}} \sum_{|\gamma| \leq n_{0}} \sum_{\delta \leq \gamma} 2^{k|\delta|}\left(\int_{2^{k-1} \leq|\xi| \leq 2^{k}}\right. \\
& \leq \frac{1}{2^{n k / 2}} \sum_{|\gamma| \leq n_{0}} \sum_{\delta \leq \gamma} 2^{k|\delta|}\left(A^{2} 2^{2(k-1)(n / 2-|\delta|)}+A^{2} 2^{2 k(n / 2-|\delta|)}\right)^{1 / 2} \\
& \leq \frac{\sqrt{2}}{2^{n k / 2}} \sum_{|\gamma| \leq n_{0}} \sum_{\delta \leq \gamma} 2^{k|\delta|} A 2^{k(n / 2-|\delta|)}=\frac{\sqrt{2}}{2^{n k / 2}} \sum_{|\gamma| \leq n_{0}} \sum_{\delta \leq \gamma} A 2^{n k / 2}:=C .
\end{aligned}
$$


Similarly, to prove inequality (4.2), we can repeat the same argument for each derivative $\partial_{x_{i}} K_{k}$. As before, we shall arrive to

$$
\frac{1}{2^{k}} \int_{\mathbb{R}^{n}}\left|\partial_{x_{i}} K_{k}(x)\right|\left(1+2^{k}|x|\right)^{1 / 4} d x \lesssim \frac{1}{2^{n k / 2}} \sum_{|\gamma| \leq n_{0}} 2^{k|\gamma|}\left(\int_{\mathbb{R}^{n}}\left|\frac{1}{2^{k}} x^{\gamma} \partial_{x_{i}} K_{k}(x)\right|^{2} d x\right)^{1 / 2} .
$$

Now, since $\widehat{x^{\gamma} \partial_{x_{i}} K} k(\xi)=C_{\gamma, i} D^{\gamma}\left(\xi_{i} m_{k}(\xi)\right)$, using Plancherel's theorem we get

$\frac{1}{2^{k}} \int_{\mathbb{R}^{n}}\left|\partial_{x_{i}} K_{k}(x)\right|\left(1+2^{k}|x|\right)^{1 / 4} d x \lesssim \frac{1}{2^{n k / 2}} \sum_{|\gamma| \leq n_{0}} 2^{k|\gamma|}\left(\int_{\mathbb{R}^{n}}\left|\frac{1}{2^{k}} D^{\gamma}\left(\xi_{i} m_{k}(\xi)\right)\right|^{2} d \xi\right)^{1 / 2}$,

and if we write

$$
\frac{1}{2^{k}} D^{\gamma}\left(\xi_{i} m_{k}(\xi)\right)=\frac{1}{2^{k}} D^{\gamma}\left(\xi_{i} m(\xi) \widehat{\psi}\left(2^{-k} \xi\right)\right):=D^{\gamma}\left(m(\xi) \widehat{\psi}_{i}\left(2^{-k} \xi\right)\right),
$$

where $\widehat{\psi}_{i}(\xi)=\xi_{i} \widehat{\psi}(\xi)$, notice that $\widehat{\psi}_{i}$ is also supported in $1 / 2 \leq|\xi| \leq 2$, and so repeating the previous computation using Leibniz's rule will give us the desired bound.

With this inequalities proven, we shall now use (4.1) to see that $K$ is actually a real valued function defined on $\mathbb{R}^{n} \backslash\{0\}$; that is,

$$
K(x)=\sum_{k \in \mathbb{Z}} K_{k}(x)
$$

converges to a finite value for almost any $x \in \mathbb{R}^{n} \backslash\{0\}$. To begin with, we have that the series

$$
\sum_{k \geq 0}\left|K_{k}(x)\right|
$$

is of positive terms for each $x \in \mathbb{R}^{n} \backslash\{0\}$, so it converges either to a finite or to an infinite value. Because of (4.1), it follows that, for $\delta>0$,

$$
\left(1+2^{k} \delta\right)^{1 / 4} \int_{|x|>\delta}\left|K_{k}(x)\right| d x \leq \int_{|x|>\delta}\left|K_{k}(x)\right|\left(1+2^{k}|x|\right)^{1 / 4} d x \leq C,
$$

so, by the monotone convergence theorem,

$$
\int_{|x|>\delta} \sum_{k \geq 0}\left|K_{k}(x)\right| d x \leq \sum_{k \geq 0} \frac{C}{\left(1+2^{k} \delta\right)^{1 / 4}}:=\tilde{C}<\infty .
$$

This means that the set $\left\{|x|>\delta: \sum_{k \geq 0}\left|K_{k}(x)\right|=\infty\right\}$ is of measure zero, for any $\delta>0$, so $\sum_{k \geq 0}\left|K_{k}(x)\right|<\infty$ for almost every $x \in \mathbb{R}^{n} \backslash\{0\}$. On the other hand, notice that

$$
\begin{aligned}
\sup _{k \in \mathbb{Z}} \int_{\mathbb{R}^{n}}\left|K_{k}(x)\right|\left(1+2^{k}|x|\right)^{-1 / 4} d x & =\sup _{k \in \mathbb{Z}} \int_{\mathbb{R}^{n}}\left|K_{k}(x)\right|\left(1+2^{k}|x|\right)^{1 / 4}\left(1+2^{k}|x|\right)^{-1 / 8} d x \\
& \leq \sup _{k \in \mathbb{Z}} \int_{\mathbb{R}^{n}}\left|K_{k}(x)\right|\left(1+2^{k}|x|\right)^{1 / 4} d x \leq C,
\end{aligned}
$$


which means that

$$
\left(1+2^{k} 2 \delta\right)^{-1 / 4} \int_{|x|<2 \delta}\left|K_{k}(x)\right| d x \leq \int_{|x|<2 \delta}\left|K_{k}(x)\right|\left(1+2^{k}|x|\right)^{-1 / 4} d x \leq C,
$$

and so

$$
\int_{\delta<|x|<2 \delta} \sum_{k<0}\left|K_{k}(x)\right| d x \leq C \sum_{k<0}\left(1+2^{k} 2 \delta\right)^{1 / 4}-\left(1+2^{k} \delta\right)^{1 / 4}:=\tilde{\widetilde{C}}<\infty,
$$

which yields that for any $\delta>0$, the set $\left\{\delta<|x|<2 \delta: \sum_{k<0}\left|K_{k}(x)\right|=\infty\right\}$ is of measure zero, and in particular, $\sum_{k<0}\left|K_{k}(x)\right|<\infty$ for almost every $x \in \mathbb{R}^{n} \backslash\{0\}$. All in all, we conclude that

$$
\sum_{k \in \mathbb{Z}}\left|K_{k}(x)\right|<\infty
$$

for almost any $x \in \mathbb{R}^{n} \backslash\{0\}$, and hence

$$
\sum_{k \in \mathbb{Z}} K_{k}(x)
$$

converges to a finite value for almost any $x \in \mathbb{R}^{n} \backslash\{0\}$.

In order to conclude the proof, we are going to see that $K$ satisfies Theorem 1.5. Both the first and third conditions of the theorem are already seen. Also, $T$ is bounded from $L^{2}$ to $L^{2}$ because $m \in L^{\infty}$. Thus, it only remains to see that

$$
\sup _{y \neq 0} \int_{|x| \geq 2|y|}|K(x-y)-K(x)| d x \leq C^{\prime}<\infty .
$$

Instead, a stronger bound, namely,

$$
\sum_{k \in \mathbb{Z}} \int_{|x| \geq 2|y|}\left|K_{k}(x-y)-K_{k}(x)\right| d x \leq C^{\prime}<\infty,
$$

for every $y \neq 0$. Fix such $y$ and take $k_{0} \in \mathbb{Z}$ so that $2^{-k_{0}} \leq|y| \leq 2^{-k_{0}+1}$. For $k>k_{0}$,

$$
\begin{aligned}
& \sum_{k>k_{0}} \int_{|x| \geq 2|y|}\left|K_{k}(x-y)-K_{k}(x)\right| d x \leq \sum_{k>k_{0}} \int_{|x| \geq 2|y|}\left|K_{k}(x-y)\right|+\left|K_{k}(x)\right| d x \\
& \leq \sum_{k>k_{0}}\left(\int_{|x| \geq 2|y|}\left|K_{k}(x-y)\right| d x+\int_{|x| \geq 2|y|}\left|K_{k}(x)\right| d x\right) \\
& \leq \sum_{k>k_{0}}\left(\int_{|x| \geq|y|}\left|K_{k}(x)\right| d x+\int_{|x| \geq 2|y|}\left|K_{k}(x)\right| d x\right) \\
& \leq 2 \sum_{k>k_{0}} \int_{|x| \geq|y|}\left|K_{k}(x)\right| d x=2 \sum_{k>k_{0}} \int_{|x| \geq|y|}\left|K_{k}(x)\right| \frac{\left(1+2^{k}|x|\right)^{1 / 4}}{\left(1+2^{k}|x|\right)^{1 / 4}} d x \\
& \leq 2 \sum_{k>k_{0}} \frac{1}{\left(1+2^{k}|y|\right)^{1 / 4}} \int_{\mathbb{R}^{n}}\left|K_{k}(x)\right|\left(1+2^{k}|x|\right)^{1 / 4} d x \leq C^{\prime},
\end{aligned}
$$


where we use (4.1) for the last inequality and the fact that $\sum_{k>k_{0}} 1 /\left(1+2^{k}|y|\right)^{1 / 4}$ converges. On the other hand, for $k \leq k_{0}$,

$$
\begin{aligned}
& \sum_{k \leq k_{0}} \int_{|x| \geq 2|y|}\left|K_{k}(x-y)-K_{k}(x)\right| d x \leq \sum_{k \leq k_{0}} \int_{|x| \geq 2|y|} \int_{0}^{1}\left|-y \cdot \nabla K_{k}(x-\theta y)\right| d \theta d x \\
& \leq 2^{-k_{0}+1} \sum_{k \leq k_{0}} \int_{|x| \geq 2|y|} \int_{0}^{1}\left|\nabla K_{k}(x-\theta y)\right| d \theta d x \\
& \leq 2^{-k_{0}+1} \sum_{k \leq k_{0}} \int_{|x| \geq 2|y|} \int_{0}^{1}\left|\nabla K_{k}(x-\theta y)\right|\left(1+2^{k}|x-\theta y|\right)^{1 / 4} d \theta d x \\
& \leq 2^{-k_{0}+1} \sum_{k \leq k_{0}} \int_{0}^{1} \int_{\mathbb{R}^{n}}\left|\nabla K_{k}(x-\theta y)\right|\left(1+2^{k}|x-\theta y|\right)^{1 / 4} d x d \theta \\
& \leq 2^{-k_{0}+1} \sum_{k \leq k_{0}} 2^{k} C \leq C^{\prime},
\end{aligned}
$$

using (4.1). Thus,

$$
\sup _{y \neq 0} \int_{|x| \geq 2|y|}|K(x-y)-K(x)| d x \leq C^{\prime}<\infty
$$

holds, and by Theorem 1.5, T, which is the operator given by convolution against $K=\check{m}$, is strong type $(p, p)$ for $1<p<\infty$; that is, $m \in M_{p}^{p}$ for any $1<p<\infty$.

Example 4.6. Let $m \in C^{\infty}$ on $\mathbb{R}^{n} \backslash\{0\}$ such that there exists $\tau>0$ with

$$
m(\lambda \xi)=\lambda^{i \tau} m(\xi)
$$

for any $\lambda>0$. We prove that $m$ is an $M_{p}^{p}$ multiplier for any $1<p<\infty$. To begin with, notice that

$$
|m(\lambda \xi)|=\left|\lambda^{i \tau}\right||m(\xi)|=|m(\xi)| .
$$

Also, Since $m$ is smooth, it is bounded in the unit sphere $S^{n-1}$ by some constant $C>0$. Thus, for any $\xi \in \mathbb{R}^{n} \backslash\{0\}$, we take $\lambda=|\xi|^{-1}$, and we conclude that

$$
|m(\xi)|=|m(\lambda \xi)| \leq C ;
$$

that is, $m$ is bounded on $\mathbb{R}^{n} \backslash\{0\}$. Now, we take derivatives in (4.3), so for $\alpha \in \mathbb{N}^{n}$,

$$
\lambda^{|\alpha|} D^{\alpha} m(\lambda \xi)=\lambda^{i \tau} D^{\alpha} m(\xi),
$$

and taking again $\lambda=|\xi|^{-1}$, we obtain that

$$
\int_{R \leq|\xi| \leq 2 R}\left|D^{\alpha} m(\xi)\right|^{2} d \xi \leq \int_{R \leq|\xi| \leq 2 R} \frac{C_{\alpha}^{2}}{|\xi|^{2|\alpha|}} d \xi=C_{\alpha}^{\prime} \int_{R \leq r \leq 2 R} r^{n-1-2|\alpha|} d r .
$$

The last integral must be examined for each case (namely, $n \neq 2|\alpha|$ and $n=2|\alpha|$ ), but the conclusion is always the same: we can find some constant $C^{\prime}>0$ so that

$$
\int_{R \leq r \leq 2 R} r^{n-1-2|\alpha|} d r \leq C^{\prime} R^{n-2|\alpha|} .
$$

Thus, Mihlin-Hörmander's theorem applies, and so $m \in M_{p}^{p}$ for any $1<p<\infty$. 



\section{Chapter 5}

\section{The ball multiplier}

We have seen already at some points that the boundedness of the Hilbert transform implies the boundedness of a whole set of operators given by multipliers of the form $\chi_{Q}$, where $Q$ is a square in $\mathbb{R}^{n}$, for any $1<p<\infty$. It is natural, then, to ask if the same happens with the so called ball multiplier,

$$
m(\xi)=\chi_{B}(\xi), \forall \xi \in \mathbb{R}^{n}
$$

where $B$ is the Euclidean ball of radius 1 centred at the origin, for which we consider the ball multiplier operator,

$$
T_{B} f=\left(\widehat{f} \chi_{B}\right)^{\vee}
$$

Of course, since $\chi_{B}$ is bounded, it is an $M_{2}^{2}$ multiplier, so the question is whether $\chi_{B}$ is also an $M_{p}^{p}$ for other values of $p$. To begin with, one can see that $T_{B}$ cannot be bounded outside a critical range that depends on the dimension of the Euclidean space (see [7]), which is

$$
\frac{2 n}{n+1}<p<\frac{2 n}{n-1}, n \geq 2
$$

For a long time, it was believed that, nevertheless, $T_{B}$ was bounded in that interval. This was known as the ball conjecture. At the beginning of the 1970's, though, Fefferman proved that, in reality, the ball conjecture is false, and that $T_{B}$ is only bounded from $L^{2}$ to itself. We review here his proof of this fact, which can be found in its original paper [3] and in [6, Section 5.1]. The proof not only uses part of the multiplier's theory exposed in the previous chapters, but also more geometric elements such as the Perron's tree construction for the Kakeya's problem. The scheme is the following: in the first section, we will see Perron's tree construction, and use it to obtain sets with particular properties regarding their area; we will later on use the ball conjecture to give a result on boundedness of certain multipliers of half-planes; finally, we will see how the initial Perron's tree construction actually allows us to build a counterexample for the former statement, so that the assumption that the ball conjecture holds must be false. 


\subsection{Perron's tree}

We start with a triangle $A B C$ of height $h_{0}$ and base $b$ as in figure 5.1. At height $h_{1}>h_{0}$, we take a horizontal line, and we extend the segments $A B$ and $C B$ until they reach this former horizontal line, cutting it at points $C^{\prime}$ and $A^{\prime}$, respectively. Finally, we take $M$ to be the middle point of the segment $A C$, and we consider the segments $A^{\prime} M$ and $C^{\prime} M$.

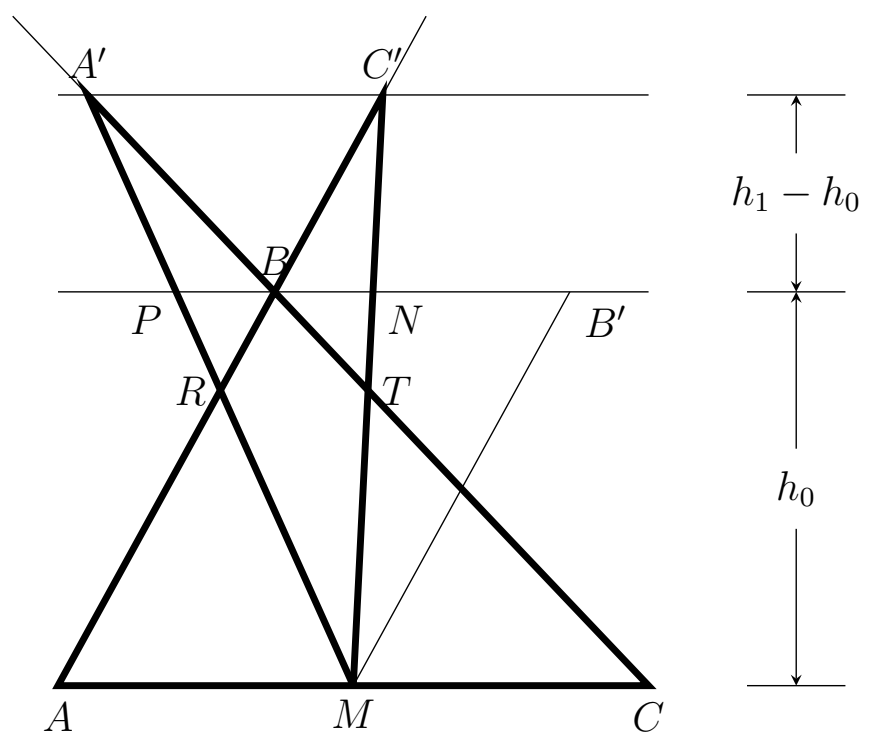

Figure 5.1: Sprouting of triangle $A B C$.

This is the first stage of Perron's tree. We call the triangles $A C^{\prime} M$ and $C A^{\prime} M$ the sprouts of $A B C$ at height $h_{1}$. The triangles $R A^{\prime} B$ and $T C^{\prime} B$ are called the branches of the sprouts. We shall now compute the area of each branch.

Proposition 5.1. With the previous notation, the area of each of the branches of an sprouted triangle is ${ }^{1}$

$$
A_{1}=\frac{b}{2} \frac{\left(h_{1}-h_{0}\right)^{2}}{2 h_{1}-h_{0}} .
$$

Proof. As in figure 5.1, consider a horizontal line at height $h_{0}$, and let $P$ and $N$ be the intersections of such line with the segments $A^{\prime} M$ and $C^{\prime} M$. We will do the computations only for the branch $R A^{\prime} B$, since the same argument is valid for the other one.

We split the triangle $R A^{\prime} B$ in the two triangles $P A^{\prime} B$ and $R P B$, and we compute the area of each of them. To do so, notice that the triangle $P A^{\prime} B$ is similar to the triangle $M A^{\prime} C$, so by Thales' theorem,

$$
\frac{h_{1}-h_{0}}{h_{1}}=\frac{|P B|}{b / 2} .
$$

\footnotetext{
${ }^{1}$ Notice that we are not doing any assumption on the regularity of the triangle $A B C$.
} 
Now, take a parallel segment from $M$ to the segment $A B$, obtaining the point $B^{\prime}$ at height $h_{0}$. The triangle $R P B$ is similar to the triangle $M P B^{\prime}$, and we also have that $\left|P B^{\prime}\right|=|P B|+b / 2$, so if $h_{R P B}$ represents the height of the triangle $R P B$, we have again by Thales' theorem that

$$
\frac{h_{R P B}}{h_{0}}=\frac{|P B|}{|P B|+b / 2} .
$$

With this, we conclude that the area of the triangle $R P B$ is

$$
A_{R P B}=\frac{|P B| h_{R P B}}{2}=\frac{h_{0}|P B|^{2}}{2|P B|+b}=\frac{h_{0}\left(\frac{b\left(h_{1}-h_{0}\right)}{2 h_{1}}\right)^{2}}{\frac{b\left(h_{1}-h_{0}\right)}{h_{1}}+b}=\frac{h_{0} b\left(h_{1}-h_{0}\right)^{2}}{4\left(2 h_{1}-h_{0}\right) h_{1}},
$$

while the area of the triangle $P A^{\prime} B$ is

$$
A_{P A^{\prime} B}=\frac{|P B|\left(h_{1}-h_{0}\right)}{2}=\frac{b\left(h_{1}-h_{0}\right)^{2}}{4 h_{1}}
$$

and so

$$
\begin{aligned}
A_{1} & =\frac{h_{0} b\left(h_{1}-h_{0}\right)^{2}}{4\left(2 h_{1}-h_{0}\right) h_{1}}+\frac{b\left(h_{1}-h_{0}\right)^{2}}{4 h_{1}} \\
& =\frac{h_{0} b\left(h_{1}-h_{0}\right)^{2}+b\left(h_{1}-h_{0}\right)^{2}\left(2 h_{1}-h_{0}\right)}{4\left(2 h_{1}-h_{0}\right) h_{1}} \\
& =\frac{b\left(h_{1}-h_{0}\right)^{2}}{2\left(2 h_{1}-h_{0}\right)} .
\end{aligned}
$$

Given the sprouts of the previous triangle $A B C$, we can now repeat the same process with such sprouts and a new height $h_{2}>h_{1}$ (figure 5.2). By the previous proposition, the area of these new branches will be

$$
A_{2}=\frac{b / 2}{2} \frac{\left(h_{2}-h_{1}\right)^{2}}{2 h_{2}-h_{1}}=\frac{b}{4} \frac{\left(h_{2}-h_{1}\right)^{2}}{2 h_{2}-h_{1}},
$$

for each of the sprouts has height $h_{1}$ and base $b / 2$. We shall call this the second stage of Perron's tree. In general, given the $(N-1)$-th stage of Perron's tree at height $h_{N-1}$, and a new height $h_{N}>h_{N-1}$, we can take all $2^{N-1}$ sprouts and apply the sprouting process to each of them, obtaining a total amount of $2^{N}$ new sprouts, the branches of which have area equal to

$$
A_{N}=\frac{b}{2^{N}} \frac{\left(h_{N}-h_{N-1}\right)^{2}}{2 h_{N}-h_{N-1}} .
$$



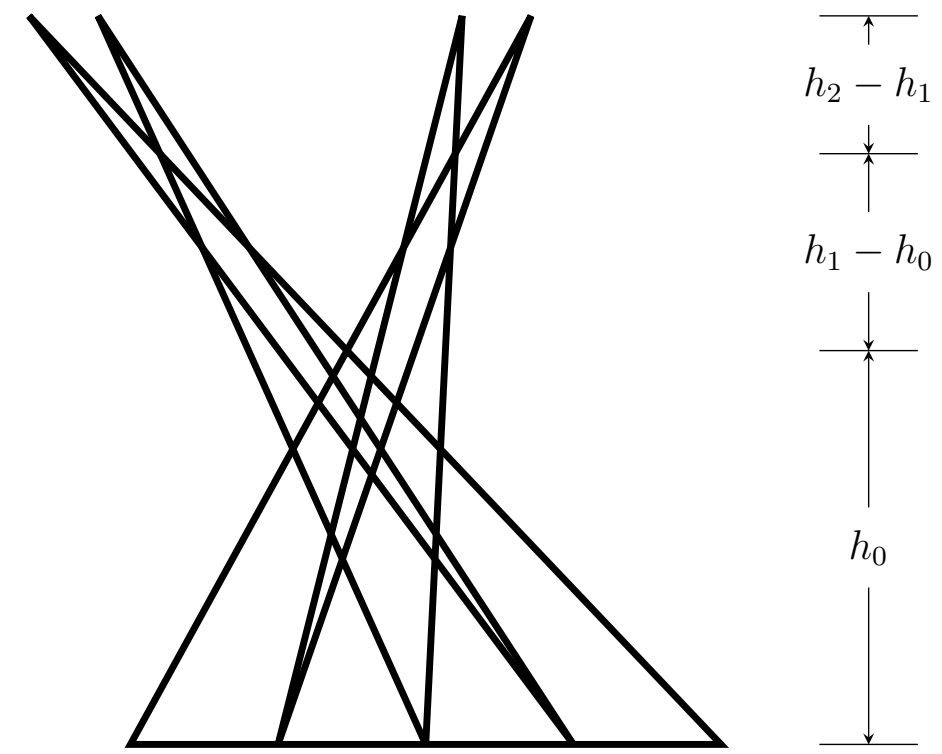

Figure 5.2: Sprouting of the sprouts of the triangle $A B C$.

In the particular case when

$$
h_{k}=h_{0} \sum_{l=0}^{k} \frac{1}{l+1}, \forall k=1, \ldots, N,
$$

and we take our initial triangle $A B C$ to be isosceles with $b=h_{0}$, we have that the total area of the $N$-th stage of Perron's tree will satisfy

$$
\begin{aligned}
A_{N}^{T} & =\frac{h_{0}^{2}}{2}+\sum_{k=1}^{N} 2^{k} A_{k}=\frac{h_{0}^{2}}{2}+{ }^{2} \sum_{k=1}^{N} \frac{\left(h_{k}-h_{k-1}\right)^{2}}{2 h_{k}-h_{k-1}} \\
& =\frac{h_{0}^{2}}{2}+h_{0}^{3} \sum_{k=1}^{N} \frac{1}{(k+1)^{2}} \frac{1}{2 h_{k}-h_{k-1}} \\
& =\frac{h_{0}^{2}}{2}+h_{0}^{3} \sum_{k=1}^{N} \frac{1}{(k+1)^{2}} \frac{1}{h_{k}+h_{0} /(k+1)} \\
& \leq \frac{h_{0}^{2}}{2}+h_{0}^{3} \sum_{k=1}^{N} \frac{1}{(k+1)^{2}} \frac{1}{h_{0}+h_{0} /(k+1)} \\
& \leq \frac{h_{0}^{2}}{2}+h_{0}^{2} \sum_{k=1}^{N} \frac{1}{(k+1)^{2}} \leq \frac{h_{0}^{2}}{2}+h_{0}^{2} \sum_{k=2}^{\infty} \frac{1}{k^{2}} \\
& =h_{0}^{2}\left(\frac{1}{2}+\frac{\pi^{2}}{6}-1\right) \leq \frac{3}{2} h_{0}^{2} .
\end{aligned}
$$

This fact allows us to construct certain sets with a very particular property. Given a rectangle $R$, let $R^{\prime}$ be the set formed by two copies of $R$ adjacent to $R$ along its shortest sides (figure 5.3). With this, we have the following result. 


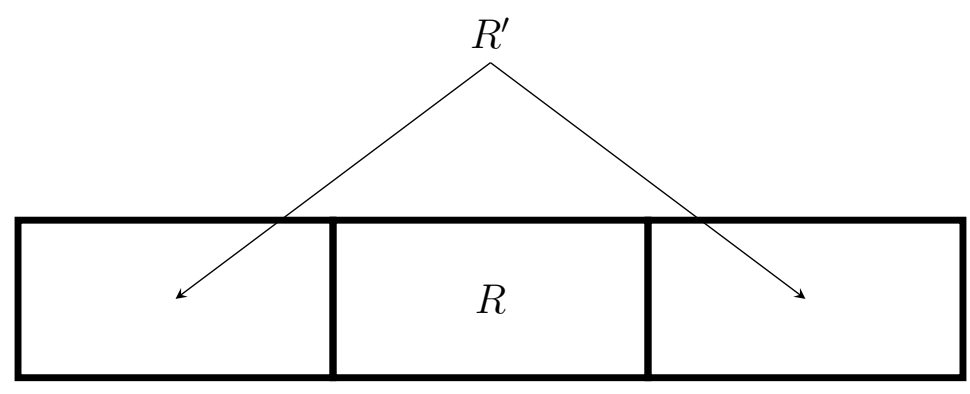

Figure 5.3: $R$ and $R^{\prime}$.

Theorem 5.2. Given $\eta>0$, there exist a set $E$ and a family of disjoint rectangles $\left\{R_{j}\right\}_{j}$ such that

i) $\left|R_{j}^{\prime} \cap E\right| \geq 2\left|R_{j}\right| / 15$,

ii) $|E| \leq \eta \sum_{j}\left|R_{j}\right|$,

Proof. Take $N \geq 1$, and take an isosceles triangle such that its base is the interval $[0,1]$ and with height $h_{0}=1$, and denote by $E_{N}$ its Perron's tree of stage $N$, with heights $h_{k}$ given by (5.1). Because of this choice of heights, it is easily observed that

$$
\log (N+2) \leq h_{N} \leq \log (N+1)+1 .
$$

The tree is made out of $2^{N}$ sprouts $S_{j}$, each of them with base equal to $2^{-N}$. Thus, the area of each sprout is the same, and it is equal to

$$
A_{s}=2^{-(N+1)} \sum_{l=0}^{N} \frac{1}{l+1} .
$$

We denote by $A_{j}$ and $B_{j}$ the left and right vertices of the base of the sprout $j$, $j=1, \ldots, 2^{N}$, and $C_{j}$ the remaining vertex of the sprout at height $h_{N}$. Notice that $A_{1}=A=(0,0), B_{2^{N}}=B=(1,0)$, and $B_{j}=A_{j+1}$, for $j=1, \ldots, 2^{N}-1$. Also, we clearly have that the segments $A C_{1}$ and $B C_{2^{N}}$ have equal length $s_{N}$, and it is maximal among all the lengths of the segments $A_{j} C_{j}$ and $B_{j} C_{j}$. Actually, by Thales' and Pythagoras' theorems, we have that

$$
s_{N}=\sqrt{1^{2}+(1 / 2)^{2}} h_{N}=\frac{\sqrt{5}}{2} h_{N},
$$

and by (5.3), it holds for any segment $A_{j} C_{j}$ and $B_{j} C_{j}$ that

$$
\begin{aligned}
\left|A_{j} C_{j}\right|,\left|B_{j} C_{j}\right| & \leq s_{N}=\frac{\sqrt{5}}{2} h_{N} \leq \frac{\sqrt{5}}{2}(\log (N+1)+1) \leq \frac{3}{2}(\log (N+1)+1) \\
& =\frac{3}{2}\left[\log (N+2)+\log \left(e \frac{N+1}{N+2}\right)\right] \leq \frac{3}{2}(\log (N+2)+\log (N+1)) \\
& \leq 3 \log (N+2),
\end{aligned}
$$

because $N \geq 1$, so that $e \leq N+2$. With this, we define $R_{j}$ as in figure 5.4 for each $j=1, \ldots, 2^{N}$, where $l_{N}=3 \log (N+2)$. 


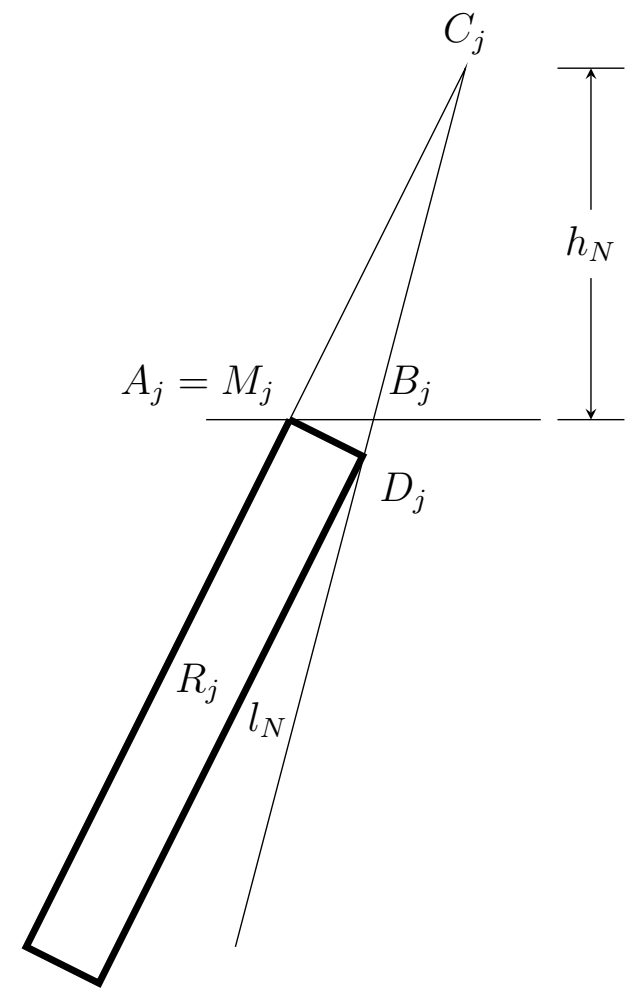

Figure 5.4: Rectangle $R_{j}$ associated to the sprout $A_{j} B_{j} C_{j}$.

It is clear that these rectangles are pairwise disjoint, for each of them is contained in regions that are pairwise disjoint too. Let us estimate the area of $R_{j}$. By the sine theorem,

$$
\begin{aligned}
\left|R_{j}\right| & =3 \log (N+2)\left|M_{j} D_{j}\right|=\frac{3 \log (N+2)}{2^{N}} \frac{\sin \left(\widehat{A_{j} B_{j} D_{j}}\right)}{\sin \left(\widehat{A_{j} D_{j} B_{j}}\right)} \\
& \leq \frac{3 \log (N+2)}{2^{n}} \frac{1}{\sin \left(\widehat{A_{j} D_{j} B_{j}}\right)}=\frac{3 \log (N+2)}{2^{N}} \frac{1}{\cos \left(\widehat{A_{j} C_{j} B_{j}}\right)}
\end{aligned}
$$

but by the cosine theorem, denoting as $C_{j}^{\prime}$ the projection of $C_{j}$ onto the horizontal axis, we have

$$
\begin{aligned}
\cos \left(\widehat{A_{j} C_{j} B_{j}}\right) & =\frac{\left|A_{j} C_{j}\right|^{2}+\left|B_{j} C_{j}\right|^{2}-\left|A_{j} B_{j}\right|^{2}}{2\left|A_{j} C_{j}\right|\left|B_{j} C_{j}\right|} \\
& =\frac{\left|A_{j} C_{j}\right|^{2}-\left|A_{j} C_{j}^{\prime}\right|^{2}+\left|B_{j} C_{j}\right|^{2}-\left|B_{j} C_{j}^{\prime}\right|^{2}+2\left|B_{j} C_{j}^{\prime}\right|\left|A_{j} C_{j}^{\prime}\right|}{2\left|A_{j} C_{j}\right|\left|B_{j} C_{j}\right|} \\
& =\frac{2 h_{N}^{2}+2\left|B_{j} C_{j}^{\prime}\right|\left|A_{j} C_{j}^{\prime}\right|}{2\left|A_{j} C_{j}\right|\left|B_{j} C_{j}\right|} \geq \frac{2 h_{N}^{2}}{2 \frac{5}{4} h_{N}^{2}}=\frac{4}{5}
\end{aligned}
$$

so in the end,

$$
\left|R_{j}\right| \leq \frac{15}{2} \frac{\log (N+2)}{2^{N+1}}
$$


Now, clearly, the set $R_{j}^{\prime}$ will intersect $E_{N}$ with at least the $j$-th sprout, and because of (5.4), (5.3) and (5.5) (in that order), we actually have that

$$
\left|R_{j}^{\prime} \cap E_{N}\right| \geq\left|R_{j}^{\prime} \cap S_{j}\right|=\left|S_{j}\right|=\frac{h_{N}}{2^{N+1}} \geq \frac{\log (N+2)}{2^{N+1}} \geq \frac{2}{15}\left|R_{j}\right|,
$$

which is property i) of the theorem. To prove ii), we simply have, again by the sine theorem, that

$$
\begin{aligned}
\left|R_{j}\right| & =\frac{3 \log (N+2)}{2^{N}} \frac{\sin \left(\widehat{A_{j} B_{j} D_{j}}\right)}{\sin \left(\widehat{A_{j} D_{j} B_{j}}\right)} \geq \frac{3 \log (N+2)}{2^{N}} \sin \left(\widehat{A_{j} B_{j} D_{j}}\right) \\
& \geq \frac{3 \log (N+2)}{2^{N}} \sin \left(\widehat{A_{1} B_{1} D_{1}}\right)=\frac{3 \log (N+2)}{2^{N}} \frac{1}{\frac{\sqrt{5}}{2}} \\
& \geq \frac{\log (N+2)}{2^{N-1}},
\end{aligned}
$$

SO

$$
\sum_{j}\left|R_{j}\right| \geq 2^{N} \frac{\log (N+2)}{2^{N-1}}=2 \log (N+2),
$$

but $\left|E_{N}\right| \leq 3 / 2$ in virtue of (5.2), so given $\eta>0$ we might just assume that $N$ is large enough so that

$$
\frac{3}{2} \leq 2 \eta \log (N+2)
$$

and thus ii) follows, taking $E=E_{N}$.

\subsection{Boundedness on half-planes}

Assume the ball conjecture is true, and that $T_{B}$ is strong type $(p, p)$ for some $p \neq 2$. From this, we are now going to prove a statement that regards boundedness of families of multiplier operators of half-planes. The idea is to use $T_{B}$ to approximate those operators by considering disks of increasing radii, and see how the presumed boundedness of $T_{B}$ affects the boundedness of the family. We start with a technical lemma.

Lemma 5.3. Let $R$ be a rectangle in $\mathbb{R}^{2}$, and let $v$ be a unitary vector parallel to the longest sides of $R$. Let

$$
\mathcal{H}=\left\{x \in \mathbb{R}^{2}: x \cdot v \geq 0\right\},
$$

and let

$$
T_{\mathcal{H}} f=\left(\widehat{f} \chi_{\mathcal{H}}\right)^{\vee}
$$

be its associated multiplier operator. Then,

$$
\left|T_{\mathcal{H}} \chi_{R}\right| \geq \frac{1}{10} \chi_{R^{\prime}}
$$

where $R^{\prime}$ is as in figure 5.3 . 
Proof. Without loss of generality, we might assume that $R$ is centred at the origin, for $T_{\mathcal{H}}$ is translation invariant. Moreover, we shall also assume that $v=(0,1)$, so in particular $\mathcal{H}=\mathbb{R} \times[0, \infty)$ and $R=[-a, a] \times[-b, b]$ for some $0<a \leq b<\infty$, because the Fourier transform is rotation invariant.

We need to prove that for $x \in R^{\prime}$, we have

$$
\left|T_{\mathcal{H}} \chi_{R}(x)\right| \geq \frac{1}{10}
$$

To do so, take $x=\left(x_{1}, x_{2}\right)$ and $\xi=\left(\xi_{1}, \xi_{2}\right)$, and we start writing

$$
\chi_{\mathcal{H}}(\xi) \widehat{\chi_{R}}(\xi)=\chi_{[0, \infty)}\left(\xi_{2}\right)\left(\chi_{[-a, a]}\left(\xi_{1}\right) \chi_{[-b, b]}\left(\xi_{2}\right)\right)^{\wedge}=\widehat{\chi_{[-a . a]}}\left(\xi_{1}\right) \widehat{\chi_{[-b, b]}}\left(\xi_{2}\right) \chi_{[0, \infty)}\left(\xi_{2}\right),
$$

and because $\chi_{[0, \infty)}$ can be written in terms of the sign function as

$$
\chi_{[0, \infty)}\left(\xi_{2}\right)=\frac{1+\operatorname{sgn}\left(\xi_{2}\right)}{2}=\frac{1+i\left(-i \operatorname{sgn}\left(\xi_{2}\right)\right)}{2},
$$

we have

$$
T_{\mathcal{H}} \chi_{R}(x)=\chi_{[-a, a]}\left(x_{1}\right)\left(\widehat{\chi_{[-b, b]}} \chi_{[0, \infty)}\right)^{\vee}\left(x_{2}\right)=\chi_{[-a, a]}\left(x_{1}\right) \frac{I d+i H}{2} \chi_{[-b, b]}\left(x_{2}\right),
$$

where $I d$ is the identity operator, and $H$ is the Hilbert transform. In particular, for $x \in R^{\prime}$, it holds that $\left|x_{1}\right| \leq a$ and $b<\left|x_{2}\right|<3 b$, so we actually have

$$
T_{\mathcal{H}} \chi_{R}(x)=\frac{i}{2} H \chi_{[-b, b]}\left(x_{2}\right),
$$

and

$$
H \chi_{[-b, b]}\left(x_{2}\right)=\text { p.v. } \frac{1}{\pi} \int_{\mathbb{R}} \frac{\chi_{[-b, b]}(y)}{x_{2}-y} d y=\frac{1}{\pi} \int_{-b}^{b} \frac{1}{x_{2}-y} d y=\frac{1}{\pi} \log \left|\frac{x_{2}+b}{x_{2}-b}\right|,
$$

which yields

$$
\left|T_{\mathcal{H}} \chi_{R}(x)\right|=\frac{1}{2 \pi}|\log | \frac{x_{2}+b}{x_{2}-b}|| .
$$

Finally, notice that when $b<x_{2}<3 b$, we have

$$
\left|\frac{x_{2}+b}{x_{2}-b}\right|-2=\frac{x_{2}+b}{x_{2}-b}-2=\frac{3 b-x_{2}}{x_{2}-b}>0,
$$

so that

$$
|\log | \frac{x_{2}+b}{x_{2}-b}||>\log 2,
$$

while for $-3 b<x_{2}<-b$,

$$
\left|\frac{x_{2}-b}{x_{2}+b}\right|-2=\frac{b-x_{2}}{-b-x_{2}}-2=\frac{3 b+x_{2}}{-b-x_{2}}>0,
$$


meaning that

$$
|\log | \frac{x_{2}+b}{x_{2}-b}||=|\log | \frac{x_{2}-b}{x_{2}+b}||>\log 2 .
$$

Hence, in the end, we conclude

$$
\left|T_{\mathcal{H}} \chi_{R}(x)\right|>\frac{\log 2}{2 \pi}>\frac{1}{10}
$$

as we wanted.

Theorem 5.4. Let $\left(v_{j}\right)_{j}$ be a sequence of unit vectors in $\mathbb{R}^{2}$, and let

$$
\mathcal{H}_{j}=\left\{x \in \mathbb{R}^{2}: x \cdot v_{j} \geq 0\right\}
$$

be the half-plane defined by the vector $v_{j}$. Also, let $\chi_{j}=\chi_{\mathcal{H}_{j}}$, and

$$
T_{j} f=\left(\widehat{f} \chi_{j}\right)^{\vee}
$$

Finally, assume that the ball multiplier operator,

$$
T_{B} f=\left(\widehat{f} \chi_{B}\right)^{\vee},
$$

is bounded for some $1<p<\infty$. Then,

$$
\left\|\left(\sum_{j}\left|T_{j} f_{j}\right|^{2}\right)^{1 / 2}\right\|_{p} \lesssim\left\|\left(\sum_{j}\left|f_{j}\right|^{2}\right)^{1 / 2}\right\|_{p},
$$

for any sequence of functions $\left(f_{j}\right) \subset L^{p}$.

Proof. We shall do the proof for Schwarz functions, as the general statement would follow by density. For each $j$, we consider the family of operators $\left(T_{j}^{r}\right)_{r}$ defined as

$$
T_{j}^{r} f=\left(\widehat{f} \chi_{B\left(r v_{j}, r\right)}\right)^{\vee} .
$$

Notice that, as $r \rightarrow \infty$, we have that $\chi_{B\left(r v_{j}, r\right)} \rightarrow \chi_{j}$ pointwise, so we expect as well some sort of convergence of the family $\left(T_{j}^{r}\right)_{r}$ towards $T_{j}$. Indeed, take $x \in \mathbb{R}^{2}$. We have that

$$
\begin{aligned}
\left|T_{j}^{r} f(x)-T_{j} f(x)\right| & \leq \int_{\mathbb{R}^{2}}\left|\widehat{f}(\xi) \chi_{B\left(r v_{j}, r\right)}(\xi)-\widehat{f}(\xi) \chi_{j}(\xi)\right| d \xi \\
& =\int_{\mathbb{R}^{2}}\left|\chi_{j}(\xi)-\chi_{B\left(r v_{j}, r\right)}(\xi)\right||\widehat{f}(\xi)| d \xi
\end{aligned}
$$

but all the functions $|\widehat{f}|\left|\chi_{j}-\chi_{B\left(r v_{j}, r\right)}\right|$ are integrable functions that converge pointwise to zero when $r \rightarrow \infty$ and that are bounded from above by $\left|\widehat{f} \chi_{j}\right|$, so by the dominated convergence theorem we conclude

$$
\lim _{r \rightarrow \infty}\left|T_{j}^{r} f(x)-T_{j} f(x)\right| \leq \int_{\mathbb{R}^{2}} \lim _{r \rightarrow \infty}|\widehat{f}(\xi)|\left|\chi_{j}(\xi)-\chi_{B\left(r v_{j}, r\right)}(\xi)\right| d \xi=0,
$$


meaning that $T_{j}^{r} f$ converges pointwise to $T_{j} f$ when $r \rightarrow \infty$. In particular, by Fatou's lemma [1, page 11], we have that

$$
\left\|\left(\sum_{j}\left|T_{j} f_{j}\right|^{2}\right)^{1 / 2}\right\|_{p} \leq \liminf _{r \rightarrow \infty}\left\|\left(\sum_{j}\left|T_{j}^{r} f_{j}\right|^{2}\right)^{1 / 2}\right\|_{p},
$$

so it suffices to see that

$$
\left\|\left(\sum_{j}\left|T_{j}^{r} f_{j}\right|^{2}\right)^{1 / 2}\right\|_{p} \leq C\left\|\left(\sum_{j}\left|f_{j}\right|^{2}\right)^{1 / 2}\right\|_{p},
$$

for any $r>0$, where $C$ is a constant independent of $r$. To do so, first write

$$
\begin{aligned}
T_{j}^{r} f_{j}(x) & =\left(\widehat{f}_{j} \chi_{B\left(r v_{j}, r\right)}\right)^{\vee}(x)=\left(\tau_{-r v_{j}}\left(\left(\tau_{r v_{j}} \widehat{f}_{j}\right) \chi_{B(0, r)}\right)\right)^{\vee}(x) \\
& =e^{2 \pi i r v_{j} \cdot x}\left(\left(\tau_{r v_{j}} \widehat{f}_{j}\right) \chi_{B(0, r)}\right)^{\vee}(x) \\
& =e^{2 \pi i r v_{j} \cdot x}\left(\left(e^{-2 \pi i r v_{j} \cdot} f_{j}\right)^{\wedge} \chi_{B(0, r)}\right)^{\vee}(x) \\
& :=e^{2 \pi i r v_{j} \cdot x} T^{r}\left(e^{-2 \pi i r v_{j} \cdot} f_{j}\right)(x),
\end{aligned}
$$

so that, if $g_{j}=e^{-2 \pi i r v_{j}} \cdot f_{j}$, then

$$
\left\|\left(\sum_{j}\left|T_{j}^{r} f_{j}\right|^{2}\right)^{1 / 2}\right\|_{p}=\left\|\left(\sum_{j}\left|T^{r} g_{j}\right|^{2}\right)^{1 / 2}\right\|_{p} .
$$

Now, the operator $T^{r}$ has the same norm as the ball operator $T_{B}$, for its multiplier is simply a dilation of the former (Proposition 2.18). Therefore, $T^{r}$ is strong type $(p, p)$ by hypothesis, and applying Theorem 1.9, we conclude that

$$
\begin{aligned}
\left\|\left(\sum_{j}\left|T_{j}^{r} f_{j}\right|^{2}\right)^{1 / 2}\right\|_{p} & \leq C\left(p, M_{p}^{p}\left(\chi_{B}\right)\right)\left\|\left(\sum_{j}\left|g_{j}\right|^{2}\right)^{1 / 2}\right\|_{p} \\
& =C\left(p, M_{p}^{p}\left(\chi_{B}\right)\right)\left\|\left(\sum_{j}\left|f_{j}\right|^{2}\right)^{1 / 2}\right\|_{p},
\end{aligned}
$$

as we wanted.

Remark 5.5. It is important to observe that this proof cannot be replicated, for instance, using squares instead of balls to approximate the half-planes $\mathcal{H}_{j}$. Indeed, suppose we tried to. We could think of using, for each half-plane $\mathcal{H}_{j}$, a sequence of squares $Q_{j}\left(r v_{j}, r\right)$ such that one side of the square is on $\partial \mathcal{H}_{j}$ (thus, in particular, $\left.Q_{j}\left(r v_{j}, r\right) \subset \mathcal{H}_{j}\right)$. With this, we would still have that $\chi_{Q_{j}\left(r v_{j}, r\right)} \rightarrow \chi_{j}$ when $r \rightarrow \infty$ pointwise, and so we could conclude again that $T_{j}^{r} f$ converges pointwise to $T_{j} f$ when $r \rightarrow \infty$. Applying again Fatou's lemma, we could reduce again the problem to see that $\vec{T}=\left(T_{j}^{r}\right)_{j}$ is a bounded operator on $L^{p}\left(\mathbb{R}^{n}, \ell^{2}\right)$. But now, we would have that

$$
\begin{aligned}
T_{j}^{r} f_{j}(x) & =\left(\widehat{f}_{j} \chi_{Q_{j}\left(r v_{j}, r\right)}\right)^{\vee}(x)=\left(\tau_{-r v_{j}}\left(\left(\tau_{r v_{j}} \widehat{f}_{j}\right) \chi_{Q_{j}(0, r)}\right)\right)^{\vee}(x) \\
& =e^{2 \pi i r v_{j} \cdot x}\left(\left(\tau_{r v_{j}} \widehat{f}_{j}\right) \chi_{Q_{j}(0, r)}\right)^{\vee}(x) \\
& =e^{2 \pi i r v_{j} \cdot x}\left(\left(e^{-2 \pi i r v_{j} \cdot} f_{j}\right)^{\wedge} \chi_{Q_{j}(0, r)}\right)^{\vee}(x),
\end{aligned}
$$


and though the squares $Q_{j}(0, r)$ have the same center and radius, they are rotated, in general, with respect to the square $\left\{x \in \mathbb{R}^{2}:\|x\|_{\infty} \leq r\right\}$. This means that each multiplier $\chi_{Q_{j}(0, r)}$ is different, so we cannot proceed using Theorem 1.9 as before.

\subsection{The answer to the ball multiplier problem}

We are now ready to disprove the ball multiplier problem; that is, to prove that $T_{B}$ is not strong type $(p, p)$ for any $1<p<\infty$ different from $p=2$.

Theorem 5.6. The ball conjecture is false: $T_{B}$ is only strong type $(p, p)$ for $p=2$.

Proof. By de Leeuw's theorem (Theorem 2.21), it suffices to do the case $n=2$. Moreover, by symmetry (Theorem 2.15), we might just assume that $p>2$. Take any $\eta>0$, and let $E$ and $\left\{R_{j}\right\}_{j}$ be given by Theorem 5.2. For each $j$, take $v_{j}$ an unitary vector parallel to the long side of $R_{j}$, and let $\mathcal{H}_{j}$ be the half-plane defined by $v_{j}$, and $T_{j}$ its associated multiplier operator (as in Theorem 5.4). Finally, let $f_{j}=\chi_{R_{j}}$. By the monotone convergence theorem, one has

$$
\int_{E} \sum_{j}\left|T_{j} f_{j}(x)\right|^{2} d x=\sum_{j} \int_{E}\left|T_{j} f_{j}(x)\right|^{2} d x .
$$

By Lemma 5.3, we get

$$
\sum_{j} \int_{E}\left|T_{j} f_{j}(x)\right|^{2} d x \geq \sum_{j} \int_{E} \frac{1}{10^{2}}\left|\chi_{R_{j}^{\prime}}(x)\right|^{2} d x=\frac{1}{100} \sum_{j}\left|R_{j}^{\prime} \cap E\right|,
$$

and by Theorem 5.2,

$$
\frac{1}{100} \sum_{j}\left|R_{j}^{\prime} \cap E\right| \geq \frac{1}{750} \sum_{j}\left|R_{j}\right| .
$$

On the other hand, since $p>2$, we can apply Hölder's inequality with $p / 2$ and $(p / 2)^{\prime}=p /(p-2)$, and Theorem 5.4 to get

$$
\begin{aligned}
\int_{E} \sum_{j}\left|T_{j} f_{j}(x)\right|^{2} d x & \leq|E|^{(p-2) / p}\left\|\left(\sum_{j}\left|T_{j} f_{j}\right|^{2}\right)^{1 / 2}\right\|_{p}^{2} \\
& \lesssim|E|^{(p-2) / p}\left\|\left(\sum_{j}\left|f_{j}\right|^{2}\right)^{1 / 2}\right\|_{p}^{2} \\
& =|E|^{(p-2) / p}\left\|\left(\sum_{j}\left|\chi_{R_{j}}\right|^{2}\right)^{1 / 2}\right\|_{p}^{2}
\end{aligned}
$$

but because the rectangles $R_{j}$ are disjoint,

$$
|E|^{(p-2) / p}\left\|\left(\sum_{j}\left|\chi_{R_{j}}\right|^{2}\right)^{1 / 2}\right\|_{p}^{2}=|E|^{(p-2) / p}\left(\sum_{j}\left|R_{j}\right|\right)^{2 / p}
$$


and using again Theorem 5.2, we conclude

$$
|E|^{(p-2) / p}\left(\sum_{j}\left|R_{j}\right|\right)^{2 / p} \leq \eta \sum_{j}\left|R_{j}\right| .
$$

All in all, we have obtained that

$$
\begin{aligned}
\frac{1}{750} \sum_{j}\left|R_{j}\right| & \lesssim \eta \sum_{j}\left|R_{j}\right| \\
\frac{1}{750} & \lesssim \eta,
\end{aligned}
$$

but $\eta$ was arbitrary, which is therefore a contradiction. The error can only be in Theorem 5.4, which assumes that $T_{B}$ is strong type $(p, p)$. Thus, the former theorem is disproved, and the ball conjecture is false.

It is interesting to contrast this fact with what happens with the multiplier operators of polygons. Let $P$ be a convex polygon of $k$ sides $a_{1}, \ldots, a_{k}$. Notice that we can write the characteristic function of the polygon as

$$
\chi_{P}=\chi_{\mathcal{H}_{1}} \cdots \chi_{\mathcal{H}_{k}},
$$

where $\mathcal{H}_{i}$ is the half-plane of $\mathbb{R}^{2}$ such that the side $a_{i}$ is contained in $\partial \mathcal{H}_{i}$ and $P \subset \mathcal{H}_{i}$ (see figure 5.5). But each function $\chi_{\mathcal{H}_{i}}$ is an $M_{p}^{p}$ multiplier, for $1<p<\infty$ (use Example 2.20 and apply some rotation). Thus, by Proposition 2.19, $\chi_{P}$ is also an $M_{p}^{p}$ multiplier. Of course, the same argument works for proving that if $P$ is a convex polyhedra in $\mathbb{R}^{n}$, then $\chi_{P}$ is also an $M_{p}^{p}$ multiplier for $1<p<\infty$. This is counter-intuitive, because geometrically, a disc is nothing more but a limit of polygons inscribed on the disc with an increasing number of sides, and still, the boundedness of their associated multiplier operators is lost completely when passing to the limit.

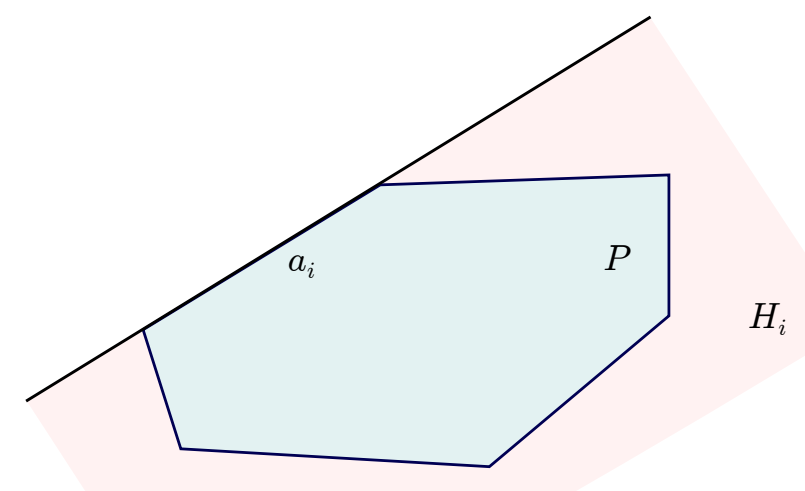

Figure 5.5: Polygon $P$ and half-plane $\mathcal{H}_{i}$. 


\section{Bibliography}

[1] J. Cerdà, Linear Functional Analysis, American Mathematical Society, Real Sociedad Matemática Española, 2010.

[2] J. Duoandikoetxea, Fourier Analysis, American Mathematical Society, 2000.

[3] C. Fefferman, The multiplier problem for the ball, Ann. of Math., 94 no. 2 (1971), $330-336$.

[4] G.B. Folland, Real Analysis: Modern Techniques and Their Applications, Wiley, 2007.

[5] L. Grafakos, Classical Fourier Analysis, Springer-Verlag, New York, 2014.

[6] L. Grafakos, Modern Fourier Analysis, Springer-Verlag, New York, 2014.

[7] C. Herz, On the mean inversion of Fourier and Hankel transforms, Proc. Nat. Acad. Sci. U.S.A. 40 (1954), 996 - 999.

[8] L. Hörmander, Estimates for translation invariant operators in $L^{p}$ spaces, Acta Math. 104 no. 1 - 2 (1960), 93 - 140.

[9] M. Jodeit, A note on Fourier multipliers, Proc. Amer. Math. Soc. 27 no. 2 (1971), $423-424$.

[10] H.L. Royden and P.M. Fitzpatrick, Real Analysis, Prentice Hall, 2010. 



\section{Index}

Ball conjecture, 67

Ball multiplier, 67

Bounded operator, 3

Branch, 68

Conjugate exponent, 4

Convolution, 7

Dual space, 4

Dyadic set, 44, 46

Fourier transform, 8

Functional, 4

Hilbert transform, 14

Inverse Fourier transform, 11

Lebesgue space, 3

Littlewood-Paley operator, 37, 45-47
Multiplier, 18

Multiplier operator, 28

N-th stage of Perron's tree, 69

Perron's tree, 68

Schwarz space, 10

Sobolev space, 12

Sprout, 68

Strong type, 3

Tempered distribution, 11

Translation, 13

Translation invariant operator, 13

Weak $L^{p}$ space, 4

Weak type, 4 